\title{
Remarkable Cryptic Diversity of Paratylenchus spp. (Nematoda: Tylenchulidae) in Spain ${ }^{\dagger}$
}

\author{
Ilenia Clavero-Camacho ${ }^{1}$, Carolina Cantalapiedra-Navarrete ${ }^{1}$, Antonio Archidona-Yuste ${ }^{2,3}$ (D), Pablo Castillo ${ }^{1}$ (D) \\ and Juan Emilio Palomares-Rius 1,*
}

check for updates

Citation: Clavero-Camacho, I.; Cantalapiedra-Navarrete, C.;

Archidona-Yuste, A.; Castillo, P.;

Palomares-Rius, J.E. Remarkable

Cryptic Diversity of Paratylenchus spp. (Nematoda: Tylenchulidae) in Spain.

Animals 2021, 11, 1161. https://

doi.org/10.3390/ani11041161

Academic Editor:

Sergio Álvarez-Ortega

Received: 25 March 2021

Accepted: 15 April 2021

Published: 18 April 2021

Publisher's Note: MDPI stays neutral with regard to jurisdictional claims in published maps and institutional affiliations.

Copyright: (C) 2021 by the authors. Licensee MDPI, Basel, Switzerland. This article is an open access article distributed under the terms and conditions of the Creative Commons Attribution (CC BY) license (https:/ / creativecommons.org/licenses/by/ $4.0 /)$.
1 Campus de Excelencia Internacional Agroalimentario, ceiA3, Instituto de Agricultura Sostenible (IAS), Consejo Superior de Investigaciones Científicas (CSIC), Avenida Menéndez Pidal s/n, 14004 Córdoba, Spain; iclavero@ias.csic.es (I.C.-C.); ccantalapiedra@ias.csic.es (C.C.-N.); p.castillo@csic.es (P.C.)

2 Centro Alameda del Obispo, Andalusian Institute of Agricultural and Fisheries Research and Training (IFAPA), 14004 Córdoba, Spain; antonio.archidona-yuste@ufz.de

3 Helmholtz Centre for Environmental Research-FZ, Department of Ecological Modelling, Permoserstrasse 15, 04318 Leipzig, Germany

* Correspondence: palomaresje@ias.csic.es

+ The ZooBank Life Science Identifier (LSID) for this publication is as follows: http: / / zoobank.org/References/06a10c26-9766-4b32-9346-a3b66dff8928 (accessed on 27 March 2021).

Simple Summary: Paratylenchus spp. are vermiform organisms distributed throughout the world that can parasitize a wide variety of cultivated and wild plants. Some species are considered pathogenic in crops; therefore, correct identification is essential to design management strategies. However, the conserved morphology, similarity of morphometric characters and other factors as co-occurrence of more than one species of Paratylenchus in the same soil sample hinder identification to species level. In consequence, this identification should be carried out jointly with morphological, morphometrical and molecular data. The present research aims to provide morphological and molecular characterization of some Paratylenchus species found in Spain and the description of several new species.

Abstract: In previous studies, fifteen species of Paratylenchus, commonly known as pin nematodes, have been reported in Spain. These plant-parasitic nematodes are ectoparasites with a wide host range and global distribution. In this research, 27 populations from twelve Paratylenchus species from 18 municipalities in Spain were studied using morphological, morphometrical and molecular data. This integrative taxonomic approach allowed the identification of twelve species, four of them were considered new undescribed species and eight were already known described. The new species described here are $P$. caravaquenus sp. nov., P. indalus sp. nov., P. pedrami sp. nov. and P. zurgenerus sp. nov. As for the already known described species, five were considered as first reports for the country, specifically P. enigmaticus, P. hamatus, P. holdemani, P. israelensis, and P. veruculatus, while P. baldaccii, P. goodeyi and P. tenuicaudatus had already been recorded in Spain. This study provides detail morphological and molecular data, including the D2-D3 expansion segments of 28S rRNA, ITS rRNA, and partial mitochondrial COI regions for the identification of different Paratylenchus species found in Spain. These results confirm the extraordinary cryptic diversity in Spain and with examples of morphostatic speciation within the genus Paratylenchus.

Keywords: cytochrome c oxidase subunit 1; ITS rRNA; D2-D3 of 28S rRNA; molecular; morphology; phylogeny; rRNA; taxonomy

\section{Introduction}

Pin nematodes of the genus Paratylenchus Micoletzky, 1922 [1] are one of the smallest plant-parasitic nematodes; their body length varies from 160 to $600 \mu \mathrm{m}$ [2]. Stylet length is the organ which drives the feeding habit and morphology of adult females. Some species 
have a long stylet $(>40 \mu \mathrm{m})$, become swollen and feeding from deeper layers in the root cortex as sedentary ectoparasites. However, the majority of them feed as migratory ectoparasites on epidermal cells and root hairs [2]. In general, Paratylenchus spp. are parasites of higher plants (herbaceous and woody) with a higher abundance in the rhizosphere of trees and perennials [3]. This is probably due to their lifecycle, species with smaller body size and higher fecundity allow for faster build-up of their populations compared to other plant-parasitic species [4-6]. Additionally, they can survive using dehydration and be easily dispersed by wind [7]. Many species arrest their development in the fourth-stage juvenile (J4) and some species may molt to adult with root diffusates from their host plant; although some molting might occur in spring in the absence of root exudates [8]. Paratylenchus species are widely dispersed in different environments and crops, and world-wide distributed. Pathogenicity has been found only on different crops for a few species such as P. bukowinensis Micoletzky, 1922 in celery (Apium graveolens L.) [8,9]; Paratylenchus dianthus Jenkins \& Taylor, 1956 in carnation (Dianthus caryophyllus L.) [10]; Paratylenchus epacris (Allen \& Jensen, 1950) Goodey, 1963 in black walnut (Juglans nigra L.) [11]; Paratylenchus hamatus Thorne \& Allen, 1950 in fig (Ficus carica L.), pear (Pyrus communis L.) and grapevine (Vitis vinifera L.) [12-14]; Paratylenchus microdorus Andrassy, 1959 in red clover (Trifolium pratense L.) and lettuce (Lactuca sativa L.) [15]; Paratylenchus nanus Cobb, 1923 in garden balsam (Impatiens balsamina L.) [16]; Paratylenchus neoamblycephalus Geraert, 1965 in Myrobalan plum (Prunus cerasifera Ehrh.) [17]; Paratylenchus projectus Jenkins, 1956 in alfalfa (Medicago sativa L.) and sunflower (Helianthus annuus L.) [18,19]; Paratylenchus shenzhenensis Wang, Xie, Li, Xu, Yu \& Wang, 2013 in Anthurium andraeanum Linden ex André [20]; and Paratylenchus enigmaticus Munawar, Yevtushenko, Palomares-Rius \& Castillo, 2021 in lettuce [21,22]. Other species can be also pathogenic in crops, but further studies are necessary to confirm this. This lack of knowledge is aggravated by the difficulty of working with these tiny nematodes [3]. In this sense, the correct taxonomic identification for putative species damaging crops is of vital importance for their practical management in field. Paratylenchus identification to species level is hampered by the largely conserved morphology, overlapping morphometrics, high levels of intra-specific variability and, most importantly, the frequent co-occurrence of more than one pin species in the same soil sample $[23,24]$. An additional difficulty is that many of these species are found in the soil as quiescent juvenile stages (usually, the fourth-stage juvenile). They could remain in this stage until suitable environmental conditions and/or appropriate plant host are available. Furthermore, Fisher [25] found that morphological characters used for species identification might be influenced by environmental and other factors (such as temperature, host, population size, etc.). For these reasons, it is essential to identify Paratylenchus species accurately using integrative taxonomic methods (combination of morphological, morphometrical and molecular data), at least for type populations. Several articles have studied in this genus using this methodology, giving interesting results for their molecular variability and the presence of cryptic species using several populations of the same species [21,23,24,26-29]. After the characterization of type population of each species, barcoding techniques could be used more easily and effectively in the future for the management of these nematodes in field. Recently, several studies, some using molecular data, have questioned the monophyly of Tylenchulidae [2,30-32] and include the genus Gracilacus within Paratylenchus [2,33-36]. We follow this last inclusion of all species of Gracilacus in Paratylenchus as also is showed in recently resolved phylogenies in which the genus Gracilacus was distributed along Paratylenchus clades $[24,28,37]$. Likewise, the monotypic genus Cacopaurus characterized by the obese female body, tubercles on annuli of the female cuticle and sessile parasitism [38] was synonymized by Goodey [39], but it has been accepted by several authors [2,24,40,41] and in the last monograph of the Tylenchulidae by Ghaderi et al. [2].

Fifteen species of Paratylenchus have been reported in Spain from cultivated and wild ecosystems including P. arculatus Luc \& de Guiran, 1962 [42,43], P. baldaccii Raski, 1975 [44], P. ciccaronei Raski, 1975 [45-47], P. goodeyi Oostenbrink, 1953 [48], P. macrodorus Brzeski, 1963 [46], P. microdorus [44-47], P. nanus Cobb, 1923 [46,49], P. peraticus (Raski, 1962) 
Siddiqi \& Goodey, 1964 [48], P. sheri (Raski, 1973) Siddiqi, 1986 [45-47,49], P. similis Khan, Prasad \& Mathur, 1967 [46,49], P. steineri Golden, 1961 [46,48], P. straeleni (De Coninck, 1931) Oostenbrink, 1960 [50], P. teres (Raski, 1976) Siddiqi, 1986 [51], P. tenuicaudatus Wu, 1961 [52] and P. vandenbrandei de Grisse, 1962 [45,47]. However, some studies did not use molecular techniques for their identification and the biodiversity of this group could be underexplored. Therefore, a new assessment of species using molecular barcoding for Spanish populations could be of interests for their unequivocal identification and the reliable estimation of biodiversity. This study tries to understand this biodiversity using an integrative taxonomic approach.

The main objectives of this study were to: (i) conduct identification with a morphological and morphometrical approaches of some Paratylenchus species collected in several nematode surveys in Spain; (ii) provide molecular characterization of several species using ribosomal (D2-D3 expansion segments of 28S rRNA, Internal Transcribed Spacer region (ITS) rRNA) and the mitochondrial region cytochrome c oxidase subunit 1 (COI); and (iii) study phylogenetic relationships within Paratylenchus spp. using the obtained molecular markers.

\section{Materials and Methods}

\subsection{Nematode Sampling and Morphological Identification}

Soil samples were collected mainly from the rhizosphere of woody plants including pine (Pinus halepensis Mill.) and several Prunus spp. (almond, apricot, cherry, nectarine and peach) with different rootstocks, in several localities in Spain (Table 1), using a shovel, and considering the upper 5-40 cm depth of soil. Nematodes were extracted from a $500 \mathrm{~cm}^{3}$ sub-sample of soil by centrifugal flotation [53].

Table 1. Isolates sampled and sequenced for Paratylenchus spp. from several localities in Spain used in this study. Different rootstocks could be used in the cultivated plants.

\begin{tabular}{|c|c|c|c|c|c|c|}
\hline Soil Sample Code & Nematode Species & Locality (Province) & Host Plant & D2-D3 & ITS & COI \\
\hline PI_ARC & $\begin{array}{c}\text { Paratylenchus } \\
\text { caravaquenus sp. nov. }\end{array}$ & Caravaca (Murcia) ${ }^{a}$ & pine & $\begin{array}{l}\text { MW798270- } \\
\text { MW798272 }\end{array}$ & $\begin{array}{l}\text { MW798316- } \\
\text { MW798318 }\end{array}$ & $\begin{array}{l}\text { MW797003- } \\
\text { MW797004 }\end{array}$ \\
\hline PR_104 & $\begin{array}{l}\text { Paratylenchus indalus } \\
\text { sp. nov. }\end{array}$ & $\begin{array}{l}\text { Santa María de Nieva } \\
\text { (Almería) }^{\text {a }}\end{array}$ & almond & $\begin{array}{l}\text { MW798273- } \\
\text { MW798275 }\end{array}$ & $\begin{array}{l}\text { MW798319- } \\
\text { MW798322 }\end{array}$ & MW797005 \\
\hline PR_114 & $\begin{array}{l}\text { Paratylenchus indalus } \\
\text { sp. nov. }\end{array}$ & Urracal (Almería) & almond & $\begin{array}{l}\text { MW798276- } \\
\text { MW798277 }\end{array}$ & $\begin{array}{l}\text { MW798323- } \\
\text { MW798324 }\end{array}$ & MW797006 \\
\hline PR_118 & $\begin{array}{l}\text { Paratylenchus indalus } \\
\text { sp. nov. }\end{array}$ & Serón (Almería) & almond & $\begin{array}{l}\text { MW798278- } \\
\text { MW798280 }\end{array}$ & $\begin{array}{l}\text { MW798325- } \\
\text { MW798326 }\end{array}$ & MW797007 \\
\hline PR_119 & $\begin{array}{l}\text { Paratylenchus indalus } \\
\text { sp. nov. }\end{array}$ & El Hijate (Almería) & almond & $\begin{array}{l}\text { MW798281- } \\
\text { MW798282 }\end{array}$ & $\begin{array}{l}\text { MW798327- } \\
\text { MW798328 }\end{array}$ & MW797008 \\
\hline PR_014 & $\begin{array}{l}\text { Paratylenchus pedrami } \\
\text { sp. nov. }\end{array}$ & Córdoba (Córdoba) ${ }^{a}$ & almond & MW798283 & MW798329 & MW797009 \\
\hline PR_017 & $\begin{array}{l}\text { Paratylenchus pedrami } \\
\text { sp. nov. }\end{array}$ & Córdoba (Córdoba) & almond & $\begin{array}{l}\text { MW798284- } \\
\text { MW798285 }\end{array}$ & MW798330 & - \\
\hline PR_111 & $\begin{array}{c}\text { Paratylenchus zurgenerus } \\
\text { sp. nov. }\end{array}$ & Zurgena (Almería) a & almond & $\begin{array}{l}\text { MW798286- } \\
\text { MW798289 }\end{array}$ & $\begin{array}{l}\text { MW798331- } \\
\text { MW798334 }\end{array}$ & $\begin{array}{l}\text { MW797010- } \\
\text { MW797011 }\end{array}$ \\
\hline PR_152 & $\begin{array}{c}\text { Paratylenchus baldaccii } \\
\text { Raski, } 1975\end{array}$ & Cantillana (Sevilla) & peach & $\begin{array}{l}\text { MW798290- } \\
\text { MW798291 }\end{array}$ & $\begin{array}{l}\text { MW798335- } \\
\text { MW798336 }\end{array}$ & MW797012 \\
\hline PR_193 & $\begin{array}{l}\text { Paratylenchus enigmaticus } \\
\text { Munawar, } 2021\end{array}$ & La Almunia (Zaragoza) & cherry & MW798292 & MW798337 & MW797013 \\
\hline PR_014 & $\begin{array}{l}\text { Paratylenchus goodeyi } \\
\text { (Oostenbrink, 1953) } \\
\text { Raski, } 1962\end{array}$ & Córdoba (Córdoba) & almond & $\begin{array}{l}\text { MW798293- } \\
\text { MW798294 }\end{array}$ & $\begin{array}{l}\text { MW798338- } \\
\text { MW798339 }\end{array}$ & $\begin{array}{l}\text { MW797014- } \\
\text { MW797015 }\end{array}$ \\
\hline PR-044 & $\begin{array}{l}\text { Paratylenchus hamatus } \\
\text { Thorne \& Allen, } 1950\end{array}$ & Gibraleón (Huelva) & peach & MW798295 & MW798340 & MW797016 \\
\hline PR_115 & $\begin{array}{l}\text { Paratylenchus hamatus } \\
\text { Thorne \& Allen, } 1950\end{array}$ & Lúcar (Almería) & almond & MW798296 & MW798341 & - \\
\hline PR_207 & $\begin{array}{l}\text { Paratylenchus hamatus } \\
\text { Thorne \& Allen, } 1950\end{array}$ & Sástago (Zaragoza) & peach & MW798297 & - & MW797017 \\
\hline PR_187 & $\begin{array}{l}\text { Paratylenchus hamatus } \\
\text { Thorne \& Allen, } 1950\end{array}$ & Ariza (Zaragoza) & almond & $\begin{array}{l}\text { MW798298- } \\
\text { MW798299 }\end{array}$ & - & - \\
\hline PR_082 & $\begin{array}{l}\text { Paratylenchus holdemani } \\
\text { Raski, } 1975\end{array}$ & Martos (Jaén) & almond & MW798300 & MW798342 & MW797018 \\
\hline
\end{tabular}


Table 1. Cont.

\begin{tabular}{|c|c|c|c|c|c|c|}
\hline Soil Sample Code & Nematode Species & Locality (Province) & Host Plant & D2-D3 & ITS & COI \\
\hline PR_079 & $\begin{array}{l}\text { Paratylenchus israelensis } \\
\text { (Raski, 1973) Siddiqi, } 1986\end{array}$ & Valenzuela (Córdoba) & almond & $\begin{array}{l}\text { MW798301- } \\
\text { MW798302 }\end{array}$ & $\begin{array}{l}\text { MW798343- } \\
\text { MW798346 }\end{array}$ & - \\
\hline PR_011 & $\begin{array}{l}\text { Paratylenchus israelensis } \\
\text { (Raski, 1973) Siddiqi, } 1986\end{array}$ & Córdoba (Córdoba) & almond & $\begin{array}{l}\text { MW798303- } \\
\text { MW798305 }\end{array}$ & 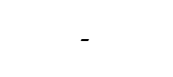 & $\begin{array}{l}\text { MW797019- } \\
\text { MW797020 }\end{array}$ \\
\hline PR_124 & $\begin{array}{c}\text { Paratylenchus tenuicaudatus } \\
\text { Wu, } 1961\end{array}$ & Caravaca (Murcia) & almond & MW798306 & MW798347 & MW797021 \\
\hline PR_129 & $\begin{array}{c}\text { Paratylenchus tenuicaudatus } \\
\text { Wu, } 1961\end{array}$ & Calasparra (Murcia) & nectarine & MW798307 & MW798348 & MW797022 \\
\hline PR_168 & $\begin{array}{c}\text { Paratylenchus tenuicaudatus } \\
\text { Wu, } 1961\end{array}$ & Sollana (Valencia) & nectarine & MW798308 & MW798349 & MW797023 \\
\hline PR_208 & $\begin{array}{l}\text { Paratylenchus tenuicaudatus } \\
\text { Wu, } 1961\end{array}$ & Sástago (Zaragoza) & apricot & MW798309 & - & - \\
\hline PR_122 & $\begin{array}{c}\text { Paratylenchus veruculatus } \\
\text { Wu, } 1962\end{array}$ & El Moral (Murcia) & almond & MW798310 & - & MW797026 \\
\hline PR_106 & $\begin{array}{c}\text { Paratylenchus veruculatus } \\
\text { Wu, } 1962\end{array}$ & $\begin{array}{c}\text { Santa María de Nieva } \\
\text { (Almería) }\end{array}$ & almond & $\begin{array}{l}\text { MW798311- } \\
\text { MW798312 }\end{array}$ & $\begin{array}{l}\text { MW798350- } \\
\text { MW798351 }\end{array}$ & $\begin{array}{l}\text { MW797027- } \\
\text { MW797029 }\end{array}$ \\
\hline PR_115 & $\begin{array}{l}\text { Paratylenchus veruculatus } \\
\text { Wu, } 1962\end{array}$ & Lúcar (Almería) & almond & MW798313 & MW798352 & MW797024 \\
\hline PR_118 & $\begin{array}{c}\text { Paratylenchus veruculatus } \\
\text { Wu, } 1962\end{array}$ & Serón (Almería) & almond & MW798314 & MW798353 & MW797025 \\
\hline PR_193 & $\begin{array}{l}\text { Paratylenchus veruculatus } \\
\text { Wu, } 1962\end{array}$ & La Almunia (Zaragoza) & cherry & MW798315 & MW798354 & - \\
\hline
\end{tabular}

a Type locality (type specimens).

A total of 231 individuals including 214 females and 17 males were used for morphological and morphometrical analyses. Specimens for study using light microscopy (LM) and morphometrical studies were killed and fixed in an aqueous cold solution of $4 \%$ formaldehyde $+1 \%$ glycerol, dehydrated using alcohol-saturated chamber and processed to pure glycerine using Seinhorst's method [54] as modified by De Grisse [55]. Light micrographs were taken using fresh nematodes and measurements of each nematode population including important diagnostic characteristics (i.e., de Man indices, body length, stylet length, lip region, tail shape) [56] were performed using a Leica DM6 compound microscope with a Leica DFC7000 $\mathrm{T}$ digital camera using fixed and embedded nematodes in glycerin. Nematodes were identified at the species level using an integrative approach combining molecular and morphological techniques to achieve efficient and accurate identification $[21,24,28]$. For each nematode population, key diagnostic characters were determined, including body length, stylet length, a ratio (body length/maximum body width), $\mathrm{b}$ ratio (body length/total pharynx length), $\mathrm{c}$ ratio (body length/tail length), $\mathrm{c}^{\prime}$ ratio (tail length/body width at anus), $\mathrm{V}$ ratio (distance from anterior end to vulva/body length $\times 100$ ), and o ratio (distance from stylet base to dorsal pharyngeal opening/stylet length 100) $[21,24,28]$, and the sequencing of specific DNA fragments (described below) confirmed the identity of the nematode species for each population.

\subsection{Nematode Molecular Characterization}

For molecular analyses, and in order to avoid mistakes in case of mixed populations in the same sample (being common in several soil samples), single specimens from the sample were temporarily mounted in a drop of $1 \mathrm{M} \mathrm{NaCl}$ containing glass beads (to avoid nematode crushing/damaging specimens) to ensure homogenous morphology with specimens conformed with the unidentified population. All necessary morphological and morphometrical data by taking pictures and measurements using the above camera-equipped microscope were recorded. This was followed by DNA extraction from single individuals as described by Palomares-Rius et al. [57], and more importantly, for all the 27 studied isolates, all the three molecular markers of each Paratylenchus isolate belong to the same single extracted individual in each PCR tube without any exception. The D2 and D3 expansion domains of the $28 \mathrm{~S}$ rRNA were amplified using the D2A (5'-ACAAGTACCGTGAGGGAAAGTTG-3 ${ }^{\prime}$ ) and D3B (5'-TCGGAAGGAACCAGCTACTA$\left.3^{\prime}\right)$ primers [58]. The Internal Transcribed Spacer region (ITS) was amplified by using 
forward primer TW81 (5'-GTTTCCGTAGGTGAACCTGC-3') and reverse primer AB28 (5'-ATATGCTTAAGTTCAGCGGGT-3') [59]. The COI gene was amplified using the primers JB3 (5'-TTTTTTGGGCATCCTGAGGTTTAT- $\left.{ }^{\prime}\right)$ and JB5 (5'-AGCACCTAAACTTAAAACAT AATGAAAATG-3') [60]. The PCR cycling conditions for the $28 \mathrm{~S}$ rRNA and ITS regions were as follows: $95^{\circ} \mathrm{C}$ for $15 \mathrm{~min}$, followed by 35 cycles of $94{ }^{\circ} \mathrm{C}$ for $30 \mathrm{~s}$, an annealing temperature of $55^{\circ} \mathrm{C}$ for $45 \mathrm{~s}$, and $72{ }^{\circ} \mathrm{C}$ for $1 \mathrm{~min}$, and 1 final cycle of $72{ }^{\circ} \mathrm{C}$ for $10 \mathrm{~min}$. The PCR cycling for COI primers was as follows: $95{ }^{\circ} \mathrm{C}$ for $15 \mathrm{~min}, 39$ cycles at $94{ }^{\circ} \mathrm{C}$ for $30 \mathrm{~s}, 53{ }^{\circ} \mathrm{C}$ for $30 \mathrm{~s}$, and $68^{\circ} \mathrm{C}$ for $1 \mathrm{~min}$, followed by a final extension at $72{ }^{\circ} \mathrm{C}$ for $7 \mathrm{~min}$. PCR volumes were adapted to $25 \mu \mathrm{L}$ for each reaction, and primer concentrations were as described in De Ley et al. [58], Subbotin et al. [59] and Bowles et al. [60]. We used $5 \times$ HOT FIREpol Blend Master Mix (Solis Biodyne, Tartu, Estonia) in all PCR reactions. The PCR products were purified after amplification using ExoSAP-IT (Affimetrix, USB products, Kandel, Germany) and used for direct sequencing in both directions with the corresponding primers. The resulting products were purified and run in a DNA multicapillary sequencer (Model 3130XL Genetic Analyzer; Applied Biosystems, Foster City, CA, USA), using the BigDye Terminator Sequencing Kit v.3.1 (Applied Bio-systems) at the Stab Vida sequencing facility (Caparica, Portugal). The sequence chromatograms of the 3 markers (ITS, COI and D2-D3 expansion segments of 28S rRNA) were analyzed using DNASTAR LASERGENE SeqMan v. 7.1.0. Basic local alignment search tool (BLAST) at the National Center for Biotechnology Information (NCBI, Bethesda, MD, USA) was used to confirm the species identity of the DNA sequences obtained in this study [61]. The newly obtained sequences were deposited in the GenBank database under accession numbers indicated on the phylogenetic trees and in Table 1.

\subsection{Phylogenetic Analyses}

D2-D3 expansion segments of $28 \mathrm{~S}$ rRNA, ITS rRNA, and COI mtDNA sequences of the 27 Paratylenchus isolates were obtained in this study. These sequences and other sequences from species of Paratylenchus from GenBank were used for phylogenetic analyses. Selection of outgroup taxa for each dataset were based on previously published studies [24,28,29]. Multiple sequence alignments of the different genes were completed using the FFT-NS-2 algorithm of MAFFT V.7.450 [62]. BioEdit program V. 7.2.5 [63] was used for sequence alignments visualization and edited by Gblocks ver. 0.91b [64] in Castresana Laboratory server (http:/ / molevol.cmima.csic.es / castresana/Gblocks_server.html (accessed on 27 March 2021)) using options for a less stringent selection (minimum number of sequences for a conserved or a flanking position: $50 \%$ of the number of sequences +1 ; maximum number of contiguous non-conserved positions: 8 ; minimum length of a block: 5 ; allowed gap positions: with half). Phylogenetic analyses of the sequence datasets were based on Bayesian inference (BI) using MrBayes 3.1.2 [65]. The best-fit model of DNA evolution was achieved using JModelTest V.2.1.7 [66] with the Akaike Information Criterion (AIC). The best-fit model, the base frequency, the proportion of invariable sites, and the gamma distribution shape parameters and substitution rates in the AIC were then used in MrBayes for the phylogenetic analyses. The general time-reversible model with invariable sites and a gamma-shaped distribution (GTR + I + G) for the D2-D3 segments of 28S rRNA and the partial ITS rRNA and the general time-reversible model with a gamma-shaped distribution $(\mathrm{GTR}+\mathrm{G})$ for COI gene, were run with four chains for 4,4 , and $10 \times 10^{6}$ generations, respectively. A combined analysis of the three ribosomal genes was not undertaken due to several sequences not being available for all species. The sampling for Markov chains was carried out at intervals of 100 generations. For each analysis two runs were conducted. After discarding burn-in samples of $30 \%$ and evaluating convergence, the remaining samples were retained for more in-depth analyses. The topologies were used to generate a 50\% majority-rule consensus tree. On each appropriate clade posterior probabilities (PP) were given. FigTree software version v.1.42 [67] was used for visualizing trees from all analyses. 


\section{Results}

Twelve species were identified from 27 isolates of Paratylenchus spp. from 23 soil samples (codified as PR_014, PR_115, PR_118 and PR_193 contain more than one Paratylenchus species) in 18 municipalities in Spain. These populations were morphologically studied in detail and molecular markers for their identification were provided (Table 1). From these, 4 were considered new undescribed species and 8 were already known described species (Table 1). The new species described herein include Paratylenchus caravaquenus sp. nov., Paratylenchus indalus sp. nov., Paratylenchus pedrami sp. nov., and Paratylenchus zurgenerus sp. nov. The already known species included Paratylenchus baldaccii, Paratylenchus enigmaticus Munawar, Yevtushenko, Palomares-Rius \& Castillo, 2021 [21], P. goodeyi, P. hamatus, P. holdemani, P. israelensis, P. tenuicaudatus, and P. veruculatus. Five of latter are considered as first reports for Spain in this work (viz. P. enigmaticus, P. hamatus, P. holdemani, $P$. israelensis and P. veruculatus) and measurements and molecular markers are provided for their unequivocal identification.

\subsection{Systematics}

3.1.1. Description of Paratylenchus caravaquenus sp. nov.

(Figures 1 and 2, Table 2). http: / / zoobank.org/NomenclaturalActs/50830BAE-BCB4 -4465-98D2-27598AB19E61 (accessed on 27 March 2021).

Female: Body slender, ventrally arcuate to form an open, C-shaped body habitus when heat relaxed; cuticle finely annulated; lateral field equidistant with four distinct lines; lip region conoid rounded, with anterior end flattened, continuous with the rest of the body, small submedian lobes or almost indistinguishable in some specimens. Labial framework sclerotization weak; pharyngeal region typical paratylenchoid type. Stylet rigid, straight; stylet knobs rounded; dorsal pharyngeal gland opening 5.5-8.0 $\mu \mathrm{m}$ behind stylet knobs. Median pharyngeal bulb slender elongate, bearing distinct large valves; isthmus short slender, surrounded by nerve ring; basal bulb pyriform, pharyngeal-intestinal valve rounded; excretory pore situated at the level or anterior to pharyngeal basal bulb. Hemizonid 1-2 annuli long, situated immediately anterior to excretory pore. Body slightly narrower posterior to vulva; ovary outstretched, well developed; spermatheca and crustaformeria well developed; spermatheca rounded; vulva a transverse slit occupying half of the corresponding body width. Vulval lips prominent, the anterior lip is protruding further than the posterior lip; advulval flaps present, but not prominent in fresh specimens. Anus difficult to distinguish in some specimens; tail slender, conoid, finely annulated, and gradually tapers to form a rounded or subacute terminus in some individuals.

Male: Body slender than female, tapering towards both ends, posterior region ventrally arcuate when heat relaxed. Cuticle apparently smooth with fine annulations; labial region similar to that of female but narrower and slightly truncated, continuous with body, sclerotization in labial region weak; stylet lacking. Pharynx rudimentary and non-functional, procorpus, metacorpus, and basal bulb inconspicuous; excretory pore located $73.0 \mu \mathrm{m}$ away from anterior end. Testis outstretched, with small spermatozoa; spicule slender, slightly curved towards end; gubernaculum curved; bursa absent. Tail elongate-conoid, tapering gradually to a finely pointed tip.

Juveniles: It was the most abundant developmental stage at the end of summer in the type locality, most probably J4. They were similar in morphology to the adult females. However, they are characterized by the presence of weak stylet; underdeveloped pharynx components; underdeveloped genital primordium; indistinct anus; and posterior body with a rounded terminus.

\section{Diagnosis and Relationships}

The new species can be characterized by the presence of 4 lateral lines in lateral field, advulval flaps present, and a moderate female stylet length of 29.8 (26.5-32.0) $\mu \mathrm{m}$. Lip region conoid-rounded, with the anterior end flattened, continuous with the rest of the body. Excretory pore situated at the level or anterior to the pharyngeal basal bulb. Spermatheca 
rounded. Tail elongate-conoid gradually tapering to form a rounded terminus. According to species grouping by Ghaderi et al. [36] belongs to group 3 characterized by stylet length less than $40 \mu \mathrm{m}$, four lateral lines and advulval flaps present.
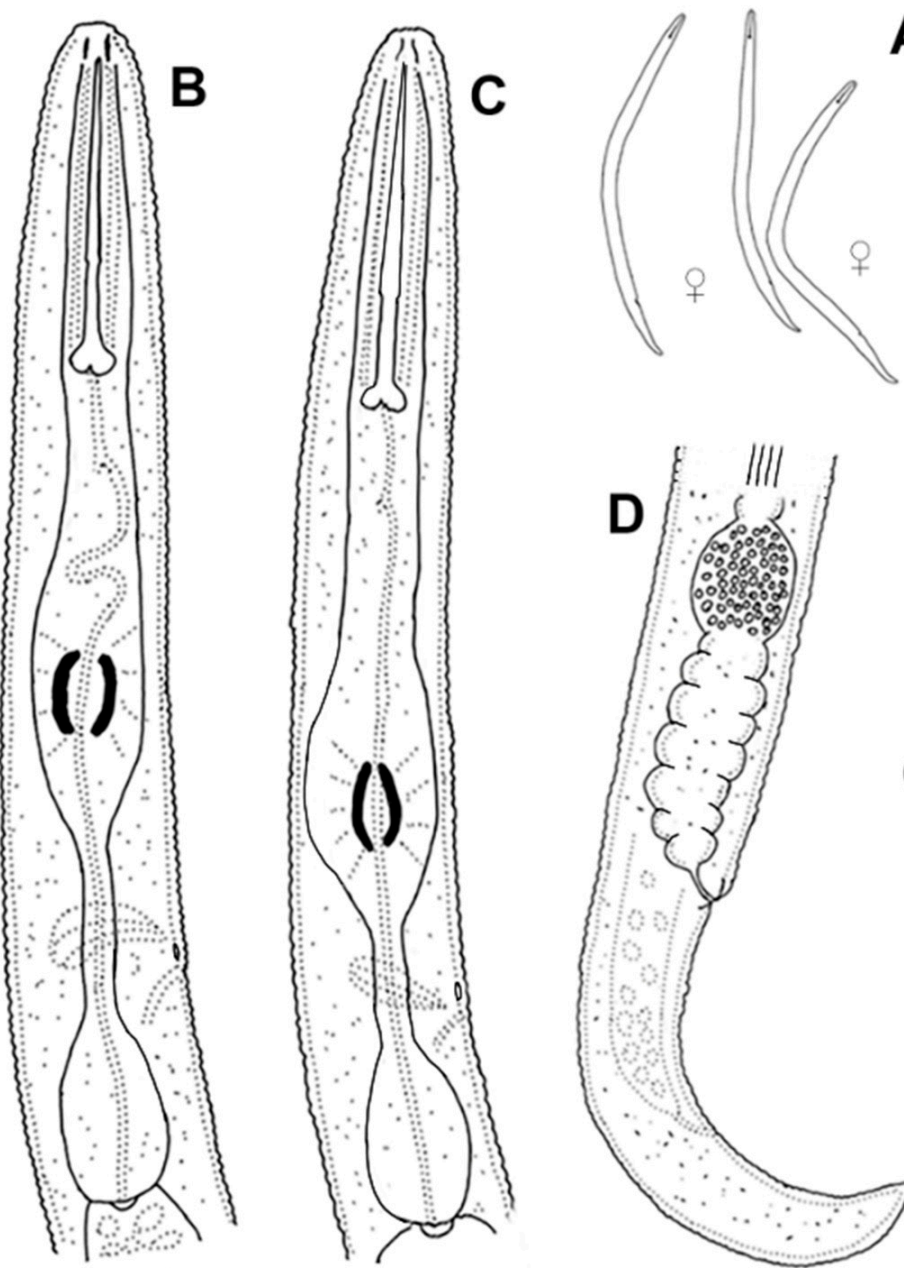

A
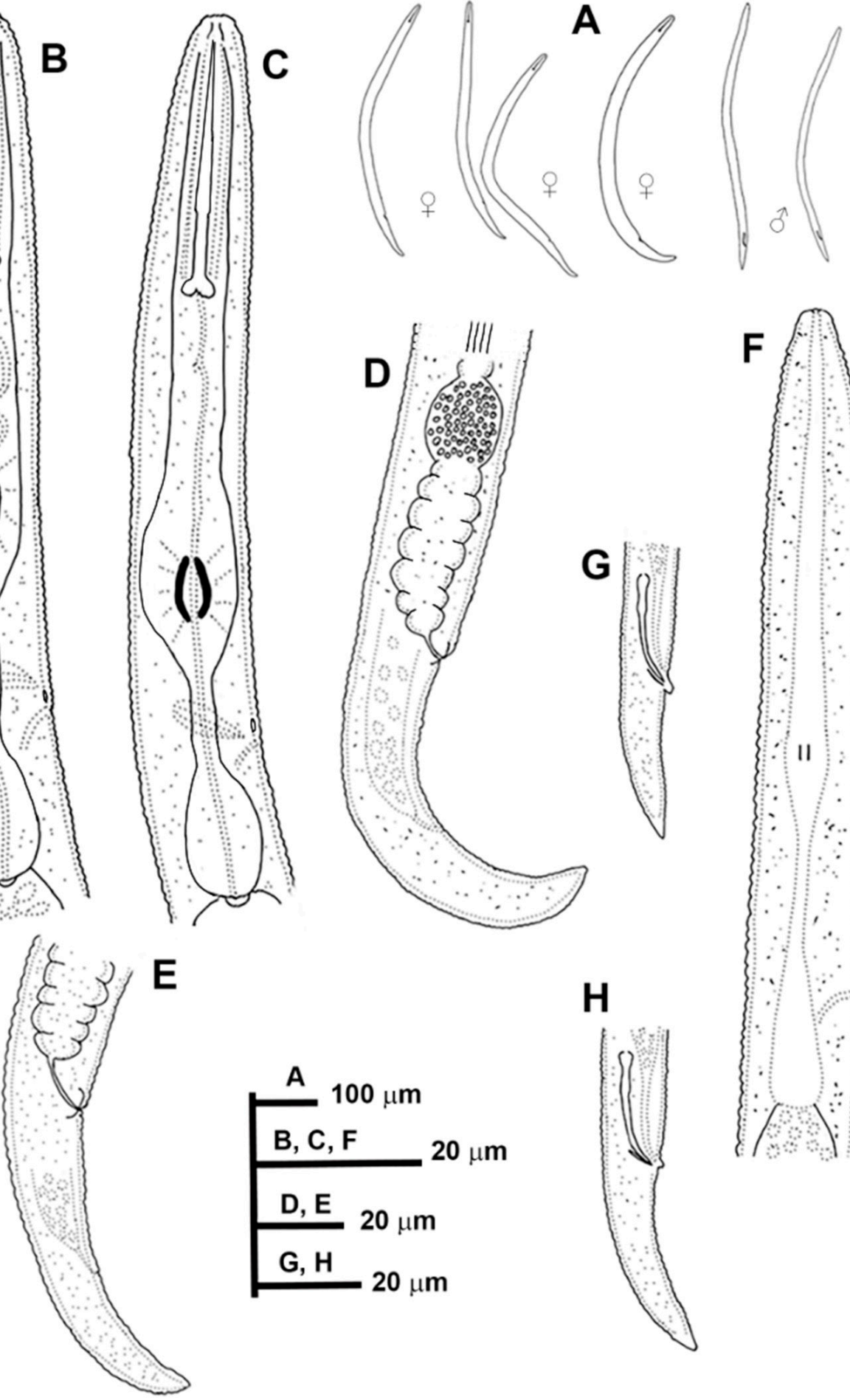

H
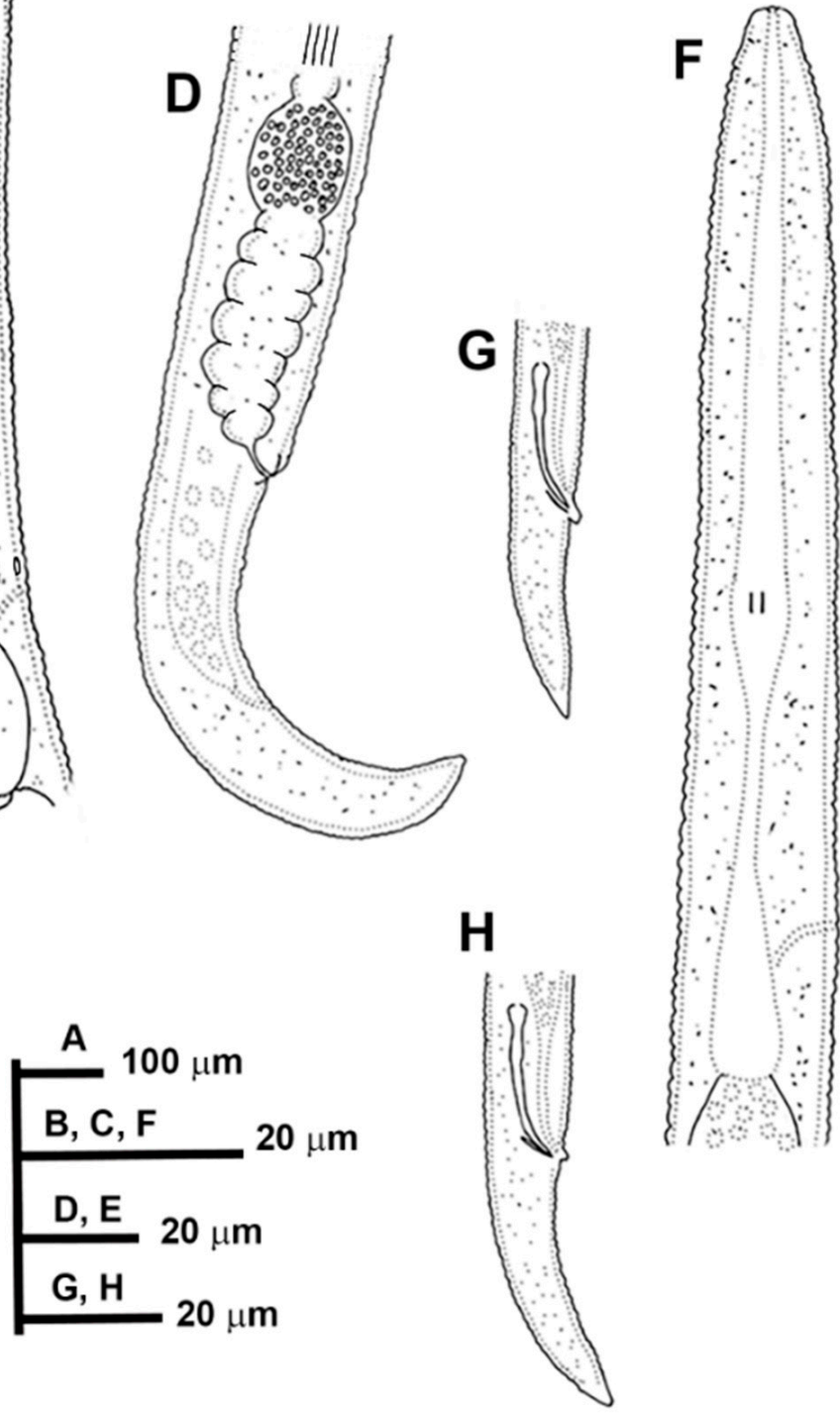

Figure 1. Line drawings of Paratylenchus caravaquenus sp. nov. (A): Entire females and males; (B,C): Female pharyngeal region; (D,E): Female posterior region; (F): Male pharyngeal region showing absence of stylet; $(\mathbf{G}, \mathbf{H})$ : Male posterior region. 

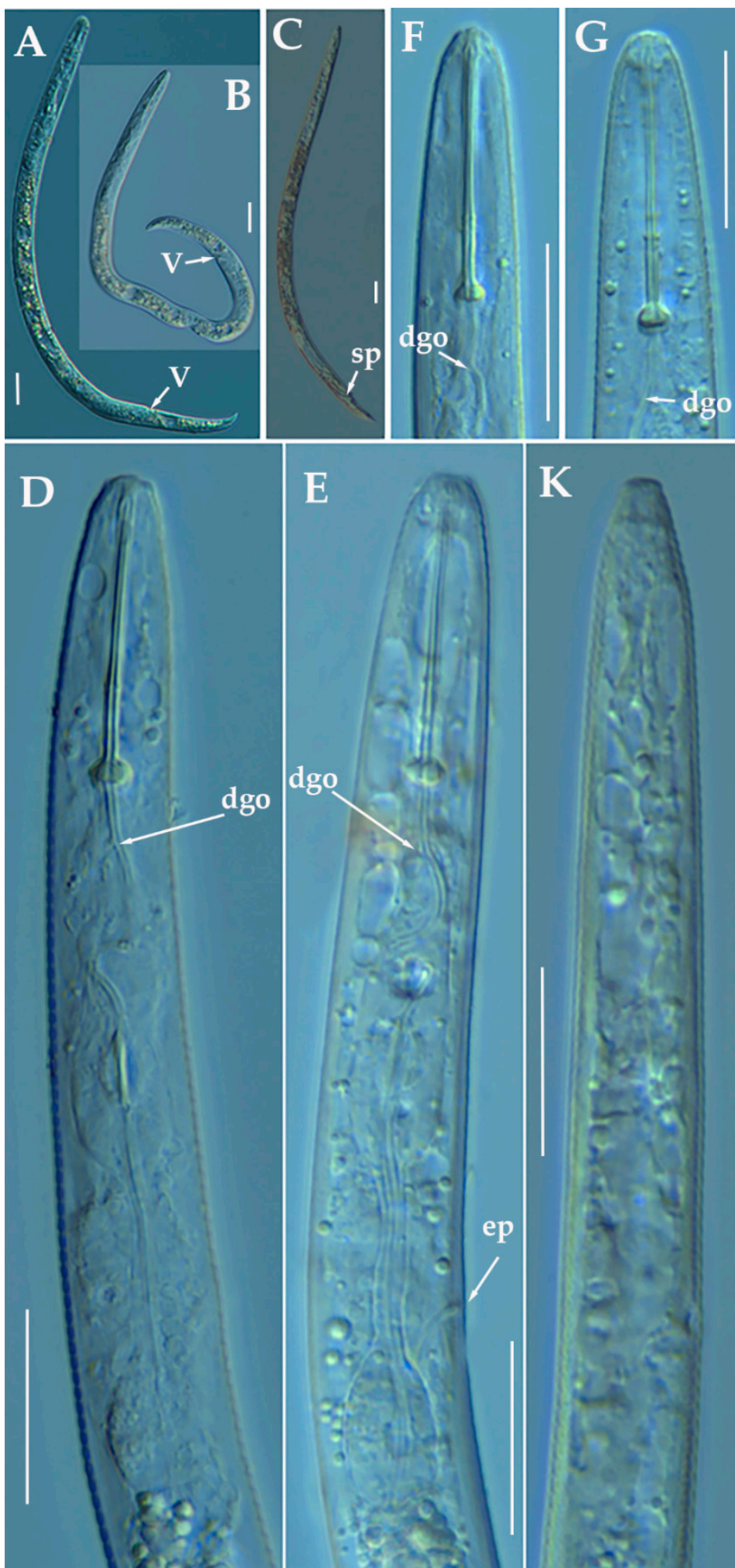
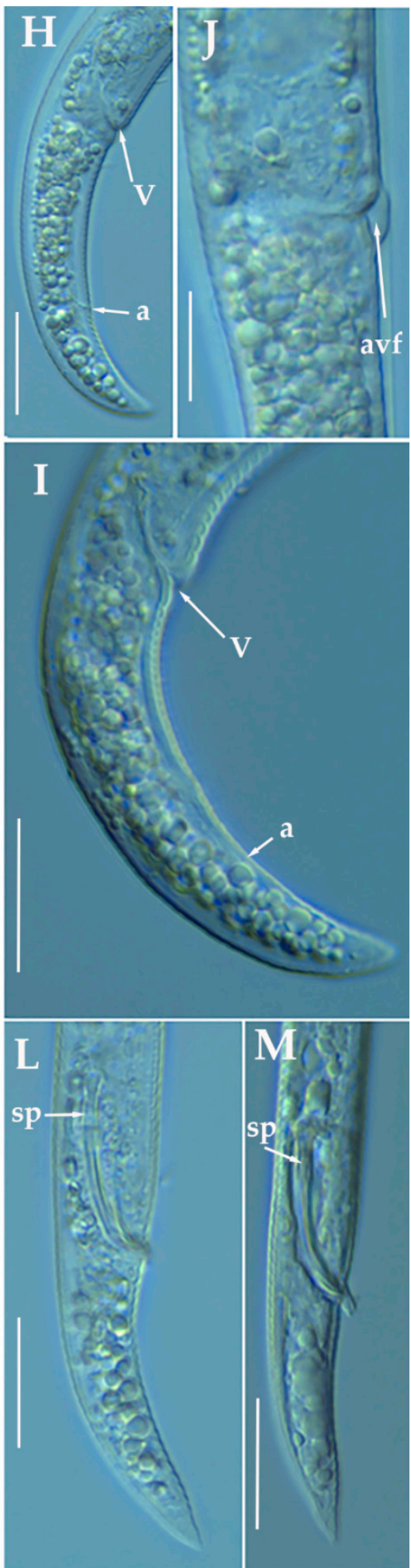

Figure 2. Light photomicrographs of Paratylenchus caravaquenus sp. nov. (A,B): Entire female with vulva arrowed; (C): Entire male with spicules arrowed; (D,E): Female pharyngeal region; $(\mathbf{F}, \mathbf{G})$ : Female lip region; $(\mathbf{H}, \mathbf{I})$ : Female posterior region with vulva and anus (arrowed); (J): Detail of vulva showing advulval flap (arrowed); (K): Male pharyngeal region showing absence of stylet; (L,M): Male posterior region showing spicules (arrowed). Scale bars $(\mathbf{A}-\mathbf{I}, \mathbf{K}-\mathbf{M}=20 \mu \mathrm{m} ; \mathbf{J}=10 \mu \mathrm{m})$. (Abbreviations: $\mathrm{a}=$ anus; $\mathrm{avf}=$ advulval flap; $\mathrm{dgo}=$ pharyngeal dorsal gland orifice; $\mathrm{ep}=$ excretory pore; $\mathrm{sp}=\mathrm{spicules;}$ $\mathrm{V}=$ vulva). 
Table 2. Morphometrics of Paratylenchus caravaquenus sp. nov. paratype females and males. All measurements are in $\mu \mathrm{m}$ and in the form: mean \pm s.d. (range).

\begin{tabular}{|c|c|c|c|}
\hline $\begin{array}{c}\text { Measurements } \\
\text { and Ratios }\end{array}$ & $\begin{array}{l}\text { Holotype } \\
\text { Female }\end{array}$ & $\begin{array}{l}\text { Paratype } \\
\text { Females }\end{array}$ & Paratype Males \\
\hline Sample code & PI_ARC & PI_ARC & PI_ARC \\
\hline $\begin{array}{l}\text { Locality } \\
\mathrm{n}\end{array}$ & $\begin{array}{c}\text { Caravaca, Murcia } \\
1\end{array}$ & $\begin{array}{c}\text { Caravaca, Murcia } \\
19\end{array}$ & $\begin{array}{c}\text { Caravaca, Murcia } \\
6\end{array}$ \\
\hline $\mathrm{L}$ & 384 & $384.8 \pm 24.4(344-443)$ & $\begin{array}{c}419 \pm 26.0 \\
(372.5-451.5)\end{array}$ \\
\hline$a^{*}$ & 24.8 & $22.6 \pm 2.3(18.4-27.0)$ & $29.2 \pm 2.7(26.6-33.4)$ \\
\hline $\mathrm{b}$ & 3.5 & $3.7 \pm 0.4(3.2-4.9)$ & $4.4 \pm 0.7(3.7-5.1)$ \\
\hline c & 15.7 & $13.9 \pm 1.0(11.2-16.2)$ & $10.96 \pm 0.5(10.5-11.7)$ \\
\hline$c^{\prime}$ & 2.7 & $2.8 \pm 0.2(2.5-3.2)$ & $3.6 \pm 0.2(3.3-3.9)$ \\
\hline $\mathrm{V}$ or $\mathrm{T}$ & 83.6 & $83.4 \pm 0.7(81.8-84.5)$ & 49.5 \\
\hline G1 & 33.6 & $36.0 \pm 4.5(29.7-49.0)$ & - \\
\hline Stylet length & 28.0 & $29.8 \pm 1.5(26.5-32)$ & - \\
\hline Conus length & 18.0 & $19.5 \pm 1.5(16.5-21)$ & - \\
\hline $\mathrm{m}$ & 64.3 & $65.3 \pm 2.6(60.0-70.0)$ & - \\
\hline DGO & 7.5 & $6.8 \pm 0.8(5.5-8)$ & - \\
\hline $\mathrm{O}$ & 26.8 & $22.9 \pm 2.9(17.2-30.2)$ & - \\
\hline Lip width & 6.0 & $6.1 \pm 0.5(5.5-7.0)$ & $4.7 \pm 0.3(4.5-5.0)$ \\
\hline Median bulb length & 23.0 & $22.1 \pm 3.5(18.0-29.0)$ & - \\
\hline Median bulb width & 9.0 & $10.1 \pm 1.1(9.0-13.5)$ & - \\
\hline $\begin{array}{l}\text { Anterior end to center } \\
\text { median bulb }\end{array}$ & 64.5 & $60.5 \pm 5.5(47.0-72.0)$ & 50.5 \\
\hline $\mathrm{MB}$ & 58.6 & $57.9 \pm 2.9(52.2-64.4)$ & - \\
\hline $\begin{array}{l}\text { Nerve ring to } \\
\text { anterior end }\end{array}$ & 84.0 & $80.9 \pm 7.8(65.0-96.0)$ & - \\
\hline $\begin{array}{l}\text { Excretory pore to } \\
\text { anterior end }\end{array}$ & 90.0 & $86.9 \pm 4.8(77.0-98.0)$ & 73.0 \\
\hline Pharynx length & 110.0 & $\begin{array}{c}104.7 \pm 8.6 \\
(89.0-124.0)\end{array}$ & $94.8 \pm 5.8(88.8-100)$ \\
\hline Maximum body diam. & 15.5 & $17.2 \pm 1.9(14.5-21.0)$ & $14.5 \pm 1.2(13.5-16.0)$ \\
\hline Tail length & 24.5 & $27.9 \pm 2.3(25.0-32.0)$ & $38.3 \pm 1.9(35.5-40.5)$ \\
\hline Anal body diam. & 9.0 & $10.1 \pm 0.6(9.0-11.0)$ & $10.7 \pm 0.8(9.5-11.5)$ \\
\hline Spicules & - & - & $23.3 \pm 0.5(22.5-24.0)$ \\
\hline Gubernaculum & - & - & $5.3 \pm 0.4(5.0-6.0)$ \\
\hline
\end{tabular}

* Abbreviations: $\mathrm{a}$ = body length/greatest body diameter; $\mathrm{b}=$ body length/distance from anterior end to pharyngo-intestinal junction; DGO = distance between stylet base and orifice of dorsal pharyngeal gland; $c=$ body length/tail length; $\mathrm{c}^{\prime}=$ tail length/tail diameter at anus or cloaca; $\mathrm{G} 1=$ anterior genital branch length expressed as percentage $(\%)$ of the body length; $\mathrm{L}=$ overall body length; $\mathrm{m}=$ length of conus as percentage of total stylet length; $\mathrm{MB}=$ distance between anterior end of body and center of median pharyngeal bulb expressed as percentage $(\%)$ of the pharynx length; $\mathrm{n}=$ number of specimens on which measurements are based; $\mathrm{O}=\mathrm{DGO}$ as percentage of stylet length; $\mathrm{T}=$ distance from cloacal aperture to anterior end of testis expressed as percentage (\%) of the body length; $\mathrm{V}=$ distance from body anterior end to vulva expressed as percentage $(\%)$ of the body length.

Morphologically and morphometrically, the new species is close to P. baldaccii, $P$. salubris Raski, 1975, P. coronatus Colbran, 1975 and P. mimulus Raski, 1975. Paratylenchus caravaquenus sp. nov. differs from $P$. baldaccii in having males without stylet vs. males with stylet, other morphometrical characters are in the same range. From a molecular point of view, this species differs from other populations identified as $P$. baldaccii in all the molecular markers studied (D2-D3, ITS and COI). Paratylenchus caravaquenus sp. nov. differs from $P$. salubris in tail shape acute to finely rounded vs. usually bluntly rounded, longer female adults (344-443 vs. 200-250 $\mu \mathrm{m}$ ), and posterior position of the vulva (V) (81.8-84.5\% vs. $78-82 \%$ ). Paratylenchus caravaquenus sp. nov. differs from P. coronatus by longer body in females ( $344-443$ vs. $270-300 \mu \mathrm{m})$, shorter stylet (26.5-32.0 vs. $31-39 \mu \mathrm{m}$ ) and posterior vulva position ( $82-84 \%$ vs. $78-82 \%$ ). Paratylenchus caravaquenus sp. nov. differs from P. mimulus in longer body in females (344-443 $\mu \mathrm{m}$ vs. $180-260 \mu \mathrm{m})$ and posterior vulva position $(82-84 \%$ vs. $78-82 \%)$. Other species related phylogenetically 
with morphological and morphometrical data as P. holdemani differs by a shorter body (344-443 $\mu \mathrm{m}$ vs. $290-350 \mu \mathrm{m})$, shorter stylet $(26.5-32.0 \mu \mathrm{m}$ vs. $21-23 \mu \mathrm{m})$, posterior vulva $(81.4-84.5 \%$ vs. $84-86 \%)$ and males without stylet vs. males with stylet.

\section{Molecular Characterization}

Three D2-D3 of 28S (MW798270-MW798272), three ITS (MW798316-MW798318), and two COI gene sequences (MW797003-MW797004) were generated for this new species without intraspecific sequence variations. Paratylenchus caravaquenus sp. nov. is $97 \%$ similar for the D2-D3 region (22 nucleotides and no indels) to P. nawadus (MN088373), however, it can be readily morphologically distinguished by longer stylet $(26.5-32.0 \mu \mathrm{m}$ vs. 18.7-22 $\mu \mathrm{m}$ ), and the presence of a truncated lip region with well-developed submedian lobes in P. nawadus [27]. Unfortunately, no data for ITS or COI from P. nawadus are available in the GenBank. From our P. baldaccii population (PR_152), P. caravaquenus sp. nov. is $79 \%, 79 \%$, and $84 \%$ similar in D2-D3 region, ITS and COI sequences, respectively, clearly separating both species.

\section{Remarks}

This species has been found in only one forest close to an almond field. The population presented moderate numbers of individuals in soil (224 individuals $/ 500 \mathrm{~cm}^{3}$ of soil), but the majority of them were J4 individuals.

Type Habitat and Locality

Paratylenchus caravaquenus sp. nov. was found in the rhizosphere of a Pinus halepensis Mill., 1768 forest (coordinates $38^{\circ} 04^{\prime} 52.6^{\prime \prime} \mathrm{N} ; 2^{\circ} 02^{\prime} 17.0^{\prime \prime} \mathrm{W}$ ); the municipal district of Caravaca, Murcia, Spain.

\section{Etymology}

The species epithet, caravaquenus, refers to the name of the type locality (Caravaca).

Type Material

Holotype female, 17 paratypes females and 6 male paratypes (slide numbers PI_AR-01 to PI_AR-11) were deposited in the Nematode Collection of the Institute for Sustainable Agriculture, CSIC, Córdoba, Spain, and two females deposited at the USDA Nematode Collection (slide T-7479p).

\subsubsection{Description of Paratylenchus indalus sp. nov.}

(Figures 3-5; Table 3). http:/ / zoobank.org/NomenclaturalActs/AD30DC56--76E0-4 741-A38A-52734155F641 (accessed on 27 March 2021).

Female: Body slender, ventrally arcuate to form an open C-shaped when heat relaxed. Cuticle finely annulated; lateral field equidistant with four distinct lines. Lip region rounded, with anterior end flattened, continuous with the rest of the body, presence of small submedian lobes; labial framework sclerotization weak. Pharyngeal region typical paratylenchoid type; stylet rigid, straight; stylet knobs rounded; dorsal pharyngeal gland opening 5.5-6.5 $\mu \mathrm{m}$ behind stylet knobs. Median pharyngeal bulb slender elongate, bearing distinct large valves; isthmus short slender, surrounded by nerve ring; basal bulb pyriform, pharyngeal-intestinal valve rounded; excretory pore situated anterior to pharyngeal basal bulb. Hemizonid 1-2 annuli long situated immediately anterior to excretory pore; body slightly narrower posterior to vulva; ovary outstretched, well developed. Spermatheca and crustaformeria well developed; spermatheca rounded; vulva a transverse slit occupying half of the corresponding body width; vulval lips prominent, the anterior lip is protruding further than the posterior lip; advulval flaps present, but not prominent in fresh specimens. Anus difficult to distinguish. Tail slender, conoid, finely annulated, and gradually tapers to form a rounded terminus or pointed in some individuals (Figure 5). 

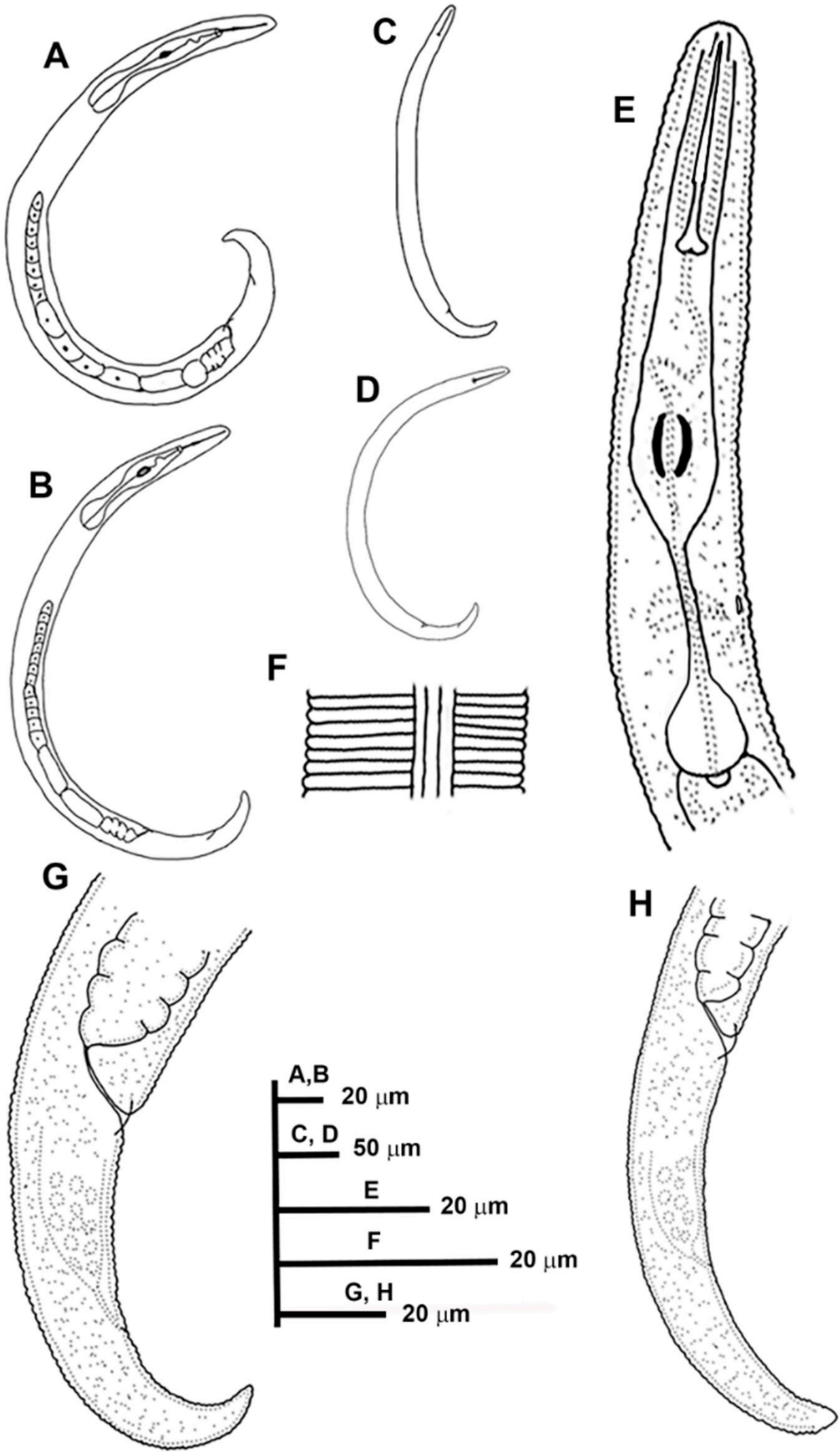

Figure 3. Line drawings of Paratylenchus indalus sp. nov. (A-D): Entire females; (E): Female pharyngeal region; $(\mathbf{F})$ : Lateral field at mid-body; $(\mathbf{G}, \mathbf{H})$ : Female posterior region. 

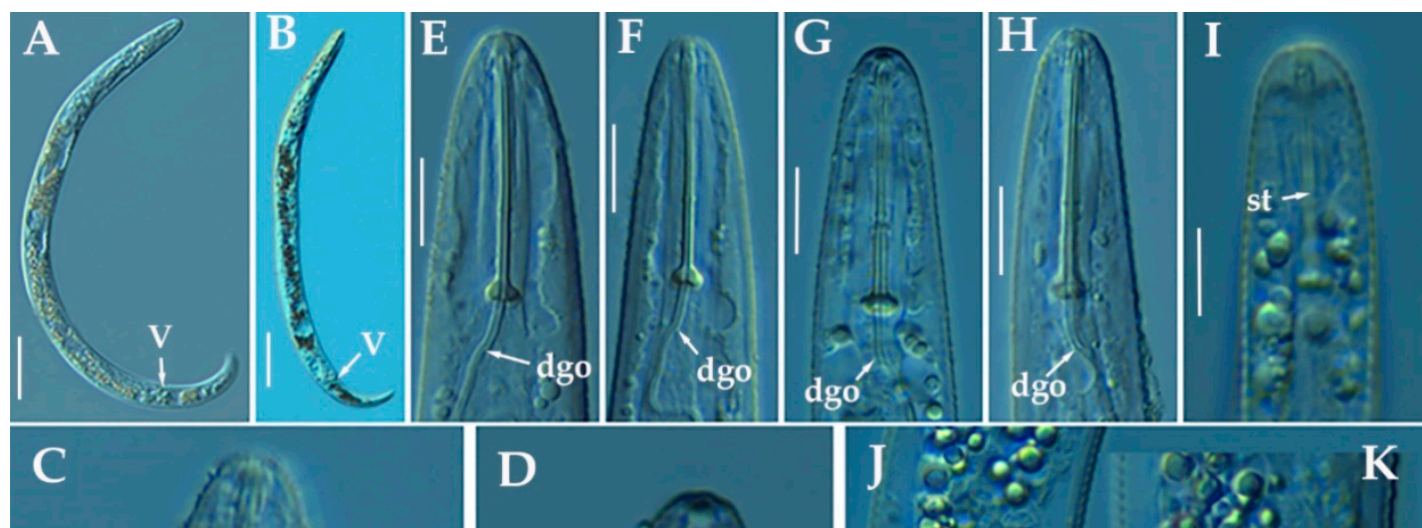

D
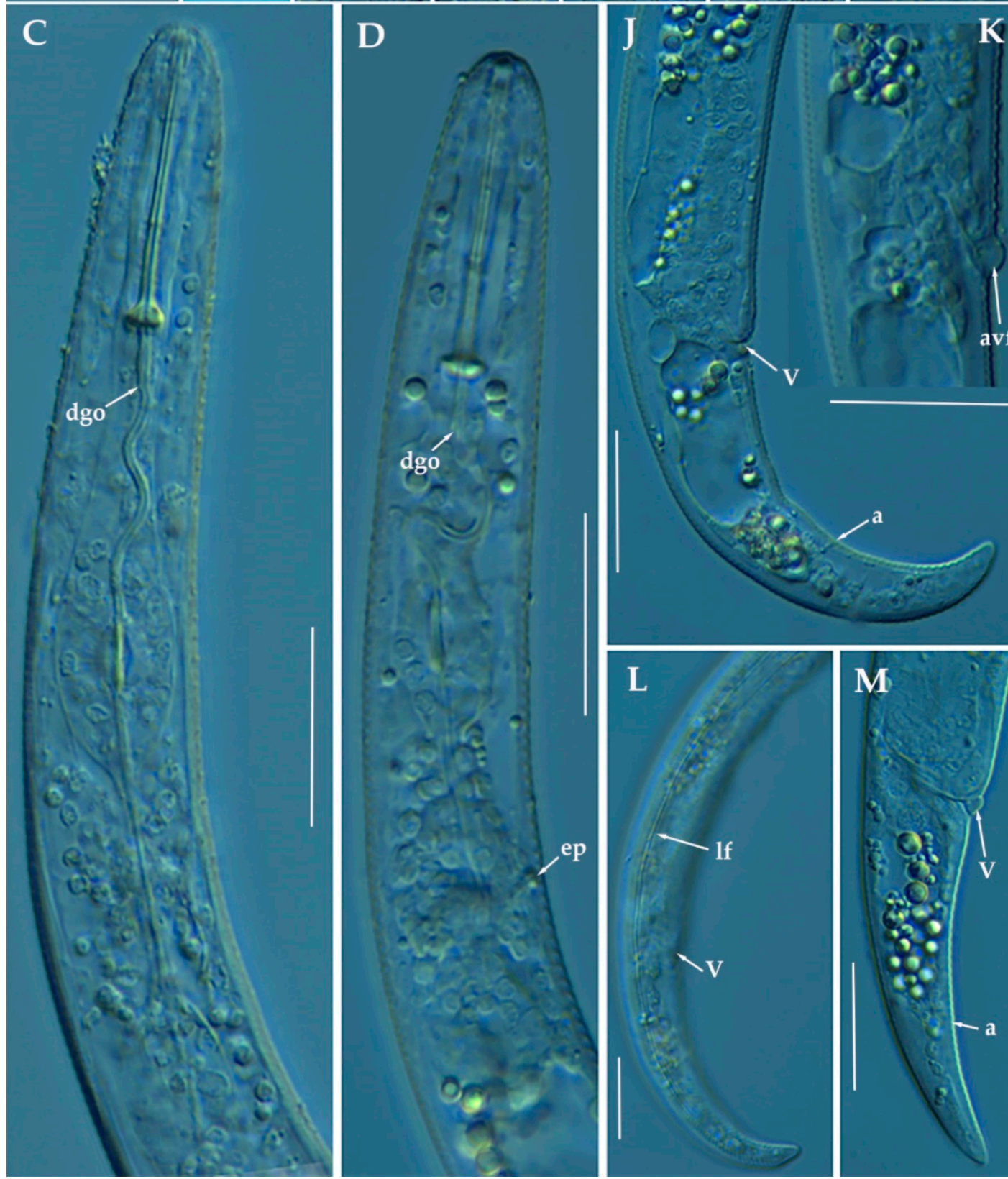

$\mathbf{K}$

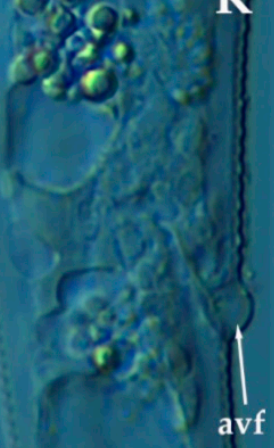

avf

Figure 4. Light photomicrographs of female of Paratylenchus indalus sp. nov. (A,B): Entire female with vulva arrowed; (C,D): Female pharyngeal region; (E-H): Female lip region; (I): Fourth-stage juvenile showing stylet (arrowed); (J): Female posterior region with vulva and anus (arrowed); (K): Detail of vulva showing advulval flap (arrowed); (L,M): Female posterior region with lateral field, vulva and anus (arrowed). Scale bars (A-D, J-M $=20 \mu \mathrm{m} ; \mathbf{E}-\mathbf{I}=10 \mu \mathrm{m})$. (Abbreviations: $\mathrm{a}=$ anus; $\mathrm{avf}=\mathrm{advulval}$ flap; dgo = pharyngeal dorsal gland orifice; ep = excretory pore; lf = lateral field; $\mathrm{V}=\mathrm{vulva}$ ). 
Table 3. Morphometrics of Paratylenchus indalus sp. nov. paratype females and other populations from Spain. All measurements are in $\mu \mathrm{m}$ and in the form: mean \pm s.d. (range).

\begin{tabular}{|c|c|c|c|c|c|}
\hline Measurements and Ratios & Holotype Female & $\begin{array}{l}\text { Paratype } \\
\text { Females }\end{array}$ & Females & Females & Females \\
\hline Sample code & PR_104 & PR_104 & PR_114 & PR_118 & PR_119 \\
\hline Locality & $\begin{array}{l}\text { Sta. } M^{\text {a }} \text { Nieva, } \\
\text { Almería }\end{array}$ & $\begin{array}{l}\text { Sta. } M^{a} \text { Nieva, } \\
\text { Almería }\end{array}$ & $\begin{array}{l}\text { Urracal, } \\
\text { Almería }\end{array}$ & Serón, Almería & El Hijate, Almería \\
\hline $\mathrm{n}$ & 1 & 13 & 4 & 2 & 3 \\
\hline $\mathrm{L}$ & 377 & $\begin{array}{c}377.8 \pm 36.3 \\
(317-434)\end{array}$ & $\begin{array}{c}450.5 \pm 47.9 \\
(382-484)\end{array}$ & 370,399 & $\begin{array}{l}385 \pm 26.2 \\
(357-409)\end{array}$ \\
\hline$a^{*}$ & 22.2 & $\begin{array}{c}19.4 \pm 2.3 \\
(16.0-23.7)\end{array}$ & $\begin{array}{l}24.0 \pm 2.3 \\
(21.2-26.8)\end{array}$ & $19.5,20.6$ & $\begin{array}{l}20.7 \pm 1.9 \\
(19.5-22.9)\end{array}$ \\
\hline $\mathrm{b}$ & 3.9 & $\begin{array}{l}3.7 \pm 0.3 \\
(3.3-4.2)\end{array}$ & $\begin{array}{l}4.5 \pm 0.3 \\
(4.1-4.7)\end{array}$ & $3.6,3.8$ & $\begin{array}{l}3.7 \pm 0.2 \\
(3.4-3.9)\end{array}$ \\
\hline c & 12.6 & $\begin{array}{l}12.0 \pm 1.6 \\
(9.0-14.8)\end{array}$ & $\begin{array}{l}16.0 \pm 1.9 \\
(13.2-17.3)\end{array}$ & $13.0,13.8$ & $\begin{array}{c}12.5 \pm 1.2 \\
(11.2-13.4)\end{array}$ \\
\hline$c^{\prime}$ & 3.0 & $\begin{array}{l}3.0 \pm 0.1 \\
(2.7-3.1)\end{array}$ & $\begin{array}{l}3.0 \pm 0.2 \\
(2.8-3.2)\end{array}$ & $2.8,2.9$ & $\begin{array}{l}3.0 \pm 0.2 \\
(2.9-3.2)\end{array}$ \\
\hline $\mathrm{V}$ & 83.0 & $\begin{array}{l}82.9 \pm 1.4 \\
(80.4-84.5)\end{array}$ & $\begin{array}{l}81.6 \pm 1.1 \\
(80.6-83.0)\end{array}$ & $82.4,82.7$ & $\begin{array}{l}83.4 \pm 0.6 \\
(83.0-84.1)\end{array}$ \\
\hline G1 & 20.4 & $\begin{array}{l}25.0 \pm 4.9 \\
(20.2-38.8)\end{array}$ & $\begin{array}{l}33.5 \pm 7.6 \\
(28.3-44.5)\end{array}$ & $37.0,39.8$ & $\begin{array}{l}29.2 \pm 7.7 \\
(24.6-38.0)\end{array}$ \\
\hline Stylet length & 30.0 & $\begin{array}{l}28.3 \pm 0.9 \\
(26.0-29.5)\end{array}$ & $\begin{array}{l}31.0 \pm 1.4 \\
(29.0-32.0)\end{array}$ & $28.5,29.0$ & $\begin{array}{l}28.8 \pm 1.0 \\
(28.0-30.0)\end{array}$ \\
\hline Conus length & 19.0 & $\begin{array}{l}18.3 \pm 0.6 \\
(17.0-19.0)\end{array}$ & $\begin{array}{l}18.1 \pm 0.6 \\
(17.5-19.0)\end{array}$ & $17.5,19.0$ & $\begin{array}{l}18.7 \pm 1.2 \\
(18.0-20.0)\end{array}$ \\
\hline $\mathrm{m}$ & 63.3 & $\begin{array}{l}64.5 \pm 1.6 \\
(62.1-66.7)\end{array}$ & $\begin{array}{l}58.6 \pm 4.8 \\
(54.7-65.5)\end{array}$ & $61.4,65.5$ & $\begin{array}{c}64.7 \pm 1.8 \\
(63.2-66.7)\end{array}$ \\
\hline DGO & 6.5 & $\begin{array}{l}6.0 \pm 0.4 \\
(5.5-6.5)\end{array}$ & $\begin{array}{l}6.3 \pm 0.3 \\
(6.0-6.5)\end{array}$ & $6.5,6.5$ & $\begin{array}{l}5.5 \pm 0.0 \\
(5.5-5.5)\end{array}$ \\
\hline $\mathrm{O}$ & 21.7 & $\begin{array}{l}21.1 \pm 1.7 \\
(18.6-23.2)\end{array}$ & $\begin{array}{l}20.2 \pm 1.6 \\
(18.8-22.4)\end{array}$ & $22.4,22.8$ & $\begin{array}{l}19.5 \pm 0.2 \\
(19.3-19.6)\end{array}$ \\
\hline Lip width & 6.0 & $\begin{array}{l}6.2 \pm 0.3 \\
(5.5-6.5)\end{array}$ & $\begin{array}{l}6.5 \pm 0.4 \\
(6.0-7.0)\end{array}$ & $6.5,7.0$ & $\begin{array}{l}6.5 \pm 0.5 \\
(6.0-7.0)\end{array}$ \\
\hline Median bulb length & 21.0 & $\begin{array}{l}20.7 \pm 2.0 \\
(18.0-24.0)\end{array}$ & - & 28.0 & - \\
\hline Median bulb width & 11.0 & $\begin{array}{l}10.0 \pm 1.0 \\
(9.0-12.0)\end{array}$ & - & 12.0 & - \\
\hline $\begin{array}{l}\text { Anterior end to center } \\
\text { median bulb }\end{array}$ & 60.0 & $\begin{array}{l}56.4 \pm 3.5 \\
\quad(51-65)\end{array}$ & $\begin{array}{l}55.0 \pm 0.8 \\
(54.0-56.0)\end{array}$ & $55.0,56.0$ & $\begin{array}{l}58.3 \pm 1.5 \\
(57.0-60.0)\end{array}$ \\
\hline $\mathrm{MB}$ & 62.5 & $\begin{array}{l}55.2 \pm 2.3 \\
(51.2-59.0)\end{array}$ & $\begin{array}{l}54.7 \pm 2.6 \\
(53.3-58.5)\end{array}$ & $53.3,53.4$ & $\begin{array}{l}55.4 \pm 1.7 \\
(54.3-57.4)\end{array}$ \\
\hline Nerve ring to anterior end & 72.0 & $\begin{array}{l}76.7 \pm 6.4 \\
(68.0-92.0)\end{array}$ & $\begin{array}{l}70.5 \pm 1.3 \\
(69.0-72.0)\end{array}$ & $71.0,72.0$ & $\begin{array}{l}76.0 \pm 7.0 \\
(71.0-84.0)\end{array}$ \\
\hline $\begin{array}{l}\text { Excretory pore to } \\
\text { anterior end }\end{array}$ & 86.0 & $\begin{array}{c}91.1 \pm 11.0 \\
(79.0-118.0)\end{array}$ & $\begin{array}{c}87.0 \pm 2.6 \\
(84.0-90.0)\end{array}$ & $86.0,90.0$ & $\begin{array}{c}91.3 \pm 8.4 \\
(86.0-101.0)\end{array}$ \\
\hline Pharynx length & 96.0 & $\begin{array}{l}101.5 \pm 9.8 \\
(89.0-127.0)\end{array}$ & $\begin{array}{l}100.8 \pm 4.8 \\
(94.0-105.0)\end{array}$ & $103.0,105.0$ & $\begin{array}{c}105.3 \pm 4.5 \\
(101.1-110.0)\end{array}$ \\
\hline Maximum body diam. & 17.0 & $\begin{array}{c}19.7 \pm 3.0 \\
(14.0-24.0)\end{array}$ & $\begin{array}{c}18.8 \pm 1.0 \\
(18.0-20.0)\end{array}$ & $18.0,20.5$ & $\begin{array}{c}18.7 \pm 2.1 \\
(17.0-21.0)\end{array}$ \\
\hline Tail length & 30.0 & $\begin{array}{l}32.0 \pm 5.3 \\
(27.0-47.0)\end{array}$ & $\begin{array}{l}28.3 \pm 1.0 \\
(27.0-29.0)\end{array}$ & $28.5,29.0$ & $\begin{array}{l}31.0 \pm 1.7 \\
(29.0-32.0)\end{array}$ \\
\hline Anal body diam. & 10.0 & $\begin{array}{l}10.8 \pm 1.5 \\
(9.5-15.0)\end{array}$ & $\begin{array}{c}9.5 \pm 0.7 \\
(9.0-10.5)\end{array}$ & $10.0,10.5$ & $\begin{array}{c}10.3 \pm 0.6 \\
(10.0-11.0)\end{array}$ \\
\hline
\end{tabular}

* Abbreviations: $\mathrm{a}=$ body length/greatest body diameter; $\mathrm{b}=$ body length/distance from anterior end to pharyngo-intestinal junction; $\mathrm{DGO}=$ distance between stylet base and orifice of dorsal pharyngeal gland; $\mathrm{c}=$ body length/tail length; $\mathrm{c}^{\prime}=$ tail length/tail diameter at anus or cloaca; G1 = anterior genital branch length expressed as percentage (\%) of the body length; $\mathrm{L}=$ overall body length; $\mathrm{m}=$ length of conus as percentage of total stylet length; $\mathrm{MB}=$ distance between anterior end of body and center of median pharyngeal bulb expressed as percentage $(\%)$ of the pharynx length; $\mathrm{n}=$ number of specimens on which measurements are based; $\mathrm{O}=\mathrm{DGO}$ as percentage of stylet length; $\mathrm{V}=$ distance from body anterior end to vulva expressed as percentage (\%) of the body length. 

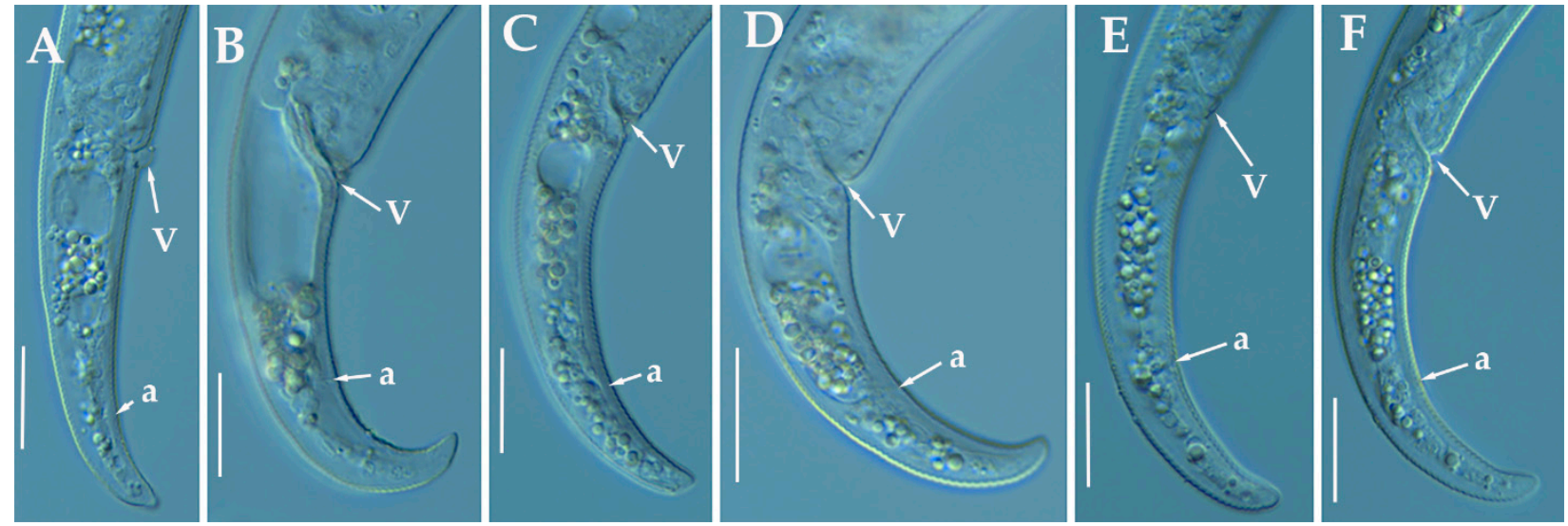

Figure 5. Light photomicrographs of Paratylenchus indalus sp. nov. female posterior regions (A-F). Scale bar: $20 \mu \mathrm{m})$. (Abbreviations: $\mathrm{a}=$ anus; $\mathrm{V}=$ vulva).

Male: Not found.

Juveniles: It is the most abundant developmental stage at the end of summer in the type locality and other localities. They were similar in morphology to females. However, they are characterized by the presence of a weak stylet; underdeveloped pharynx components; underdeveloped genital primordium; indistinct anus; and posterior body with a rounded or pointed terminus.

\section{Diagnosis and Relationships}

The new species can be characterized by the presence of 4 lateral lines, advulval flaps, and a stylet length of $28.3(26.0-29.5) \mu \mathrm{m}$. Lip region rounded, with the anterior end flattened, continuous with the rest of the body. Excretory pore situated at the level or anterior to the pharyngeal basal bulb. Spermatheca rounded. Tail conoid gradually tapering to form a rounded terminus.

Morphologically, the new species is close to P. projectus, P. neoprojectus Wu \& Hawn, 1975 and P. enigmaticus. From these species no important and clear morphological and morphometrical differences can be detected. However, P. projectus and P. neoprojectus have important differences molecularly to $P$. indalus sp. nov. Molecular differences using population paratypes from $P$. projectus and $P$. neoprojectus must be necessary in order to separate these three closely related morphologically species. Paratylenchus projectus differs from $P$. neoprojectus by having a trapezoid-shaped lip region, more anterior position of excretory pore and often digitate tail terminus, but these characters could have some variation [36]. These species are also similar to P. nanus and P. neoamblycephalus from which differ by having empty spermatheca and absence of males [36]. Molecularly, this species is closely related but different to paratypes from P. enigmaticus. This is a case of cryptic speciation among these three species comprising a complex group of pin nematodes.

\section{Molecular Characterization}

Ten D2-D3 of 28S rRNA (MW798273-MW798282), ten ITS (MW798319- MW798328), and four COI gene sequences (MW797005-MW797008) from the four different populations were generated herein for this species. All sequences showed no intraspecific variation, except for the ITS sequences, where one variable position was found. Paratylenchus indalus sp. nov. was closely related with P. enigmaticus, showing similarity values of $96 \%$ (differing by 25 nucleotides and 1 indel) for the D2-D3 region with several accession from P. enigmaticus, such as MN535546 or MW282761. For the ITS region, the similarity values were from 94 to $96 \%$ (differing by 24 to 39 nucleotides and from 10 to 15 indels) with the P. enigmaticus accessions MW282773 and MN535549, respectively. Finally, the similarity found for the COI gene sequences was $97 \%$ (differing by 11 nucleotides) with P. enigmaticus accessions 
(MW421686, MN782403 and MW316640). Paratylenchus indalus sp. nov. is not so closely molecularly related to $P$. projectus and $P$. neoprojectus as for $P$. enigmaticus, with a similarity of $92-93 \%, 89 \%$ and $87 \%$ for D2-D3 of $28 \mathrm{~S}$ rRNA, ITS region and COI, respectively.

Remarks

This species has been found in four almond orchards with undetermined rootstock in four different localities in Almería province (South-eastern Spain). The population presented moderate to high numbers of individuals in soil (from 68 to 2268 individuals $/ 500 \mathrm{~cm}^{3}$ of soil), being the majority of them $\mathrm{J} 4$ at the end of summer.

Type Habitat and Locality

Paratylenchus indalus sp. nov. was found in the rhizosphere of almond at Santa María de Nieva, Almería province (coordinates $37^{\circ} 35^{\prime} 26.8^{\prime \prime} \mathrm{N} ; 2^{\circ} 02^{\prime} 03.5^{\prime \prime} \mathrm{W}$ ), and also has been found in four localities in Almería province (Table 1).

Etymology

The species epithet, indalus, is derived from the name 'indalo' a prehistoric symbol found in a cave of Almería, the province of the locality where the type specimens were collected.

Type Material

Holotype female, and 11 paratypes females (slide numbers PR_104-01 to PR_104-06) were deposited in the Nematode Collection of the Institute for Sustainable Agriculture, CSIC, Córdoba, Spain, and two females deposited at the USDA Nematode Collection (slide T-7480p).

\subsubsection{Description of Paratylenchus pedrami sp. nov.}

(Figures 6-8; Table 4). http:/ / zoobank.org/NomenclaturalActs/1ABA6B7F-79FB4AAF-BD9D-8765183A3353 (accessed on 27 March 2021).

Female: Body slender, ventrally arcuate to form an open C-shaped when heat relaxed. Cuticle finely annulated; lateral field equidistant with four distinct lines. Lip region rounded, with anterior end flattened, continuous with the rest of the body, presence of small submedian lobes. Labial framework sclerotization weak; pharyngeal region typical paratylenchoid type. Stylet rigid, straight; stylet knobs rounded; dorsal pharyngeal gland opening 3.5-5.0 $\mu \mathrm{m}$ behind stylet knobs. Median pharyngeal bulb slender elongate, bearing distinct large valves; isthmus short slender, surrounded by nerve ring; basal bulb pyriform, pharyngeal-intestinal valve rounded; excretory pore situated at the level or anterior to pharyngeal basal bulb. Hemizonid 1-2 annuli long situated immediately anterior to excretory pore; body slightly narrower posterior to vulva; ovary outstretched, well developed. Spermatheca and crustaformeria well developed; spermatheca rounded; vulva a transverse slit occupying half of the corresponding body width; advulval lips prominent, the anterior lip is protruding further than the posterior lip; vulval flaps present, but not prominent in fixed specimens. Anus difficult to distinguish. Tail slender, conoid, finely annulated, and gradually tapering to form a rounded terminus or pointed in some individuals (Figure 8).

Male: Body slender than female, tapering towards both ends, cuticle apparently smooth with fine annulations; labial region similar to that of female but narrower and slightly truncated, continuous with body, sclerotization in labial region weak. Stylet lacking; pharynx rudimentary, procorpus, metacorpus, and basal bulb inconspicuous, and nonfunctional. Excretory pore located $65.5 \mu \mathrm{m}$ from anterior end. Testis outstretched, with small spermatozoa; spicule slender, slightly curved towards end; gubernaculum curved; bursa absent; tail short and rounded. 

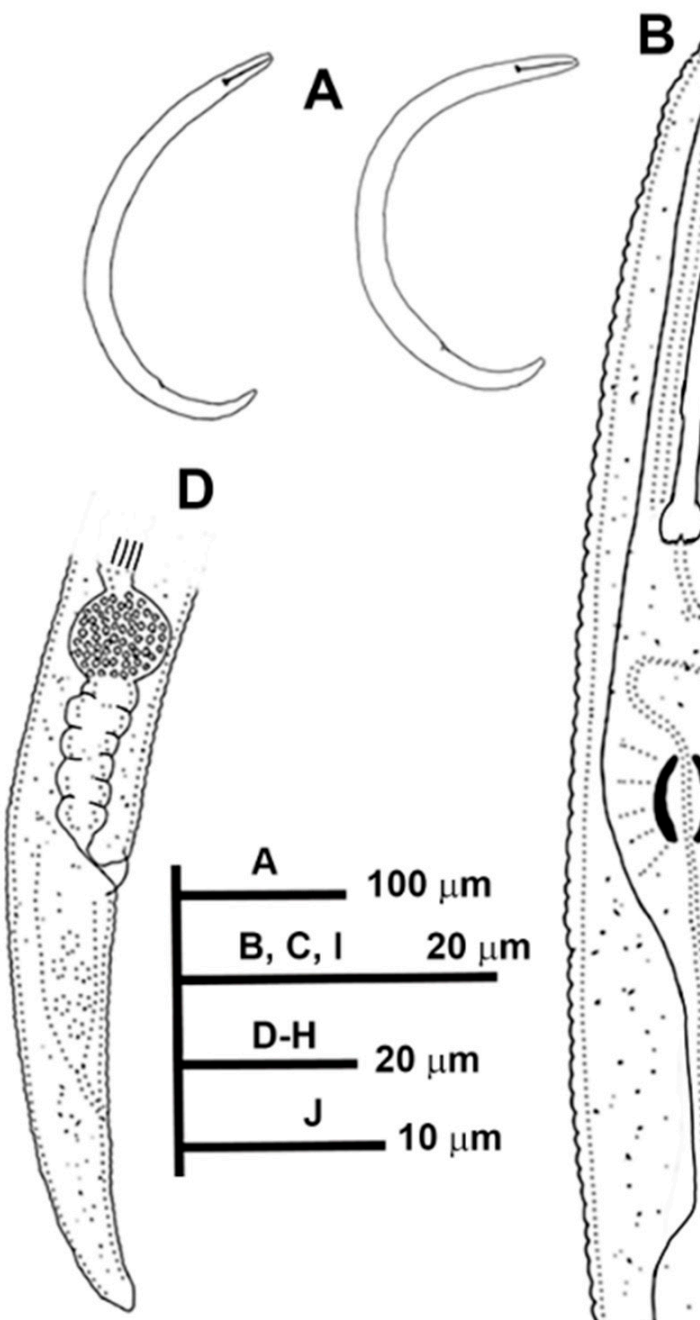

\section{$\mathbf{E}$}
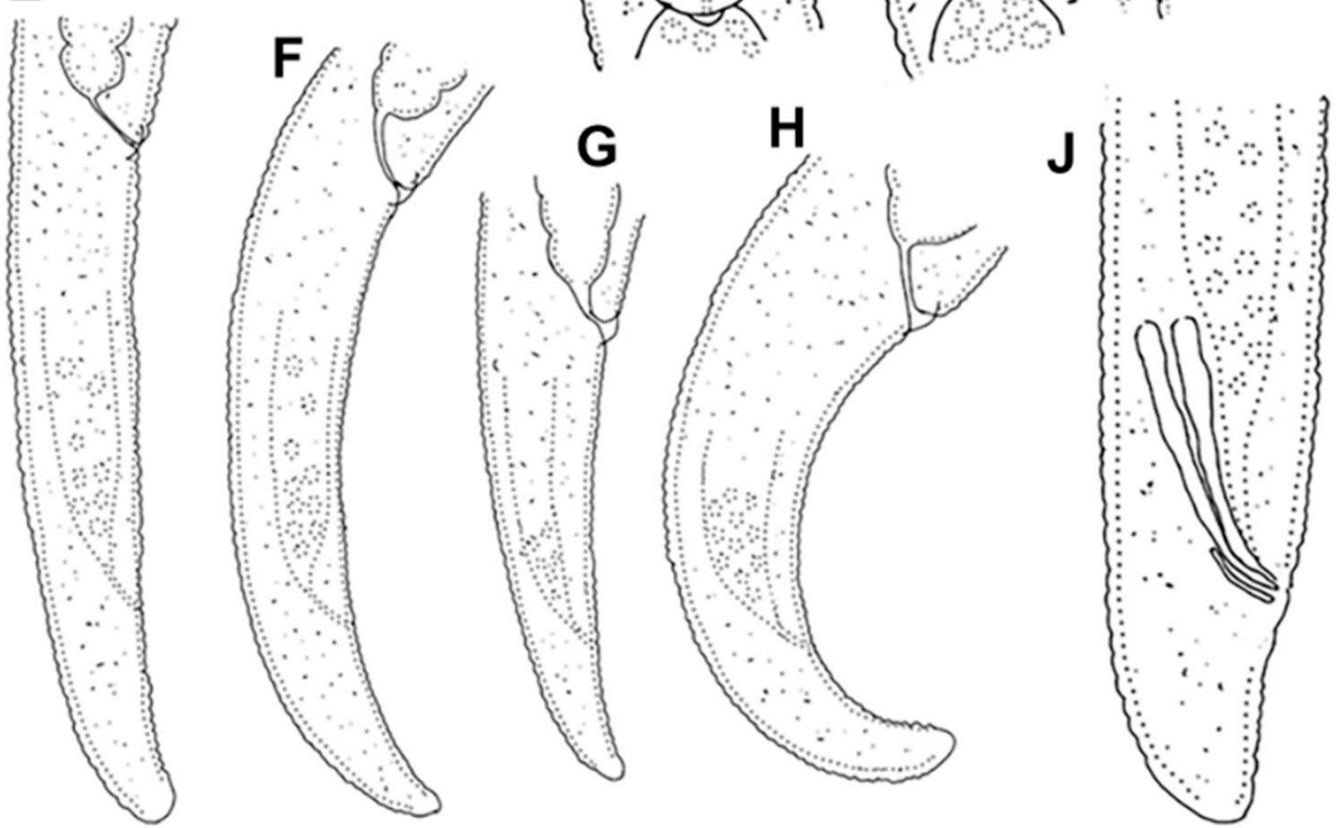

Figure 6. Line drawings of Paratylenchus pedrami sp. nov. (A): Entire females; (B,C): Female pharyngeal region; (D-H): Female posterior region; (I): Male pharyngeal region showing absence of stylet; (J): Male posterior region. 

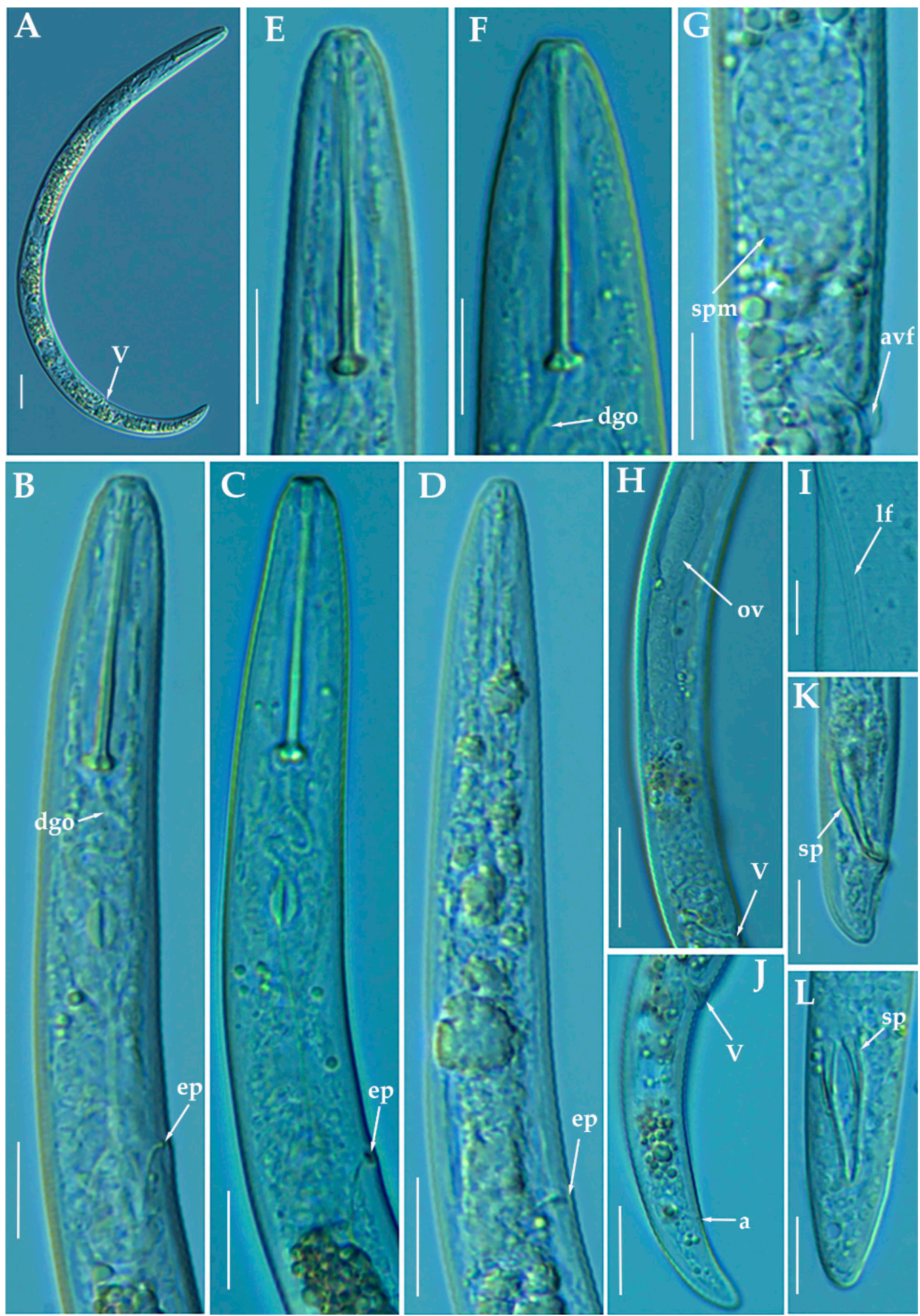

Figure 7. Light photomicrographs of female of Paratylenchus pedrami sp. nov. (A): Entire female with vulva arrowed; (B,C): Female pharyngeal region; (D): Male pharyngeal region; (E,F): Female lip region; (G): Vulval region showing spermatheca (arrowed); $(\mathbf{H})$ : Female posterior region showing complete genital branch; (I): Detail of lateral field at mid-body (arrowed); (J): Female posterior region with vulva and anus arrowed; $\mathrm{K}$, L: Male posterior region with spicules (arrowed). Scale bars $(\mathbf{A}, \mathbf{H}, \mathbf{J}=20 \mu \mathrm{m} ; \mathbf{B}-\mathbf{G}, \mathbf{I}, \mathbf{K}, \mathbf{L}=10 \mu \mathrm{m})$. (Abbreviations: a = anus; avf = advulval flap; dgo = pharyngeal dorsal gland orifice; ep = excretory pore; lf = lateral field; ov = ovary; $\mathrm{spm}$ = spermatheca; $\mathrm{V}=\mathrm{vulva}$ ). 

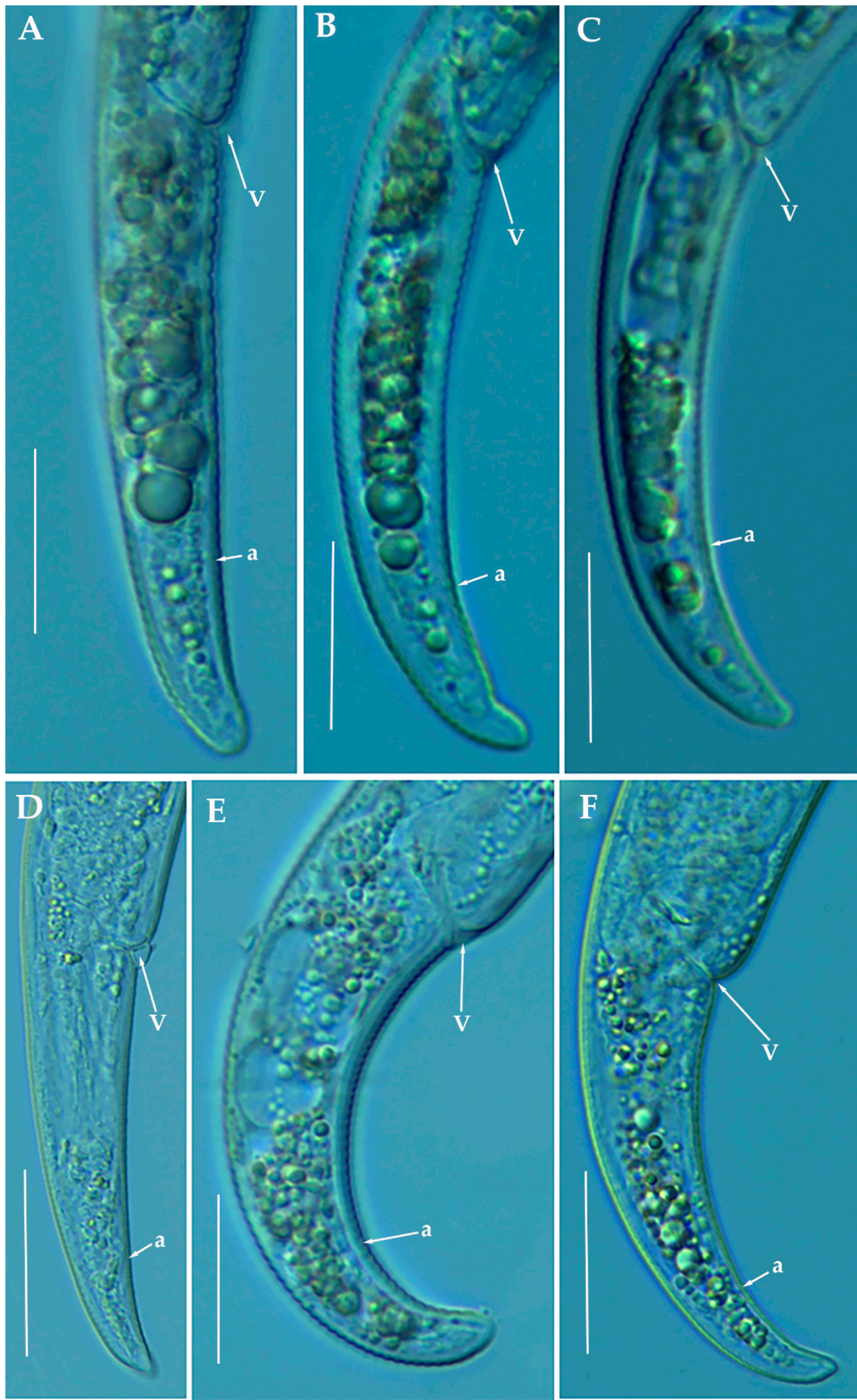

Figure 8. Light photomicrographs of Paratylenchus pedrami sp. nov. female posterior regions (A-F). Scale bars $(\mathbf{A}-\mathbf{F}=20 \mu \mathrm{m})$. (Abbreviations: $\mathrm{a}=$ anus; $\mathrm{V}=$ vulva). 
Table 4. Morphometrics of Paratylenchus pedrami sp. nov. paratype females, males and other population from Spain. All measurements are in $\mu \mathrm{m}$ and in the form: mean \pm s.d. (range).

\begin{tabular}{|c|c|c|c|c|}
\hline Measurements and Ratios & Holotype Female & $\begin{array}{l}\text { Paratype } \\
\text { Females }\end{array}$ & Paratype Males & Females \\
\hline Sample code & PR_14 & PR_14 & PR_14 & PR_17 \\
\hline $\begin{array}{l}\text { Locality } \\
n\end{array}$ & $\begin{array}{c}\text { Córdoba, Córdoba } \\
1\end{array}$ & $\begin{array}{c}\text { Córdoba, Córdoba } \\
20\end{array}$ & $\begin{array}{c}\text { Córdoba, Córdoba } \\
3\end{array}$ & $\begin{array}{c}\text { Córdoba, Córdoba } \\
4\end{array}$ \\
\hline $\mathrm{L}$ & 295 & $\begin{array}{l}297 \pm 30.0 \\
(231-374)\end{array}$ & $\begin{array}{c}269.7 \pm 21.6 \\
(245-285)\end{array}$ & $\begin{array}{l}305 \pm 7.7 \\
(294-312)\end{array}$ \\
\hline$a *$ & 21.1 & $\begin{array}{l}18.6 \pm 3.1 \\
(13.7-23.0)\end{array}$ & $\begin{array}{l}22.6 \pm 1.7 \\
(21.1-24.5)\end{array}$ & $\begin{array}{l}20.1 \pm 1.3 \\
(18.9-22.0)\end{array}$ \\
\hline $\mathrm{b}$ & 3.7 & $\begin{array}{l}3.7 \pm 0.3 \\
(3.2-4.5)\end{array}$ & $\begin{array}{l}4.5 \pm 0.4 \\
(4.0-4.8)\end{array}$ & $\begin{array}{l}3.6 \pm 0.1 \\
(3.5-3.7)\end{array}$ \\
\hline c & 13.1 & $\begin{array}{l}13.0 \pm 1.9 \\
(10.0-16.5)\end{array}$ & $\begin{array}{l}24.2 \pm 1.8 \\
(22.3-25.9)\end{array}$ & $\begin{array}{l}8.6 \pm 0.6 \\
(8.2-9.6)\end{array}$ \\
\hline$c^{\prime}$ & 2.6 & $\begin{array}{l}2.8 \pm 0.1 \\
(2.6-3.1)\end{array}$ & $\begin{array}{l}1.4 \pm 0.1 \\
(1.3-1.6)\end{array}$ & $\begin{array}{l}3.5 \pm 0.1 \\
(3.4-3.7)\end{array}$ \\
\hline $\mathrm{V}$ or $\mathrm{T}$ & 81.4 & $\begin{array}{l}79.9 \pm 1.6 \\
(76.0-82.0)\end{array}$ & $\begin{array}{l}34.7 \pm 4.6 \\
(30.1-39.3)\end{array}$ & $\begin{array}{l}81.2 \pm 1.0 \\
(80.1-82.3)\end{array}$ \\
\hline G1 & 34.9 & $\begin{array}{l}35.8 \pm 6.1 \\
(26.2-50.0)\end{array}$ & - & $\begin{array}{l}34.4 \pm 7.8 \\
(28.8-39.9)\end{array}$ \\
\hline Stylet length & 28.0 & $\begin{array}{l}27.8 \pm 1.1 \\
(26.0-30.0)\end{array}$ & - & $\begin{array}{l}30.9 \pm 1.0 \\
(29.5-32.0)\end{array}$ \\
\hline Conus length & 22.0 & $\begin{array}{l}18.5 \pm 1.2 \\
(16.0-20.5)\end{array}$ & - & $\begin{array}{l}22.0 \pm 1.6 \\
(20.0-24.0)\end{array}$ \\
\hline $\mathrm{m}$ & 78.6 & $\begin{array}{l}66.4 \pm 2.8 \\
(61.5-70.4)\end{array}$ & - & $\begin{array}{l}71.2 \pm 3.0 \\
(67.8-75.0)\end{array}$ \\
\hline DGO & 4.0 & $\begin{array}{l}3.9 \pm 0.3 \\
(3.5-5.0)\end{array}$ & - & $\begin{array}{l}4.6 \pm 0.5 \\
(4.0-5.0)\end{array}$ \\
\hline $\mathrm{O}$ & 14.3 & $\begin{array}{l}14.2 \pm 1.4 \\
(12.3-19.2)\end{array}$ & - & $\begin{array}{l}15.0 \pm 1.7 \\
(12.5-16.1)\end{array}$ \\
\hline Lip width & 4.5 & $\begin{array}{l}3.8 \pm 0.5 \\
(3.0-5.0)\end{array}$ & $3.0 \pm 0.0$ & $\begin{array}{l}5.5 \pm 0.4 \\
(5.0-6.0)\end{array}$ \\
\hline Median bulb length & 16.5 & $\begin{array}{l}19.2 \pm 1.4 \\
(17.0-22.0)\end{array}$ & - & $\begin{array}{l}19.4 \pm 1.3 \\
(18.0-21.0)\end{array}$ \\
\hline Median bulb width & 7.0 & $\begin{array}{l}8.5 \pm 0.4 \\
(8.0-9.0)\end{array}$ & - & $\begin{array}{l}8.1 \pm 0.9 \\
(7.5-9.5)\end{array}$ \\
\hline $\begin{array}{l}\text { Anterior end to center median } \\
\text { bulb }\end{array}$ & 45.0 & $\begin{array}{l}46.0 \pm 2.8 \\
(39.0-50.0)\end{array}$ & - & $49.0 \pm 0.0$ \\
\hline - & 57.0 & $\begin{array}{l}57.0 \pm 2.9 \\
(53.2-65.3)\end{array}$ & - & $\begin{array}{l}57.8 \pm 0.3 \\
(57.6-58.3)\end{array}$ \\
\hline Nerve ring to anterior end & 60.0 & $\begin{array}{l}59.6 \pm 3.5 \\
(53.0-65.0)\end{array}$ & - & $\begin{array}{l}66.3 \pm 1.5 \\
(65.0-68.0)\end{array}$ \\
\hline Excretory pore to anterior end & 69.0 & $\begin{array}{l}71.6 \pm 5.3 \\
(62.0-80.0)\end{array}$ & 65.5 & $\begin{array}{l}77.8 \pm 3.5 \\
(74.0-82.0)\end{array}$ \\
\hline Pharynx length & 79.0 & $\begin{array}{l}80.1 \pm 4.6 \\
(70.0-86.0)\end{array}$ & $\begin{array}{c}60.3 \pm 9.7 \\
(52.0-71.0)\end{array}$ & $\begin{array}{l}84.8 \pm 0.5 \\
(84.0-85.0)\end{array}$ \\
\hline Maximum body diam. & 14.0 & $\begin{array}{c}16.5 \pm 3.3 \\
(12.0-23.0)\end{array}$ & $\begin{array}{c}12.0 \pm 1.8 \\
(10.0-13.5)\end{array}$ & $\begin{array}{c}15.3 \pm 1.0 \\
(14.0-16.5)\end{array}$ \\
\hline Tail length & 22.5 & $\begin{array}{c}23.2 \pm 3.1 \\
(18.0-28.0)\end{array}$ & $\begin{array}{c}11.2 \pm 1.3 \\
(10.0-12.5)\end{array}$ & $\begin{array}{l}35.5 \pm 2.6 \\
(32.0-38.0)\end{array}$ \\
\hline Anal body diam. & 8.5 & $\begin{array}{l}8.3 \pm 0.9 \\
(7.0-9.5)\end{array}$ & $\begin{array}{l}7.8 \pm 1.0 \\
(7.0-9.0)\end{array}$ & $\begin{array}{l}10.1 \pm 0.6 \\
(9.5-11.0)\end{array}$ \\
\hline Spicules & - & - & $\begin{array}{c}16.2 \pm 2.3 \\
(14.0-18.5)\end{array}$ & - \\
\hline Gubernaculum & - & - & $\begin{array}{l}3.5 \pm 0.5 \\
(3.0-4.0)\end{array}$ & - \\
\hline
\end{tabular}

* Abbreviations: $\mathrm{a}=$ body length/greatest body diameter; $\mathrm{b}=$ body length/distance from anterior end to pharyngo-intestinal junction; $\mathrm{DGO}=$ distance between stylet base and orifice of dorsal pharyngeal gland; $\mathrm{c}=$ body length $/$ tail length; $\mathrm{c}^{\prime}=$ tail length $/$ tail diameter at anus or cloaca; $\mathrm{G1}=$ anterior genital branch length expressed as percentage $(\%)$ of the body length; $\mathrm{L}=$ overall body length; $\mathrm{m}=$ length of conus as percentage of total stylet length; $\mathrm{MB}=$ distance between anterior end of body and center of median pharyngeal bulb expressed as percentage $(\%)$ of the pharynx length; $\mathrm{n}=$ number of specimens on which measurements are based; $\mathrm{O}=\mathrm{DGO}$ as percentage of stylet length; $\mathrm{T}=$ distance from cloacal aperture to anterior end of testis expressed as percentage $(\%)$ of the body length; $\mathrm{V}=$ distance from body anterior end to vulva expressed as percentage (\%) of the body length.

Juveniles: It is the most abundant developmental stage at the end of summer in the type locality and other localities. They were similar in morphology to adult females. 
However, they are characterized by the presence of weak stylet; underdeveloped pharynx components; underdeveloped genital primordium; indistinct anus; and posterior body with a rounded or pointed terminus.

Diagnosis and Relationships

The new species can be characterized by the presence of 4 lateral lines, advulval flaps, and a stylet length of $28.3(26.0-29.5) \mu \mathrm{m}$. Lip region rounded, with the anterior end flattened, continuous with the rest of the body. Excretory pore situated at the level or anterior to the pharyngeal basal bulb. Spermatheca rounded. Tail conoid gradually tapering to form a rounded terminus. Males with a short and rounded tail. According to species grouping by Ghaderi et al. [36] belongs to group 3 characterized by stylet length less than $40 \mu \mathrm{m}$, four lateral lines and advulval flaps present.

Morphologically, the new species is close morphologically and morphometrically to $P$. baldaccii, P. salubris, P. coronatus and P. mimulus. Paratylenchus pedrami sp. nov. differs from $P$. baldaccii in shape of the male tail, short and rounded vs. conical and pointed, and absence vs. presence of male stylet, slightly shorter body of females $(231-374 \mu \mathrm{m}$ vs. $280-430 \mu \mathrm{m})$ and different molecular markers studied in this article. A single male specimen of $P$. pedrami sp. nov. was sequenced for confirming these morphological differences with $P$. baldaccii. Paratylenchus pedrami sp. nov. differs from P. salubris in shorter female stylet (26.0-30.0 vs. $28.0-35.0 \mu \mathrm{m}$ ) and male tail shape, short and rounded vs. conical and pointed. Paratylenchus pedrami sp. nov. differs from P. coronatus in shorter female stylet (26.0-30.0 $\mu \mathrm{m}$ vs.31-39 $\mu \mathrm{m}$ ), in male tail shape (rounded vs. conoid-arcuate), and different molecular markers deposited in GenBank. Paratylenchus pedrami sp. nov. differs from P. mimulus in longer body of females (231-374 $\mu \mathrm{m}$ vs. $180-260 \mu \mathrm{m})$ and different male tail shape (rounded vs. conoid with finely rounded terminus).

\section{Molecular Characterization}

Two populations from this new species were molecularly characterized, including three identical D2-D3 of 28S rRNA (MW798283-MW798285), two identical ITS sequences (MW798329-MW798330) and one COI gene sequences (MW797009). The closest Paratylenchus sequences were from P. baldaccii with $96 \%, 93 \%$ and $90 \%$ similarity (differing by 30 , 45 and 26 nucleotides) for the D2-D3 of 28S, ITS region, and COI gene, respectively.

\section{Remarks}

This species has been found in two almond orchards with undetermined rootstock in two different localities in Córdoba province (Southern Spain). The population presented moderate numbers of individuals in soil (200 and 216 individuals $/ 500 \mathrm{~cm}^{3}$ of soil), being the majority of them $\mathrm{J} 4$ at the end of summer.

Paratylenchus pedrami sp. nov. was found in the rhizosphere of almond at Córdoba, Córdoba province (coordinates $37^{\circ} 49^{\prime} 39.9^{\prime \prime} \mathrm{N} ; 4^{\circ} 53^{\prime} 22.0^{\prime \prime} \mathrm{W}$ ), and also has been found in other place at the same locality (Table 1), both separated by approximately two kilometers.

Etymology

The species epithet, pedrami, is dedicated to Dr. Majid Pedram, an important Iranian nematologist from Department of Plant Pathology, Faculty of Agriculture, Tarbiat Modares University, Tehran, Iran.

\section{Type Material}

Holotype female, and 18 paratypes females (slide numbers PR_014-01 to PR_014-09) were deposited in the Nematode Collection of the Institute for Sustainable Agriculture, CSIC, Córdoba, Spain, and two females deposited at the USDA Nematode Collection (slide T-7481p). 
3.1.4. Description of Paratylenchus zurgenerus sp. nov.

(Figures 9-11; Table 5). http:/ / zoobank.org/NomenclaturalActs / 6BE06C25-B8CB-40 57-B2DE-3118A314073D (accessed on 27 March 2021).
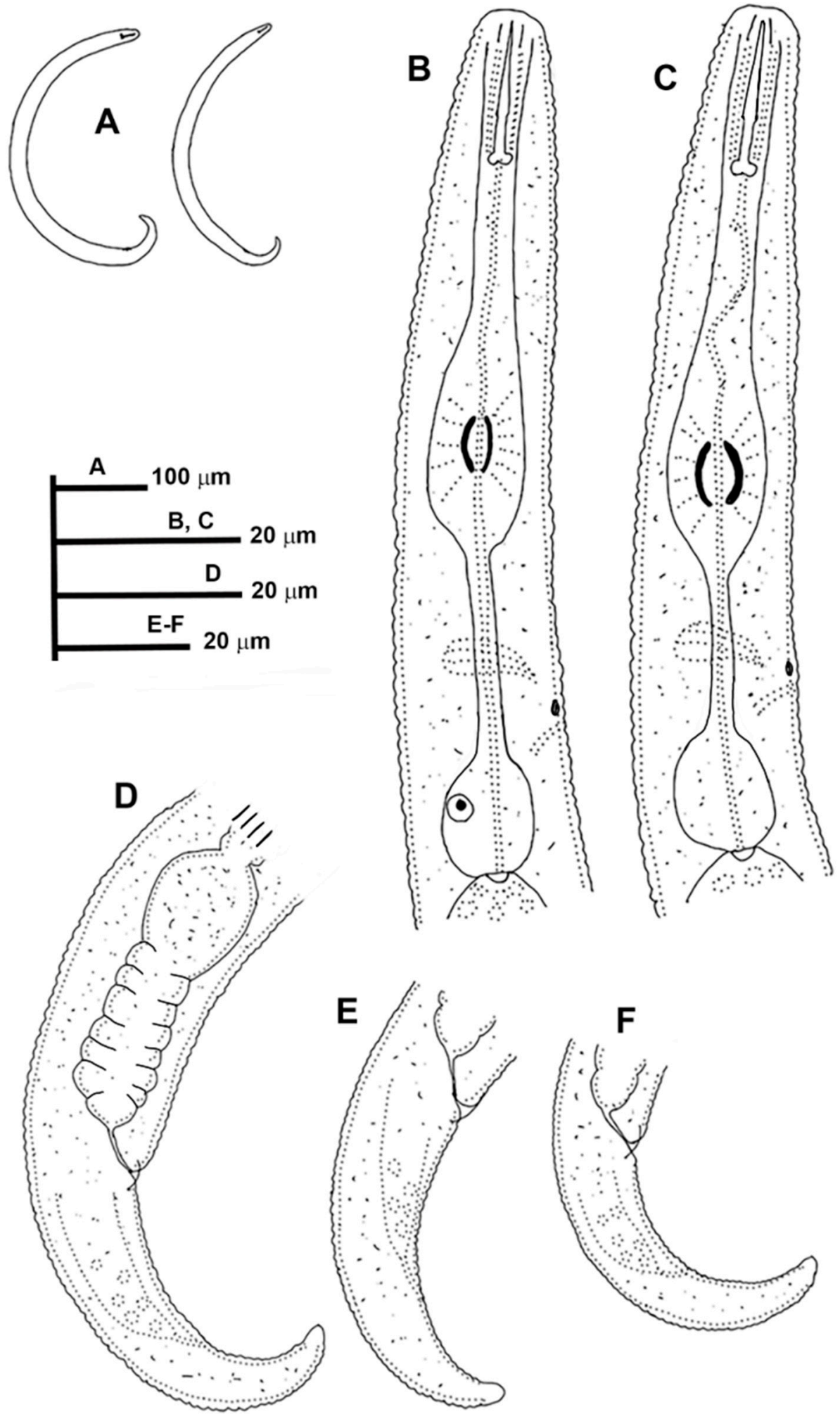

Figure 9. Line drawings of Paratylenchus zurgenerus sp. nov. (A): Entire females; (B,C): Female pharyngeal region; (D-F): Female posterior region. 

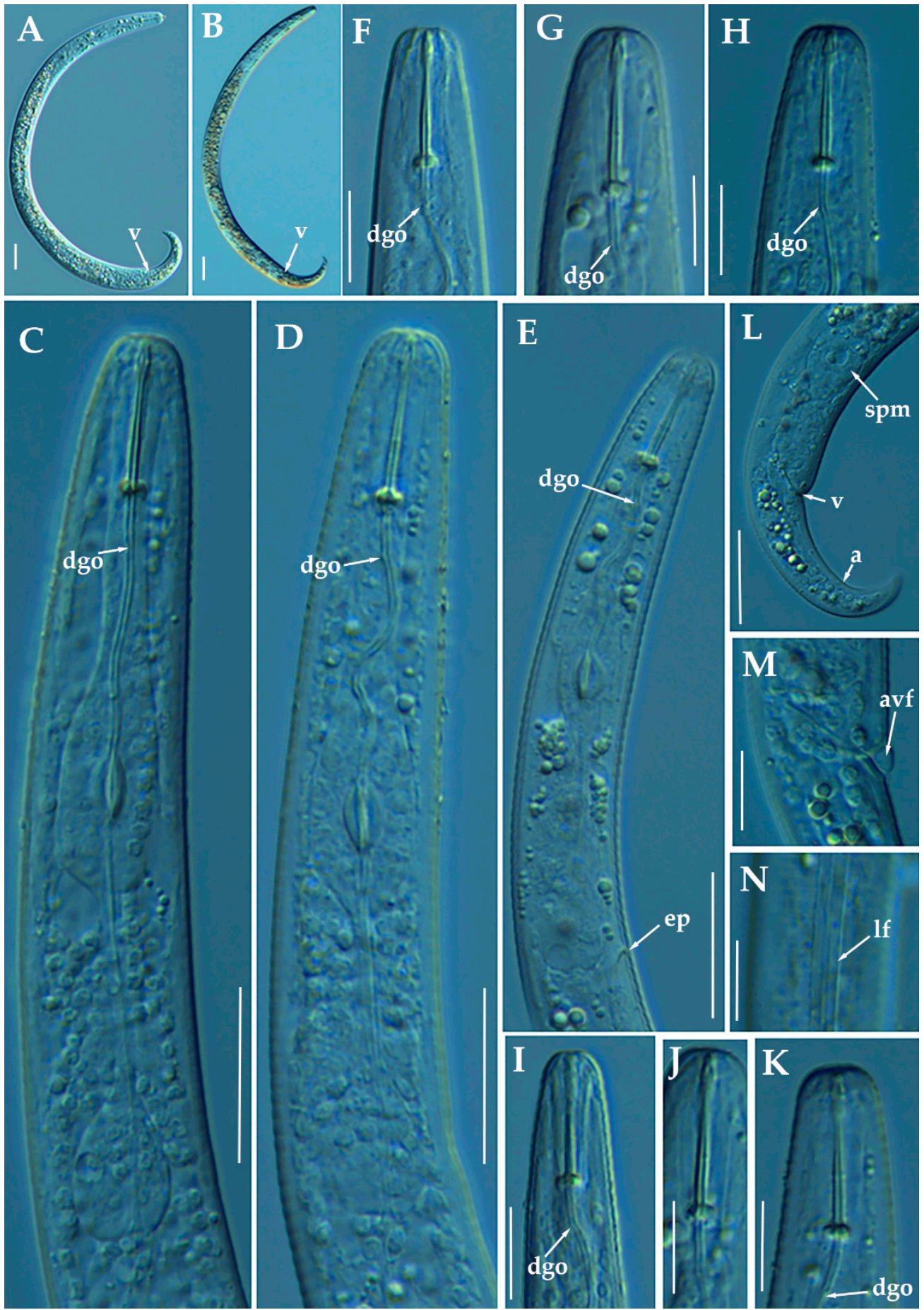

Figure 10. Light photomicrographs of female of Paratylenchus zurgenerus sp. nov. (A,B): Entire female with vulva arrowed; (C-E): Female pharyngeal region; (F-K): Female lip region; $(\mathbf{L})$ : Female posterior region showing empty spermatheca, vulva and anus (arrowed); (M): Detail of vulva showing advulval flap (arrowed); (N): Female mid-region with lateral field (arrowed). Scale bars $(\mathbf{A}-\mathbf{E}, \mathbf{L}=20 \mu \mathrm{m} ; \mathbf{F}-\mathbf{K}, \mathbf{M}, \mathbf{N}=10 \mu \mathrm{m})$. (Abbreviations: a = anus; avf = advulval flap; dgo = pharyngeal dorsal gland orifice; ep = excretory pore; lf = lateral field; $\mathrm{spm}=$ spermatheca; $\mathrm{V}=$ vulva). 

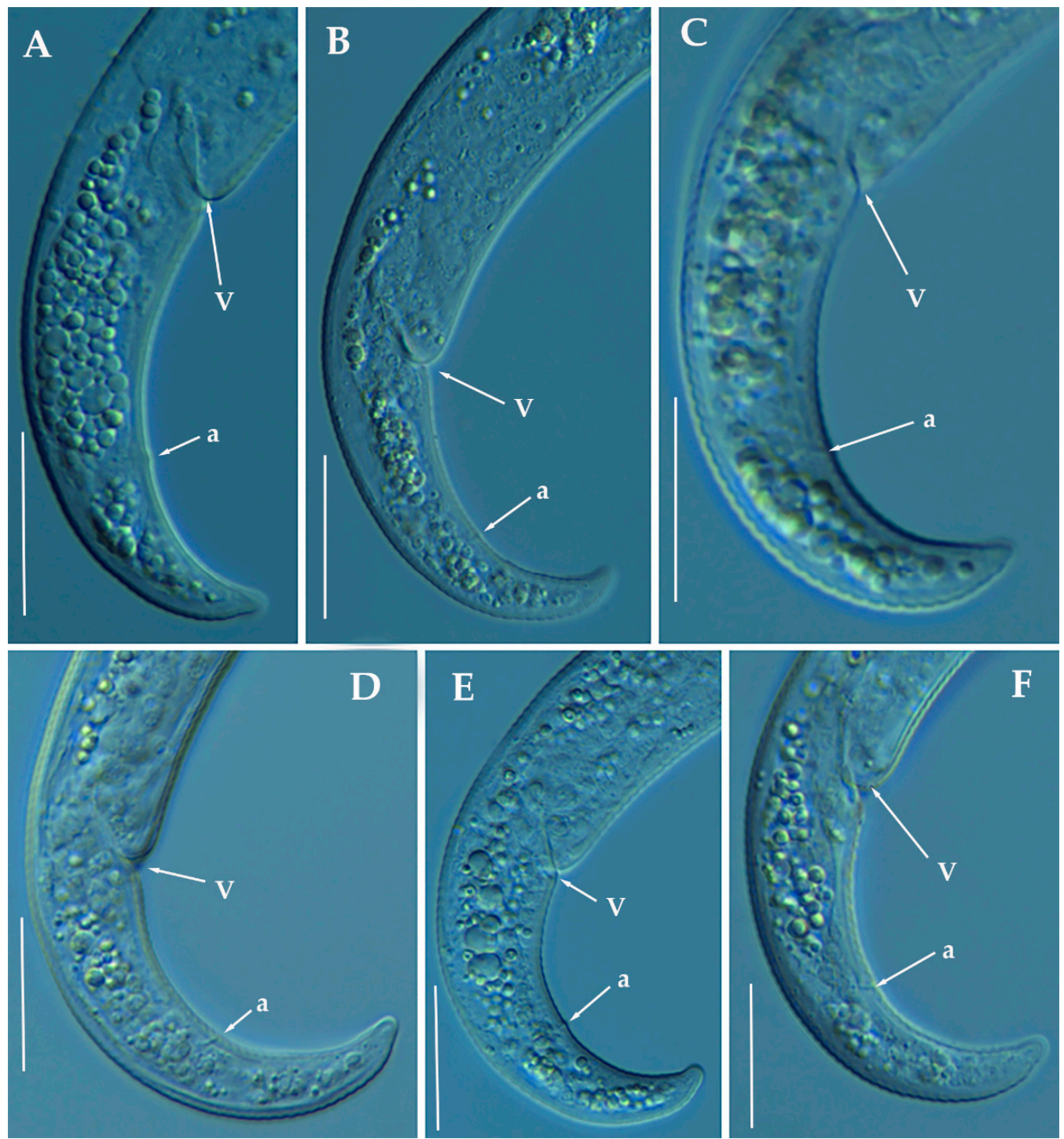

Figure 11. Light photomicrographs of Paratylenchus zurgenerus sp. nov. female posterior regions (A-F). Scale bars $(\mathbf{A}-\mathbf{F}=20 \mu \mathrm{m})$. (Abbreviations: $\mathrm{a}=$ anus; $\mathrm{V}=$ vulva).

Female: Body slender, ventrally arcuate to form a C-shaped when heat relaxed. Cuticle finely annulated; lateral field equidistant with four distinct lines. Lip region rounded, with anterior end flattened, continuous with the rest of the body, absence of submedian lobes. Labial framework sclerotization relatively strong; pharyngeal region typical paratylenchoid type. Stylet delicate, straight; stylet knobs rounded; dorsal pharyngeal gland opening 3.5-5.0 $\mu \mathrm{m}$ behind stylet knobs. Median pharyngeal bulb slender elongate, bearing distinct large valves; isthmus short slender, surrounded by nerve ring; basal bulb pyriform, pharyngeal-intestinal valve rounded. Excretory pore situated at the level or anterior to pharyngeal basal bulb; hemizonid 1-2 annuli long situated immediately anterior to excretory pore; body slightly narrower posterior to vulva. Ovary outstretched, well developed; spermatheca and crustaformeria well developed; spermatheca rounded; vulva a transverse slit occupying half of the corresponding body width; vulval lips prominent, the anterior lip is protruding further than the posterior lip; vulval flaps present, but not prominent in fresh specimens. Anus difficult to distinguish (more distinguishable in alive specimens). Tail slender, conoid, finely annulated, and gradually tapering to form a rounded terminus or pointed in some individuals (Figure 11). 
Table 5. Morphometrics of Paratylenchus zurgenerus sp. nov. paratype females from Zurgena, Almería province (Spain). All measurements are in $\mu \mathrm{m}$ and in the form: mean \pm s.d. (range).

\begin{tabular}{|c|c|c|}
\hline Measurements and Ratios & Holotype Female & Paratype Females \\
\hline Sample code & PR_111 & PR_111 \\
\hline $\mathrm{n}$ & 1 & 19 \\
\hline $\mathrm{L}$ & 370 & $356.9 \pm 32.1(316-418)$ \\
\hline$a^{*}$ & 20.0 & $20.1 \pm 1.7(17.5-23.7)$ \\
\hline $\mathrm{b}$ & 3.7 & $3.9 \pm 0.4(3.4-4.9)$ \\
\hline c & 11.9 & $13.8 \pm 1.7(11.0-17.0)$ \\
\hline$c^{\prime}$ & 2.8 & $2.7 \pm 0.3(2.0-3.2)$ \\
\hline $\mathrm{V}$ & 83.2 & $84.8 \pm 1.0(83.3-86.6)$ \\
\hline G1 & 40.0 & $28.4 \pm 6.9(18.2-43.5)$ \\
\hline Stylet length & 15.0 & $15.4 \pm 0.6(14.0-16.0)$ \\
\hline Conus length & 9.0 & $9.0 \pm 0.6(8.0-10.0)$ \\
\hline $\mathrm{m}$ & 60.0 & $58.6 \pm 3.4(53.1-66.7)$ \\
\hline DGO & 5.5 & $5.0 \pm 0.8(3.5-6.0)$ \\
\hline $\mathrm{O}$ & 36.7 & $32.4 \pm 4.4(22.6-37.5)$ \\
\hline Lip width & 6.0 & $6.7 \pm 0.6(6.0-8.0)$ \\
\hline Median bulb length & - & $22.1 \pm 1.96(19.0-26.0)$ \\
\hline Median bulb width & - & $10.0 \pm 0.9(9.0-12.0)$ \\
\hline Anterior end to center median bulb & 55.0 & $47.7 \pm 3.0(41.0-52.0)$ \\
\hline $\mathrm{MB}$ & 55.3 & $51.6 \pm 2.1(47.2-55.1)$ \\
\hline Nerve ring to anterior end & 70.0 & $64.9 \pm 4.2(59.0-73.0)$ \\
\hline Excretory pore to anterior end & 83.0 & $81.3 \pm 7.4(67.0-94.0)$ \\
\hline Pharynx length & 99.5 & $92.5 \pm 4.5(85.0-101.0)$ \\
\hline Maximum body diam. & 18.5 & $17.8 \pm 2.0(15.5-22.0)$ \\
\hline Tail length & 31.0 & $26.2 \pm 3.5(22.0-34.5)$ \\
\hline Anal body diam. & 11.0 & $9.7 \pm 1.1(8.0-11.5)$ \\
\hline
\end{tabular}

* Abbreviations: $\mathrm{a}=$ body length/greatest body diameter; $\mathrm{b}=$ body length/distance from anterior end to pharyngo-intestinal junction; DGO = distance between stylet base and orifice of dorsal pharyngeal gland; $\mathrm{c}=$ body length/tail length; $c^{\prime}=$ tail length/tail diameter at anus or cloaca; G1 = anterior genital branch length expressed as percentage (\%) of the body length; $\mathrm{L}=$ overall body length; $\mathrm{m}=$ length of conus as percentage of total stylet length; $\mathrm{MB}=$ distance between anterior end of body and center of median pharyngeal bulb expressed as percentage $(\%)$ of the pharynx length; $\mathrm{n}=$ number of specimens on which measurements are based; $\mathrm{O}=\mathrm{DGO}$ as percentage of stylet length; $\mathrm{V}=$ distance from body anterior end to vulva expressed as percentage (\%) of the body length.

Male: Not found.

Juveniles: It is the most abundant developmental stage at the end of summer in the type locality. Fourth-life stage of individuals was similar in morphology to the adult females. However, they are characterized by the presence of a weak stylet $(10-11 \mu \mathrm{m})$; underdeveloped pharynx components; underdeveloped genital primordium; indistinct anus; and posterior body with a rounded or pointed terminus.

\section{Diagnosis and Relationships}

The new species can be characterized by the presence of 4 lateral lines, advulval flaps, and a short and delicate stylet length of $15.4(14.0-16.0) \mu \mathrm{m}$. Lip region rounded, with the anterior end flattened, continuous with the rest of the body. Excretory pore situated at the level or anterior to the pharyngeal basal bulb. Spermatheca rounded. Tail conoid gradually tapering to form a rounded terminus. According to species grouping by Ghaderi et al. [36] belongs to group 3 characterized by stylet length less than $40 \mu \mathrm{m}$, four lateral lines and advulval flaps present.

Morphologically, the new species is close to P. microdorus, Paratylenchus recisus Siddiqi, 1996, and P. veruculatus. Paratylenchus zurgenerus sp. nov. differs from the original type population of $P$. microdorus in a posterior position of the vulva $(83.3-86.6 \%$ vs. $81-82 \%)$, smaller c' ratio (2.0-3.2 vs. 4.5), and posterior position of excretory pore (67.0-94.0 $\mu \mathrm{m}$ vs. $65 \mu \mathrm{m})$. Tail in P. microdorus is variable, pointed or rounded, and specimens with various tail ends have been found in almost every population examined [36], but our populations showed a prominent rounded tip. Molecular data of P. microdorus [24] clearly 
differs from our population of P. zurgenerus sp. nov. (see below). Paratylenchus zurgenerus sp. nov. differs from the original type population of P. recisus in longer body $(316-418 \mu \mathrm{m}$ vs. $270-390 \mu \mathrm{m})$, posterior position of the vulva $(83.3-86.6 \%$ vs. $78-83 \%)$, and wider lip region $(6.0-8.0 \mu \mathrm{m}$ vs. $5.0-5.2 \mu \mathrm{m})$.

\section{Molecular Characterization}

Four D2-D3 of 28S rRNA (MW798286-MW798289), and four ITS sequences (MW798331MW798334) were obtained for this species. In both ribosomal genes, no intraspecific variability was detected between three of the four individuals sequenced, whereas in the fourth one, 11 different nucleotides were detected for the D2-D3 (MW798289) and 24 nucleotides and 4 indels for the ITS region (MW798334). Two identical COI gene sequences (MW797010MW797011) were generated for P. zurgenerus sp. nov. The D2-D3 sequences were found to be $99 \%$ similar (differing from 1 to 11 nucleotides) and in the same phylogenetic clade of Paratylenchus sp.7 (KF242242) from California (USA) that should be consider as conspecific to $P$. zurgenerus sp. nov., however, only molecular and no morphological data are available for this species [23]. The closest Paratylenchus spp. for the rest of the molecular markers studied was P. microdorus (MW413599-MW413600) being 87\% similar for the ITS region (differing by 38 to 41 nucleotides and from 28 to 31 indels) and $91 \%$ for COI sequences (MW421666-MW421667) (differing by 28 nucleotides and no indels).

Remarks

This species has been found in one almond orchard with undetermined rootstock in one locality in Granada province (Southern Spain). The population presented moderatehigh numbers of individuals in soil (1470 individuals $/ 500 \mathrm{~cm}^{3}$ of soil), being the majority of them J4 at the end of summer. Probably this species could be the same previously described by Gomez-Barcina et al. [45] as P. microdorus as its measurements are identical and in geographically related areas in Southern Spain, but further studies are needed for confirming this hypothesis.

Type Habitat and Locality

Paratylenchus zurgenerus sp. nov. was only found in the rhizosphere of almond at Zurgena, Almería province (coordinates $37^{\circ} 24^{\prime} 22.5^{\prime \prime} \mathrm{N} 2^{\circ} 02^{\prime} 00.3^{\prime \prime} \mathrm{W}$ ) (Table 1 ).

Etymology

The species epithet, zurgenerus, refers to the name of the gentilice of inhabitants of the type locality (Zurgena).

Type Material

Holotype female, and 17 paratypes females (slide numbers PR_111-01 to PR_111-09) were deposited in the Nematode Collection of the Institute for Sustainable Agriculture, CSIC, Córdoba, Spain, and two females deposited at the USDA Nematode Collection (slide T-7482p).

\subsubsection{Morphometrics and Remarks of Known Paratylenchus Spanish Populations}

Morphometrical data as well as molecular characterization of other already known Paratylenchus populations detected in the present study were compared with original and previous reported populations, and these species included $P$. baldaccii, $P$. enigmaticus, $P$. goodeyi, P. hamatus, P. holdemani, P. israelensis, P. tenuicaudatus, and P. veruculatus.

Paratylenchus enigmaticus Munawar, Yevtushenko, Palomares-Rius \& Castillo, 2021.

This species has been recently described from Canada [21]; and it has also been reported in Belgium [22,24]. In the present study, the P. enigmaticus population from Spain matches with the original species description, except for minor differences in body length and a ratio; the Spanish population is slightly shorter than the original one (324-383 $\mu \mathrm{m}$ vs. $343-431 \mu \mathrm{m})$ and with smaller a ratio (17.6-21.6 vs. 21.7-28.7) (Table 6, Figure 12). 
This population is closely related morphometrically to the T1-T5 populations found in Belgium [22]. This species has not been reported before in Spain and constitutes the first record for the country.
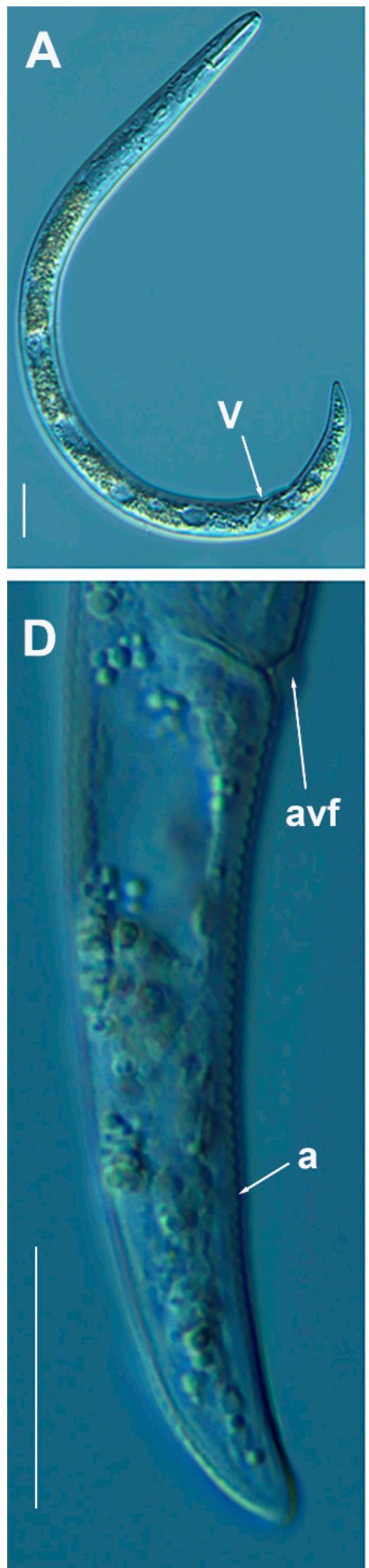

\section{$\mathbf{B}$}
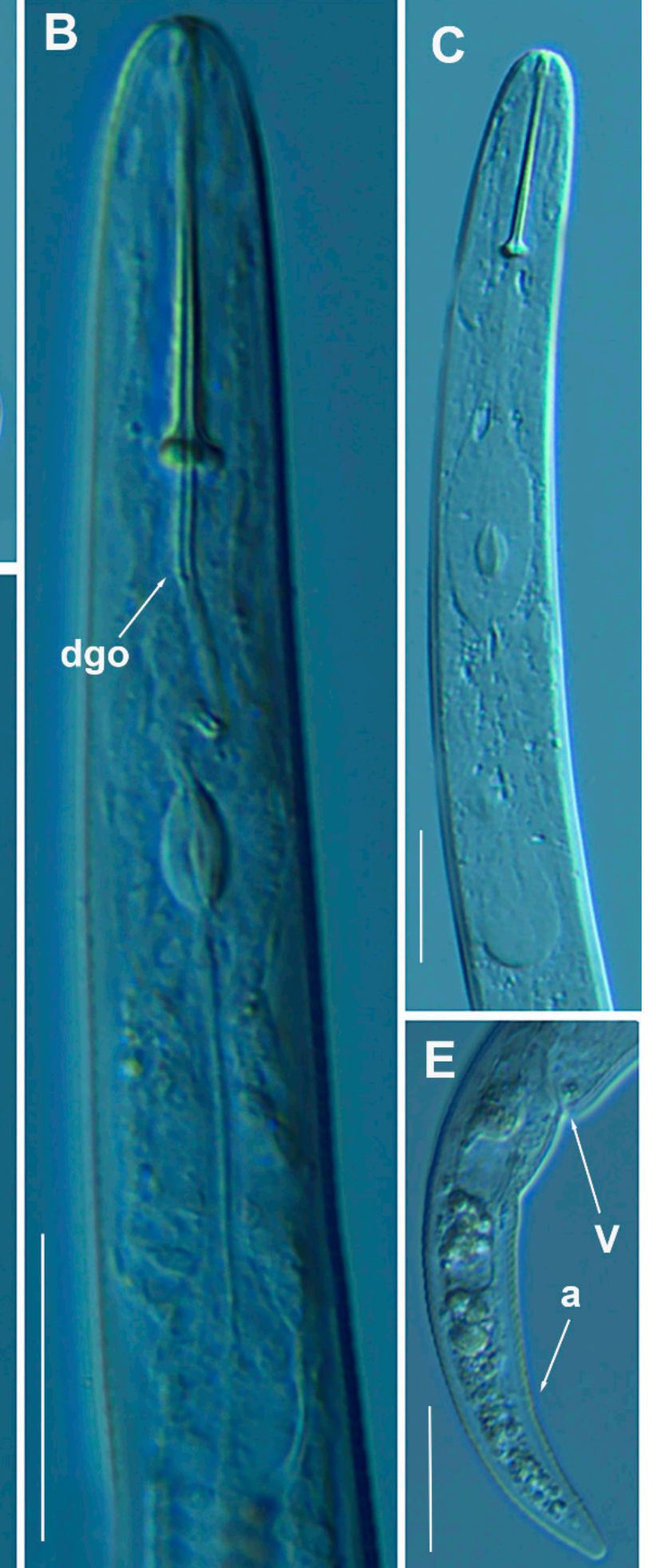

Figure 12. Light photomicrographs of Paratylenchus enigmaticus Munawar, Yevtushenko, PalomaresRius \& Castillo, 2021. (A): Entire female with vulva arrowed; (B,C): Female pharyngeal region; (D,E): Female posterior region showing vulva and anus (arrowed). Scale bars $(\mathbf{A}-\mathbf{E}=20 \mu \mathrm{m})$. (Abbreviations: $\mathrm{a}=$ anus; $\mathrm{avf}=$ advulval flap; $\mathrm{dgo}=$ pharyngeal dorsal gland orifice; $\mathrm{V}=$ vulva). 
Table 6. Morphometrics of Paratylenchus baldaccii Raski, 1975, Paratylenchus enigmaticus Munawar, Yevtushenko, PalomaresRius \& Castillo, 2021, Paratylenchus goodeyi (Oostenbrink, 1953) Raski, 1962, Paratylenchus holdemani Raski, 1975 and Paratylenchus israelensis (Raski, 1973) Siddiqi, 1986 from several localities in Spain. All measurements are in $\mu \mathrm{m}$ and in the form: mean \pm s.d. (range).

\begin{tabular}{|c|c|c|c|c|c|c|c|}
\hline $\begin{array}{l}\text { Measurements } \\
\text { and Ratios }\end{array}$ & \multirow{2}{*}{$\begin{array}{c}\text { P. baldaccii } \\
\text { Females } \\
\text { PR_152 }\end{array}$} & \multirow{2}{*}{$\begin{array}{c}\text { P. enigmaticus } \\
\text { Females } \\
\text { PR_193 }\end{array}$} & \multirow{2}{*}{$\begin{array}{c}\boldsymbol{P} \text {. goodeyi } \\
\text { Females } \\
\text { PR_14 }\end{array}$} & \multicolumn{4}{|c|}{ P. israelensis } \\
\hline Sample code & & & & $\begin{array}{l}\text { Females } \\
\text { PR_82 }\end{array}$ & $\begin{array}{l}\text { Males } \\
\text { PR_82 }\end{array}$ & $\begin{array}{l}\text { Females } \\
\text { PR_79 }\end{array}$ & $\begin{array}{l}\text { Females } \\
\text { PR_11 }\end{array}$ \\
\hline $\begin{array}{c}\text { Locality } \\
\mathrm{n}\end{array}$ & $\begin{array}{c}\text { Cantillana, } \\
\text { Sevilla } \\
11\end{array}$ & $\begin{array}{c}\text { La Almunia, } \\
\text { Zaragoza } \\
5\end{array}$ & $\begin{array}{l}\text { Córdoba, } \\
\text { Córdoba } \\
8\end{array}$ & $\begin{array}{c}\text { Martos, Jaén } \\
7\end{array}$ & $\begin{array}{c}\text { Martos, Jaén } \\
4\end{array}$ & $\begin{array}{c}\text { Valenzuela, } \\
\text { Córdoba } \\
7\end{array}$ & $\begin{array}{l}\text { Córdoba, } \\
\text { Córdoba } \\
3\end{array}$ \\
\hline $\mathrm{L}$ & $\begin{array}{c}309.2 \pm 25.0 \\
(272-354)\end{array}$ & $\begin{array}{c}364.6 \pm 23.5 \\
(324-383)\end{array}$ & $\begin{array}{c}411.1 \pm 10.5 \\
(396-427)\end{array}$ & $\begin{array}{c}396.4 \pm 36.5 \\
(345-441)\end{array}$ & $\begin{array}{l}420.3 \pm 25 \\
(388-441)\end{array}$ & $\begin{array}{c}431.6 \pm 26.9 \\
(400-464)\end{array}$ & $\begin{array}{c}472.7 \pm 27.0 \\
(446-500)\end{array}$ \\
\hline$a^{*}$ & $\begin{array}{c}20.5 \pm 2.7 \\
(14.7-25.3)\end{array}$ & $\begin{array}{c}19.9 \pm 1.5 \\
(17.6-21.6)\end{array}$ & $\begin{array}{c}22.8 \pm 2.6 \\
(19.9-26.8)\end{array}$ & $\begin{array}{c}25.1 \pm 1.4 \\
(23.5-27.2)\end{array}$ & $\begin{array}{l}30.5 \pm 3.6 \\
(27.5-35.1)\end{array}$ & $\begin{array}{c}21.0 \pm 2.4 \\
(18.0-23.6)\end{array}$ & $\begin{array}{c}25.2 \pm 1.4 \\
(23.6-26.2)\end{array}$ \\
\hline $\mathrm{b}$ & $\begin{array}{l}3.8 \pm 0.4 \\
(3.4-4.4)\end{array}$ & $\begin{array}{l}3.8 \pm 0.2 \\
(3.6-4.2)\end{array}$ & $\begin{array}{l}3.9 \pm 0.2 \\
(3.5-4.2)\end{array}$ & $\begin{array}{l}3.9 \pm 0.3 \\
(3.5-4.3)\end{array}$ & $\begin{array}{c}4.9 \pm 0.24 \\
(4.5-5.1)\end{array}$ & $\begin{array}{l}4.1 \pm 0.4 \\
(3.8-4.8)\end{array}$ & $\begin{array}{l}4.2 \pm 0.3 \\
(3.9-4.5)\end{array}$ \\
\hline c & $\begin{array}{l}10.0 \pm 1.9 \\
(8.0-14.8)\end{array}$ & $\begin{array}{c}14.6 \pm 1.6 \\
(12.0-16.0)\end{array}$ & $\begin{array}{c}11.7 \pm 0.6 \\
(10.7-12.2)\end{array}$ & $\begin{array}{c}13.8 \pm 1.1 \\
(12.2-15.0)\end{array}$ & $\begin{array}{c}11.4 \pm 1.1 \\
(10.5-13.0)\end{array}$ & $\begin{array}{l}11.8 \pm 1.6 \\
(9.2-14.4)\end{array}$ & $\begin{array}{l}10.5 \pm 1.1 \\
(9.4-11.6)\end{array}$ \\
\hline$c^{\prime}$ & $\begin{array}{l}3.5 \pm 0.1 \\
(3.4-3.7)\end{array}$ & $\begin{array}{l}2.7 \pm 0.1 \\
(2.5-2.8)\end{array}$ & $\begin{array}{l}3.6 \pm 0.2 \\
(3.2-3.8)\end{array}$ & $\begin{array}{l}3.0 \pm 0.4 \\
(2.6-3.5)\end{array}$ & $\begin{array}{c}3.5 \pm 0.22 \\
(3.4-3.9)\end{array}$ & $\begin{array}{l}3.4 \pm 0.4 \\
(2.9-4.0)\end{array}$ & $\begin{array}{l}4.0 \pm 0.6 \\
(3.3-4.5)\end{array}$ \\
\hline $\mathrm{V}$ or $\mathrm{T}$ & $\begin{array}{c}79.8 \pm 1.0 \\
(77.5-81.2)\end{array}$ & $\begin{array}{l}83.4 \pm 1.3 \\
(81.2-84.4)\end{array}$ & $\begin{array}{c}81.7 \pm 0.5 \\
(81.0-82.4)\end{array}$ & $\begin{array}{c}81.5 \pm 1.1 \\
(79.9-83.4)\end{array}$ & $\begin{array}{c}47.5 \pm 4.6 \\
(44.8-52.8)\end{array}$ & $\begin{array}{c}80.3 \pm 1.0 \\
(78.7-81.8)\end{array}$ & $\begin{array}{c}78.0 \pm 2.0 \\
(76.1-80.0)\end{array}$ \\
\hline G1 & $\begin{array}{l}32.7 \pm 4.9 \\
(27.0-38.9)\end{array}$ & $\begin{array}{c}33.7 \pm 7.8 \\
(23.1-42.9)\end{array}$ & $\begin{array}{c}30.8 \pm 1.9 \\
(29.2-33.9)\end{array}$ & $\begin{array}{l}35.9 \pm 10.2 \\
(28.7-43.1)\end{array}$ & (12) & $\begin{array}{c}33.0 \pm 2.7 \\
(30.0-37.1)\end{array}$ & $\begin{array}{c}33.8 \pm 3.9 \\
(29.4-36.4)\end{array}$ \\
\hline Stylet length & $\begin{array}{l}30.5 \pm 1.1 \\
(29.0-33.0)\end{array}$ & $\begin{array}{l}27.6 \pm 0.9 \\
(27.0-29.0)\end{array}$ & $\begin{array}{l}49.6 \pm 2.6 \\
(46.0-53.0)\end{array}$ & $\begin{array}{l}26.7 \pm 1.5 \\
(24.0-29.0)\end{array}$ & $\begin{array}{c}14.5 \pm 0.6 \\
(14.0-15.0)\end{array}$ & $\begin{array}{l}26.0 \pm 1.2 \\
(25.0-28.0)\end{array}$ & $\begin{array}{l}26.5 \pm 0.5 \\
(26.0-27.0)\end{array}$ \\
\hline Conus length & $\begin{array}{l}21.2 \pm 1.5 \\
(19.0-24.0)\end{array}$ & $\begin{array}{c}17.5 \pm 0.9 \\
(17.0-19.0)\end{array}$ & $\begin{array}{l}40.2 \pm 2.6 \\
(37.0-44.0)\end{array}$ & $\begin{array}{c}16.5 \pm 1.6 \\
(14.5-19.0)\end{array}$ & $\begin{array}{l}8.3 \pm 0.4 \\
(8.0-8.8)\end{array}$ & $\begin{array}{c}16.6 \pm 0.7 \\
(15.5-17.5)\end{array}$ & $\begin{array}{c}16.5 \pm 0.5 \\
(16.0-17.0)\end{array}$ \\
\hline $\mathrm{m}$ & $\begin{array}{c}69.3 \pm 2.9 \\
(63.3-72.7)\end{array}$ & $\begin{array}{c}63.4 \pm 1.2 \\
(62.5-65.5)\end{array}$ & $\begin{array}{c}80.9 \pm 1.4 \\
(79.2-83.0)\end{array}$ & $\begin{array}{c}61.7 \pm 3.2 \\
(57.7-66.7)\end{array}$ & $\begin{array}{c}57.5 \pm 3.1 \\
(53.3-60.7)\end{array}$ & $\begin{array}{c}64.1 \pm 2.2 \\
(62.0-68.0)\end{array}$ & $\begin{array}{c}62.3 \pm 0.7 \\
(61.5-63.0)\end{array}$ \\
\hline DGO & $\begin{array}{l}5.5 \pm 0.7 \\
(4.5-6.5)\end{array}$ & $\begin{array}{l}5.9 \pm 1.6 \\
(4.0-7.5)\end{array}$ & $\begin{array}{l}5.3 \pm 0.4 \\
(4.5-5.5)\end{array}$ & $\begin{array}{l}6.4 \pm 0.5 \\
(5.5-7.0)\end{array}$ & $\begin{array}{l}5.3 \pm 0.6 \\
(5.0-6.0)\end{array}$ & $\begin{array}{l}6.6 \pm 0.7 \\
(6.0-7.5)\end{array}$ & $\begin{array}{l}5.8 \pm 0.3 \\
(5.5-6.0)\end{array}$ \\
\hline $\mathrm{O}$ & $\begin{array}{c}17.9 \pm 2.5 \\
(15.2-22.4)\end{array}$ & $\begin{array}{c}21.5 \pm 6.0 \\
(14.8-27.8)\end{array}$ & $\begin{array}{l}10.6 \pm 1.1 \\
(8.8-12.0)\end{array}$ & $\begin{array}{c}24.1 \pm 2.0 \\
(21.2-27.1)\end{array}$ & $\begin{array}{l}36.3 \pm 3.4 \\
(33.3-40.0)\end{array}$ & $\begin{array}{c}25.3 \pm 2.4 \\
(22.2-28.3)\end{array}$ & $\begin{array}{c}22.0 \pm 1.2 \\
(20.8-23.1)\end{array}$ \\
\hline Lip width & $\begin{array}{l}5.5 \pm 0.5 \\
(4.5-6.0)\end{array}$ & $\begin{array}{l}7.6 \pm 0.4 \\
(7.0-8.0)\end{array}$ & $\begin{array}{l}5.1 \pm 0.2 \\
(5.0-5.5)\end{array}$ & $\begin{array}{l}6.7 \pm 0.6 \\
(6.0-7.5)\end{array}$ & $\begin{array}{l}3.4 \pm 0.5 \\
(3.0-4.0)\end{array}$ & $\begin{array}{l}8.8 \pm 0.3 \\
(8.5-9.0)\end{array}$ & $\begin{array}{l}8.3 \pm 0.8 \\
(7.5-9.0)\end{array}$ \\
\hline $\begin{array}{l}\text { Median bulb } \\
\text { length }\end{array}$ & $\begin{array}{c}19.7 \pm 2.5 \\
(17.0-24.0)\end{array}$ & $\begin{array}{l}25.0 \pm 1.0 \\
(24.0-26.0)\end{array}$ & $\begin{array}{c}20.6 \pm 2.1 \\
(17.0-23.0)\end{array}$ & $\begin{array}{c}20.4 \pm 2.8 \\
(16.5-24.0)\end{array}$ & $\begin{array}{c}16.7 \pm 0.6 \\
(16.0-17.0)\end{array}$ & $\begin{array}{c}25.8 \pm 3.1 \\
(23.0-31.0)\end{array}$ & $\begin{array}{c}24.3 \pm 2.1 \\
(22.0-26.0)\end{array}$ \\
\hline Median bulb width & $\begin{array}{l}8.6 \pm 0.7 \\
(7.5-9.5)\end{array}$ & $\begin{array}{c}11.2 \pm 0.8 \\
(10.5-12.0)\end{array}$ & $\begin{array}{c}10.9 \pm 0.3 \\
(10.5-11.5)\end{array}$ & $\begin{array}{l}9.8 \pm 0.6 \\
(9.0-10.5)\end{array}$ & $\begin{array}{l}7.8 \pm 0.3 \\
(7.5-8.0)\end{array}$ & $\begin{array}{c}11.9 \pm 1.2 \\
(10.5-14.0)\end{array}$ & $\begin{array}{l}8.5 \pm 0.5 \\
(8.0-9.0)\end{array}$ \\
\hline $\begin{array}{l}\text { Anterior end to } \\
\text { center median bulb }\end{array}$ & $\begin{array}{c}47.6 \pm 3.1 \\
(43.0-52.0)\end{array}$ & $\begin{array}{l}53.6 \pm 3.0 \\
(50.0-57.0)\end{array}$ & $\begin{array}{l}73.7 \pm 2.8 \\
(70.0-77.0)\end{array}$ & $\begin{array}{l}57.1 \pm 3.0 \\
(52.5-60.5)\end{array}$ & $\begin{array}{c}52.6 \pm 5.1 \\
(45.0-56.0)\end{array}$ & $\begin{array}{c}60.2 \pm 3.8 \\
(55.0-65.0)\end{array}$ & $\begin{array}{c}60.7 \pm 1.5 \\
(59.0-62.0)\end{array}$ \\
\hline $\mathrm{MB}$ & $\begin{array}{c}59.0 \pm 1.2 \\
(57.6-60.5)\end{array}$ & $\begin{array}{c}55.8 \pm 1.0 \\
(54.4-56.8)\end{array}$ & $\begin{array}{c}68.5 \pm 3.4 \\
(62.6-72.8)\end{array}$ & $\begin{array}{c}56.4 \pm 0.9 \\
(55.2-57.6)\end{array}$ & (1) & $\begin{array}{c}56.8 \pm 1.8 \\
(54.7-60.0)\end{array}$ & $\begin{array}{c}54.2 \pm 0.9 \\
(53.2-54.9)\end{array}$ \\
\hline $\begin{array}{l}\text { Nerve ring to } \\
\text { anterior end }\end{array}$ & $\begin{array}{c}62.1 \pm 4.0 \\
(57.0-68.0)\end{array}$ & $\begin{array}{l}70.4 \pm 4.0 \\
(66.0-75.0)\end{array}$ & $\begin{array}{r}87.5 \pm 7.4 \\
(78.0-96.0)\end{array}$ & $\begin{array}{l}75.5 \pm 5.3 \\
(68.0-85.5)\end{array}$ & $\begin{array}{c}62.3 \pm 5.3 \\
(58.5-66.0)\end{array}$ & $\begin{array}{l}78.4 \pm 5.9 \\
(71.0-89.5)\end{array}$ & $\begin{array}{l}78.3 \pm 3.2 \\
(76.0-82.0)\end{array}$ \\
\hline $\begin{array}{l}\text { Excretory pore to } \\
\text { anterior end }\end{array}$ & $\begin{array}{l}75.3 \pm 6.5 \\
(69.0-90.0)\end{array}$ & $\begin{array}{l}87.6 \pm 5.7 \\
(81.0-94.0)\end{array}$ & $\begin{array}{c}91.6 \pm 6.8 \\
(82.0-102.0)\end{array}$ & $\begin{array}{r}87.2 \pm 6.5 \\
(79.5-96.5)\end{array}$ & $\begin{array}{c}79.5 \pm 6.1 \\
(72.5-84.0)\end{array}$ & $\begin{array}{c}90.2 \pm 5.4 \\
(84.0-97.0)\end{array}$ & $\begin{array}{r}92.0 \pm 1.0 \\
(91.0-93.0)\end{array}$ \\
\hline Pharynx length & $\begin{array}{l}82.3 \pm 4.5 \\
(75.0-87.0)\end{array}$ & $\begin{array}{c}96.2 \pm 6.5 \\
(88.0-103.0)\end{array}$ & $\begin{array}{c}107 \pm 8.3 \\
(97.0-123.0)\end{array}$ & $\begin{array}{c}100.7 \pm 4.6 \\
(93.0-107.0)\end{array}$ & $\begin{array}{l}86.3 \pm 5.9 \\
(78.0-91.0)\end{array}$ & $\begin{array}{c}106.1 \pm 8.3 \\
(95.0-117.0)\end{array}$ & $\begin{array}{c}112.0 \pm 1.0 \\
(111-113)\end{array}$ \\
\hline $\begin{array}{l}\text { Maximum body } \\
\text { diam. }\end{array}$ & $\begin{array}{c}15.3 \pm 1.6 \\
(12.5-18.5)\end{array}$ & $\begin{array}{c}18.4 \pm 1.7 \\
(16.5-21.0)\end{array}$ & $\begin{array}{c}18.3 \pm 2.2 \\
(15.0-20.5)\end{array}$ & $\begin{array}{c}15.8 \pm 0.8 \\
(14.5-16.5)\end{array}$ & $\begin{array}{l}13.9 \pm 1.0 \\
(12.5-15.0)\end{array}$ & $\begin{array}{c}20.7 \pm 2.4 \\
(18.0-23.5)\end{array}$ & $\begin{array}{c}18.8 \pm 1.6 \\
(17.0-20.0)\end{array}$ \\
\hline Tail length & $\begin{array}{c}31.7 \pm 5.2 \\
(24.0-40.0)\end{array}$ & $\begin{array}{c}25.0 \pm 1.4 \\
(23.5-27.0)\end{array}$ & $\begin{array}{l}35.3 \pm 1.5 \\
(33.5-37.5)\end{array}$ & $\begin{array}{l}228.9 \pm 3.9 \\
(23.0-33.5)\end{array}$ & $\begin{array}{c}37.1 \pm 2.7 \\
(34.0-40.5)\end{array}$ & $\begin{array}{c}36.9 \pm 5.0 \\
(32.0-46.0)\end{array}$ & $\begin{array}{c}45.3 \pm 4.0 \\
(43.0-50.0)\end{array}$ \\
\hline Anal body diam. & $\begin{array}{l}9.0 \pm 1.2 \\
(7.0-11.0)\end{array}$ & $\begin{array}{l}9.4 \pm 0.4 \\
(9.0-10.0)\end{array}$ & $\begin{array}{l}9.9 \pm 0.6 \\
(9.0-10.5)\end{array}$ & $\begin{array}{l}9.8 \pm 0.5 \\
(9.0-10.5)\end{array}$ & $\begin{array}{c}10.5 \pm 0.4 \\
(44.8-52.8)\end{array}$ & $\begin{array}{c}11.0 \pm 1.1 \\
(10.0-12.5)\end{array}$ & $\begin{array}{c}11.5 \pm 1.3 \\
(10.5-13.0)\end{array}$ \\
\hline Spicules & (1) & (1) & (1) & (1) & $\begin{array}{l}21.8 \pm 0.96 \\
(21.0-23.0)\end{array}$ & (1) & (120) \\
\hline Gubernaculum & - & - & - & - & $\begin{array}{c}5.1 \pm 0.25 \\
(5.0-5.5)\end{array}$ & - & - \\
\hline
\end{tabular}

* Abbreviations: $\mathrm{a}=$ body length/greatest body diameter; $\mathrm{b}=$ body length/distance from anterior end to pharyngo-intestinal junction; $\mathrm{DGO}=$ distance between stylet base and orifice of dorsal pharyngeal gland; $\mathrm{c}=$ body length/tail length; $\mathrm{c}^{\prime}=$ tail length/tail diameter at anus or cloaca; G1 = anterior genital branch length expressed as percentage $(\%)$ of the body length; $\mathrm{L}=$ overall body length; $\mathrm{m}=$ length of conus as percentage of total stylet length; $\mathrm{MB}=$ distance between anterior end of body and center of median pharyngeal bulb expressed as percentage $(\%)$ of the pharynx length; $\mathrm{n}=$ number of specimens on which measurements are based; $\mathrm{O}=\mathrm{DGO}$ as percentage of stylet length; $\mathrm{T}=$ distance from cloacal aperture to anterior end of testis expressed as percentage $(\%)$ of the body length; $\mathrm{V}=$ distance from body anterior end to vulva expressed as percentage (\%) of the body length. 
Molecular characterization: Molecular markers agree with the identification of this species. One D2-D3 of 28S rRNA (MW798292), one ITS (MW798337) and one COI gene sequences (MW797013) were generated for this species, and all of them were found to be identical to several accessions from P. enigmaticus deposited in GenBank such as, MN535546 for D2-D3 of 28S, MW319816 and MN535551 for the ITS region, and MW421686 for the COI gene sequences.

Paratylenchus goodeyi (Oostenbrink, 1953) Raski, 1962.

This species has been detected in several countries as the Netherlands [68,69], Belgium, Germany, and England [24,69,70], Kazakhstan [71], Moldavia [72], Karelia (Russia) [73], Spain [48], Poland [33,74,75], and Slovakia [76]. However, only measurements are presented in Oostenbrink [68], Szczygiel [74], Castillo et al. [48], Brzeski [33,75] and Singh et al. [24].

The Spanish population from Córdoba characterized in this study coincides mainly with the original description of the species (Table 6, Figure 13) at exception of the position of the excretory pore $(82.0-102.0 \mu \mathrm{m}$ vs. $64 \mu \mathrm{m})$. This species matches well with other populations, as the Spanish population described by Castillo et al. [48], at exception of a shorter body [396-427 $\mu \mathrm{m}$ vs. $410-450 \mu \mathrm{m}$ ] and smaller $\mathrm{c}^{\prime}$ ratio (3.2-3.8 vs. $\left.4.1-4.7\right)$.

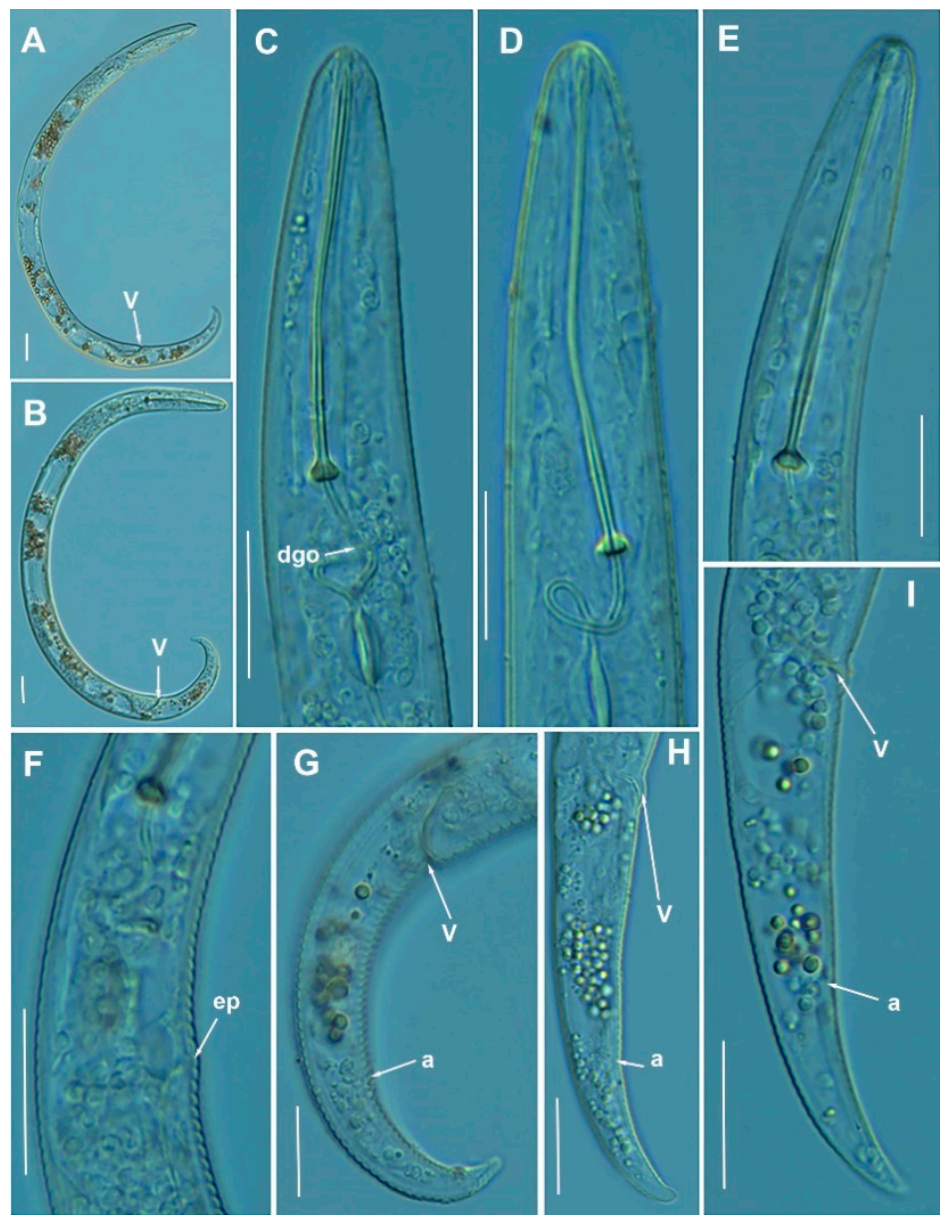

Figure 13. Light photomicrographs of Paratylenchus goodeyi (Oostenbrink, 1953) Raski, 1962. (A,B): Entire female with vulva arrowed; $(\mathbf{C}-\mathbf{E})$ : Female lip region; $(\mathbf{F})$ : Detail of excretory pore; (G-I): Female posterior region with vulva and anus (arrowed). Scale bars $(\mathbf{A}-\mathbf{I}=20 \mu \mathrm{m})$. (Abbreviations: a $=$ anus; $\mathrm{dgo}=$ pharyngeal dorsal gland orifice; $\mathrm{ep}=$ excretory pore; $\mathrm{V}=$ vulva).

Molecular characterization: Molecular markers of this population agree with those provided for this species by Singh et al. [24]. Two D2-D3 of 28S rRNA (MW798293MW798294), two ITS (MW798338- MW798339) and two COI gene sequences (MW797014- 
MW797015) were generated in this study without intraspecific sequence variations for this population. The D2-D3 of $28 \mathrm{~S}$ rRNA sequences were $99 \%$ similar (differing by 9 nucleotides) with P. goodeyi from Belgium (MW413631-MW413633). The ITS sequences were $96 \%$ similar (differing by 27 nucleotides and 11 indels) with the P. goodeyi sequence MW423594 and finally, the COI gene sequences showed $95 \%$ similarity (differing by 19 nucleotides) with the accessions from P. goodeyi deposited in GenBank (MW421648-MW421649).

Paratylenchus hamatus Thorne \& Allen, 1950 and Paratylenchus baldaccii Raski, 1975.

These species are closely related morphologically to other species such as P. tenuicaudatus. Van den Berg et al. [23] included P. tenuicaudatus within the P. hamatus sensu stricto, $P$. baldaccii and two other putative species within the P. hamatus "species complex". Only one character has been pointed by different authors to separate P. hamatus from P. baldaccii, viz. slenderer and sharply conoid female tail tip and male tail tips in P. hamatus vs. P. baldaccii which was described as finely rounded to almost acute [75,77]. Paratylenchus baldaccii is described morphologically in this study (Table 6, Figure 14) with molecular markers provided too (see description below) and different to other molecular species descriptions within the P. hamatus "species complex" [23]. Our population of $P$. baldaccii matches with the original description of the species, but in our samples it is really very difficult to separate $P$. baldaccii and P. hamatus only based on morphological traits. However, topotypes of $P$. baldaccii should be necessary in order to assign a definitive molecular marker association between morphology and molecular differences in this complex species group. Specimens of $P$. hamatus from fig orchards at the type locality (Planada, CA, USA) were molecularly characterized by Van den Berg et al. [23]. Paratylenchus hamatus has not been described before in Spain and constitute a first record for the country.

Paratylenchus hamatus has a worldwide distribution and has been reported in many countries including Australia, Belgium, Canada, Pakistan, etc. [36]. This species can cause damage in different crops as figs and several vegetables [36]. Different populations of this species have been found in our study in the rhizosphere of peach/rootstock peach $\times$ almond [GxN] (sample codes PR_44 and PR_207) and almond/rootstock almond orchards (sample codes PR_115 and PR_187). Nematode soil population levels were high or very high (42400, 4212, 2042 and 8250 individuals per $500 \mathrm{~cm}^{3}$ of soil) in peach orchards sample codes PR_44, PR_115, PR_187 and PR_207, respectively. However, peach trees did not show any apparent growth reduction or symptomatology associated with these high levels of nematodes in the soil. Morphologically, the Spanish populations studied herein (Figure 15, Table 7) were in the range of the described populations and topotypes from P. hamatus [23].

Paratylenchus baldaccii has been described in grapevine in Sicily (Italy), Bari (Italy) and South of France [77] and later in Italy [75] and Spain [44]. In our sampling has been recorded in one locality in a peach orchard at a density of 200 individuals per $500 \mathrm{cc}$ of soil.

Molecular characterization: Five D2-D3 of 28S rRNA (MW798295- MW798299), two ITS (MW798340-MW798341), and two COI gene sequences (MW797016-MW797017) of $P$. hamatus were generated in this study without intraspecific sequence variations. All of sequences were found to be, respectively, identical to KF242208, KF242248 and MN711355, accessions belonging to $P$. hamatus from USA $[23,78]$.

For P. baldaccii, two D2-D3 of 28S rRNA (MW798290-MW798291), two ITS (MW798335MW798336), and one COI gene sequences (MW797012) were generated herein without intraspecific sequence variations. The closest Paratylenchus sequences to $P$. baldaccii were those of P. pedrami sp. nov. with 96, 93 and 90\% similarity (differing by 30, 45 and 26 nucleotides) for the D2-D3 of 28S rRNA (MW798283-MW798285), ITS region (MW798329MW798330) and COI gene (MW797009), respectively.

Paratylenchus holdemani Raski, 1975. 

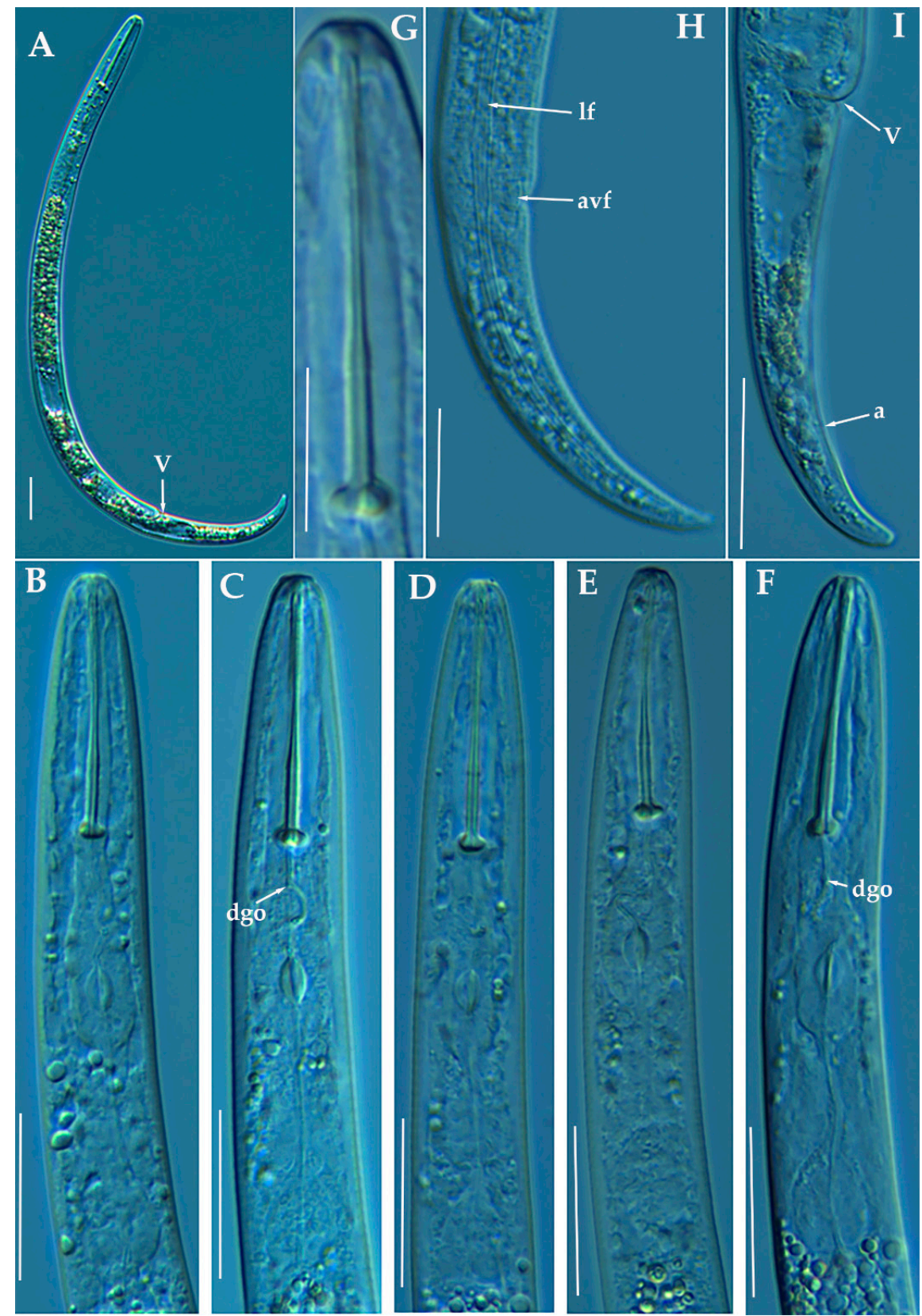

Figure 14. Light photomicrographs of Paratylenchus baldaccii Raski, 1975. (A): Entire female with vulva arrowed; (B-F): Female pharyngeal region; (G): Detail of female stylet; (H): Female posterior region showing lateral field and advulval flap (arrowed); (I): Female posterior region showing vulva and anus (arrowed). Scale bars (A-F, H,I $=20 \mu \mathrm{m} ; \mathbf{G}=10 \mu \mathrm{m})$. (Abbreviations: a = anus; avf $=$ advulval flap; dgo = pharyngeal dorsal gland orifice; $\mathrm{lf}=$ lateral field; $\mathrm{V}=$ vulva).

This species has been described from Santa Ana, El Salvador [77] and also reported in Czech Republic [33] and Belgium [24]. It resembles morphologically P. hamatus and P. baldaccii but differs from them in having a shorter female stylet [36]. The morphology and morphometry of the Spanish population from Martos, Jaén province (Southern Spain) (Table 6, Figure 16) agrees with the original species description and other populations described, as well as molecularly in various molecular markers to populations sequenced for this species [24]. Minor differences were found in longer female body (345-441 $\mu \mathrm{m}$ vs. $290-350 \mu \mathrm{m})$, higher a ratio (23.5-27.2 vs. 19-24) and longer stylet (24-29 $\mu \mathrm{m}$ vs. $21-23 \mu \mathrm{m})$ in comparison to paratypes. However, later species descriptions increased the overlapping ranges for some important characters as longer body $(285-475 \mu \mathrm{m})$, longer stylet 
(19.0-26.1 $\mu \mathrm{m})$ [24]. In our case the morphometrics and molecular data were coincident with Singh et al. [24] and increase the morphological traits range of the species even further than the original description of the species. The presence of males is also reported in the Spanish population. The integrative taxonomical identification of this population confirms the morphometrical plasticity of this species. Only one population has been found in an almond orchard in Martos, Jaén province with 4735 individuals per $500 \mathrm{~cm}^{3}$ of soil. This species has not been described before in Spain and constitutes a first report for the country and expand their distribution in Europe.
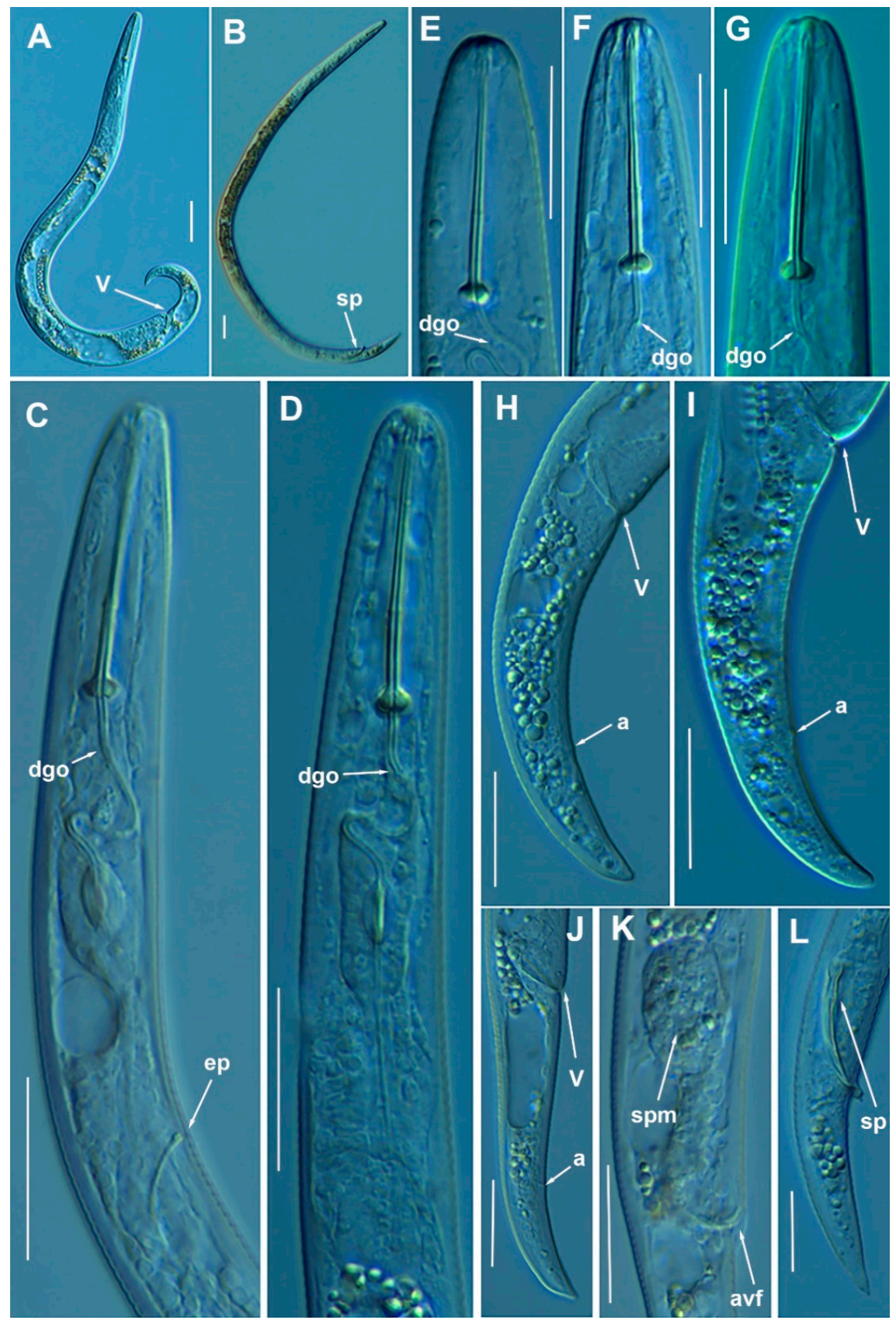

Figure 15. Light photomicrographs of Paratylenchus hamatus Thorne \& Allen, 1950. (A): Entire female with vulva arrowed; (B): Entire male with spicules arrowed; (C,D): Female pharyngeal region; (E-G): Female lip region; $(\mathbf{H}-\mathbf{J})$ : Female posterior region with vulva and anus (arrowed); (K): Detail of vulva showing spermatheca and advulval flap (arrowed); (L): Male tail with spicules arrowed. Scale bars $(\mathbf{A}-\mathbf{L}=20 \mu \mathrm{m})$. (Abbreviations: $\mathrm{a}=$ anus; avf = advulval flap; dgo = pharyngeal dorsal gland orifice; $\mathrm{ep}=$ excretory pore; $\mathrm{spm}=$ spermatheca; $\mathrm{sp}=$ spicules; $\mathrm{V}=$ vulva). 
Table 7. Morphometrics of Paratylenchus hamatus Thorne \& Allen, 1950 from several localities in Spain. All measurements are in $\mu \mathrm{m}$ and in the form: mean \pm s.d. (range).

\begin{tabular}{|c|c|c|c|c|c|}
\hline Measurements and Ratios & Females & Females & Females & Females & Males \\
\hline Sample code & PR_44 & PR_115 & PR_207 & PR_187 & PR_187 \\
\hline $\begin{array}{l}\text { Locality } \\
\mathrm{n}\end{array}$ & $\begin{array}{c}\text { Gibraleón, Huelva } \\
4\end{array}$ & $\begin{array}{c}\text { Lúcar, Almería } \\
4\end{array}$ & $\begin{array}{c}\text { Sástago, Zaragoza } \\
5\end{array}$ & $\begin{array}{c}\text { Ariza, Zaragoza } \\
15\end{array}$ & $\begin{array}{c}\text { Ariza, Zaragoza } \\
2 \\
\end{array}$ \\
\hline $\mathrm{L}$ & $\begin{array}{c}362 \pm 11.0 \\
(347-373)\end{array}$ & $\begin{array}{c}386.5 \pm 28.8 \\
(355-420)\end{array}$ & $\begin{array}{c}421.6 \pm 46.9 \\
(377-487)\end{array}$ & $\begin{array}{c}373.5 \pm 31.4 \\
(327-433.5)\end{array}$ & 744,789 \\
\hline$a^{*}$ & $\begin{array}{l}22.2 \pm 1.4 \\
(20.4-23.4)\end{array}$ & $\begin{array}{l}20.7 \pm 1.8 \\
(19.2-23.3)\end{array}$ & $\begin{array}{l}20.5 \pm 3.7 \\
(16.5-24.9)\end{array}$ & $\begin{array}{l}20.2 \pm 3.1 \\
(13.5-25.3)\end{array}$ & $40.5,45.1$ \\
\hline $\mathrm{b}$ & $\begin{array}{l}4.0 \pm 0.2 \\
(3.9-4.2)\end{array}$ & $\begin{array}{l}3.8 \pm 0.3 \\
(3.4-4.1)\end{array}$ & $\begin{array}{l}4.6 \pm 0.4 \\
(4.3-5.2)\end{array}$ & $\begin{array}{l}3.8 \pm 0.2 \\
(3.5-4.4)\end{array}$ & $4.7,6.7$ \\
\hline c & $\begin{array}{l}12.9 \pm 0.8 \\
(11.7-13.6)\end{array}$ & $\begin{array}{l}13.9 \pm 1.7 \\
(11.7-15.4)\end{array}$ & $\begin{array}{l}13.6 \pm 1.8 \\
(11.5-16.2)\end{array}$ & $\begin{array}{c}12.6 \pm 1.0 \\
(10.4 \pm 14.6)\end{array}$ & $17.5,18.6$ \\
\hline$c^{\prime}$ & $\begin{array}{l}3.3 \pm 0.1 \\
(3.2-3.4)\end{array}$ & $\begin{array}{l}3.0 \pm 0.3 \\
(2.6-3.3)\end{array}$ & $\begin{array}{l}3.1 \pm 0.3 \\
(2.9-3.6)\end{array}$ & $\begin{array}{l}2.9 \pm 0.3 \\
(2.3-3.6)\end{array}$ & $3.5,4.3$ \\
\hline $\mathrm{V}$ or $\mathrm{T}$ & $\begin{array}{c}81.7 \pm 0.9 \\
(80.4 \pm 82.6)\end{array}$ & $\begin{array}{l}81.9 \pm 0.4 \\
(81.5-82.2)\end{array}$ & $\begin{array}{l}81.8 \pm 1.3 \\
(80.4-83.6)\end{array}$ & $\begin{array}{l}82.0 \pm 1.1 \\
(80.1-84.2)\end{array}$ & $53.0,59.1$ \\
\hline G1 & $\begin{array}{r}41.7 \pm 6.9 \\
(33.3-47.7)\end{array}$ & $\begin{array}{l}39.7 \pm 4.9 \\
(35.6-45.6)\end{array}$ & $\begin{array}{l}55.9 \pm 3.2 \\
(52.3-59.9)\end{array}$ & $\begin{array}{l}46.5 \pm 7.6 \\
(33.0-53.5)\end{array}$ & - \\
\hline Stylet length & $\begin{array}{l}31.1 \pm 1.9 \\
(29.0-33.0)\end{array}$ & $\begin{array}{l}33.4 \pm 2.1 \\
(31.5-36.0)\end{array}$ & $\begin{array}{l}30.1 \pm 0.7 \\
(29.0-31.0)\end{array}$ & $\begin{array}{l}30.9 \pm 1.1 \\
(28.0-32.5)\end{array}$ & $16.5,19.5$ \\
\hline Conus length & $\begin{array}{l}20.8 \pm 1.8 \\
(19.0-23.0)\end{array}$ & $\begin{array}{l}22.5 \pm 1.7 \\
(21.0-25.0)\end{array}$ & $\begin{array}{c}20.2 \pm 2.2 \\
(18.0-23.0)\end{array}$ & $\begin{array}{l}19.8 \pm 1.1 \\
(18.0-21.5)\end{array}$ & $8.0,8.0$ \\
\hline $\mathrm{m}$ & $\begin{array}{l}66.6 \pm 2.8 \\
(65.0-70.8)\end{array}$ & $\begin{array}{l}67.4 \pm 2.6 \\
(64.7-69.8)\end{array}$ & $\begin{array}{l}67.0 \pm 5.7 \\
(62.1-74.2)\end{array}$ & $\begin{array}{l}59.8 \pm 2.8 \\
(60.0-69.4)\end{array}$ & $41.0,48.5$ \\
\hline DGO & $\begin{array}{l}3.8 \pm 0.3 \\
(3.5-4.0)\end{array}$ & $\begin{array}{l}7.1 \pm 0.9 \\
(6.0-8.0)\end{array}$ & $\begin{array}{l}6.5 \pm 0.8 \\
(5.5-7.5)\end{array}$ & - & $4.5,5.5$ \\
\hline $\mathrm{O}$ & $\begin{array}{l}12.0 \pm 0.3 \\
(11.7-12.3)\end{array}$ & $\begin{array}{l}21.4 \pm 3.0 \\
(18.8-25.4)\end{array}$ & $\begin{array}{l}21.6 \pm 2.4 \\
(18.3-24.2)\end{array}$ & - & $27.3,28.2$ \\
\hline Lip width & $\begin{array}{l}4.5 \pm 0.4 \\
(4.0-5.0)\end{array}$ & $\begin{array}{l}6.3 \pm 0.3 \\
(6.0-6.5)\end{array}$ & $\begin{array}{l}6.4 \pm 0.4 \\
(6.0-7.0)\end{array}$ & $\begin{array}{l}6.2 \pm 0.4 \\
(5.5-7.0)\end{array}$ & $6.5,7.5$ \\
\hline Median bulb length & - & $\begin{array}{l}22.8 \pm 2.9 \\
(21.0-27.0)\end{array}$ & $\begin{array}{l}26.8 \pm 1.0 \\
(26.0-28.0)\end{array}$ & $\begin{array}{l}17.9 \pm 2.0 \\
(13.5-20.0)\end{array}$ & $10.5,12.5$ \\
\hline Median bulb width & - & $\begin{array}{l}9.4 \pm 1.9 \\
(7.0-11.5)\end{array}$ & $\begin{array}{l}11.0 \pm 0.8 \\
(10.0-12.0)\end{array}$ & $\begin{array}{l}9.4 \pm 1.4 \\
(7.0-12.0)\end{array}$ & $8.0,10.0$ \\
\hline $\begin{array}{l}\text { Anterior end to center } \\
\text { median bulb }\end{array}$ & $\begin{array}{l}52.5 \pm 1.7 \\
(50.0-54.0)\end{array}$ & $\begin{array}{l}56.0 \pm 1.8 \\
(54.0-58.0)\end{array}$ & $\begin{array}{l}55.0 \pm 2.0 \\
(53.0-58.0)\end{array}$ & $\begin{array}{l}56.1 \pm 3.7 \\
(51.0-64.0)\end{array}$ & $64.5,70.0$ \\
\hline MB & $\begin{array}{l}58.0 \pm 2.2 \\
(55.6-60.2)\end{array}$ & $\begin{array}{l}55.4 \pm 2.1 \\
(52.9-58.1)\end{array}$ & $\begin{array}{l}60.5 \pm 3.6 \\
(56.8-65.1)\end{array}$ & $\begin{array}{l}57.3 \pm 2.6 \\
(53.2-62.2)\end{array}$ & $44.6,55.1$ \\
\hline Nerve ring to anterior end & $\begin{array}{l}71.3 \pm 5.4 \\
(67.0-79.0)\end{array}$ & $\begin{array}{l}74.3 \pm 6.9 \\
(67.0-83.0)\end{array}$ & $\begin{array}{l}66.0 \pm 1.7 \\
(63.0-67.0)\end{array}$ & 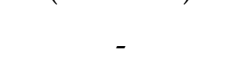 & $85.5,87.0$ \\
\hline $\begin{array}{l}\text { Excretory pore to } \\
\text { anterior end }\end{array}$ & $\begin{array}{l}82.0 \pm 5.2 \\
(76.0-88.0)\end{array}$ & $\begin{array}{l}85.9 \pm 7.1 \\
(78.5-92.0)\end{array}$ & $\begin{array}{l}83.8 \pm 2.3 \\
(81.0-87.0)\end{array}$ & $\begin{array}{c}87.8 \pm 6.7 \\
(77.5-100.0)\end{array}$ & $104.0,108.0$ \\
\hline Pharynx length & $\begin{array}{l}90.5 \pm 3.1 \\
(88.0-95.0)\end{array}$ & $\begin{array}{l}101.3 \pm 5.6 \\
(93.0-105.0)\end{array}$ & $\begin{array}{l}91.0 \pm 3.8 \\
(86.0-95.0)\end{array}$ & $\begin{array}{c}98.3 \pm 6.3 \\
(85.5-109.0)\end{array}$ & $117.0,157.0$ \\
\hline Maximum body diam. & $\begin{array}{l}16.4 \pm 0.8 \\
(15.5-17.0)\end{array}$ & $\begin{array}{c}18.8 \pm 2.3 \\
(16.0-21.5)\end{array}$ & $\begin{array}{l}21.2 \pm 4.9 \\
(18.0-29.0)\end{array}$ & $\begin{array}{c}19.0 \pm 3.7 \\
(17.0-27.5)\end{array}$ & $16.5,19.5$ \\
\hline Tail length & $\begin{array}{l}28.3 \pm 2.2 \\
(26.0-31.0)\end{array}$ & $\begin{array}{l}28.4 \pm 5.5 \\
(23.0-36.0)\end{array}$ & $\begin{array}{l}31.2 \pm 3.7 \\
(26.0-36.0)\end{array}$ & $\begin{array}{l}29.7 \pm 2.0 \\
(25.5-33.0)\end{array}$ & $40.0,45.0$ \\
\hline Anal body diam. & $\begin{array}{l}8.6 \pm 0.5 \\
(8.0-9.0)\end{array}$ & $\begin{array}{c}9.5 \pm 1.1 \\
(8.5-11.0)\end{array}$ & $\begin{array}{l}10.0 \pm 0.6 \\
(9.0-10.5)\end{array}$ & $\begin{array}{l}10.2 \pm 1.2 \\
(8.0-12.5)\end{array}$ & $10.5,11.5$ \\
\hline Spicules & - & - & - & - & $22.0,23.5$ \\
\hline Gubernaculum & - & - & - & - & $11.0,13.5$ \\
\hline
\end{tabular}

* Abbreviations: $\mathrm{a}=$ body length/greatest body diameter; $\mathrm{b}=$ body length/distance from anterior end to pharyngo-intestinal junction; $\mathrm{DGO}=$ distance between stylet base and orifice of dorsal pharyngeal gland; $\mathrm{c}=$ body length $/$ tail length; $\mathrm{c}^{\prime}=$ tail length/tail diameter at anus or cloaca; G1 = anterior genital branch length expressed as percentage (\%) of the body length; $\mathrm{L}=$ overall body length; $\mathrm{m}=$ length of conus as percentage of total stylet length; $\mathrm{MB}=$ distance between anterior end of body and center of median pharyngeal bulb expressed as percentage (\%) of the pharynx length; $\mathrm{n}=$ number of specimens on which measurements are based; $\mathrm{O}=\mathrm{DGO}$ as percentage of stylet length; $\mathrm{T}=$ distance from cloacal aperture to anterior end of testis expressed as percentage $(\%)$ of the body length; $\mathrm{V}=$ distance from body anterior end to vulva expressed as percentage (\%) of the body length. 

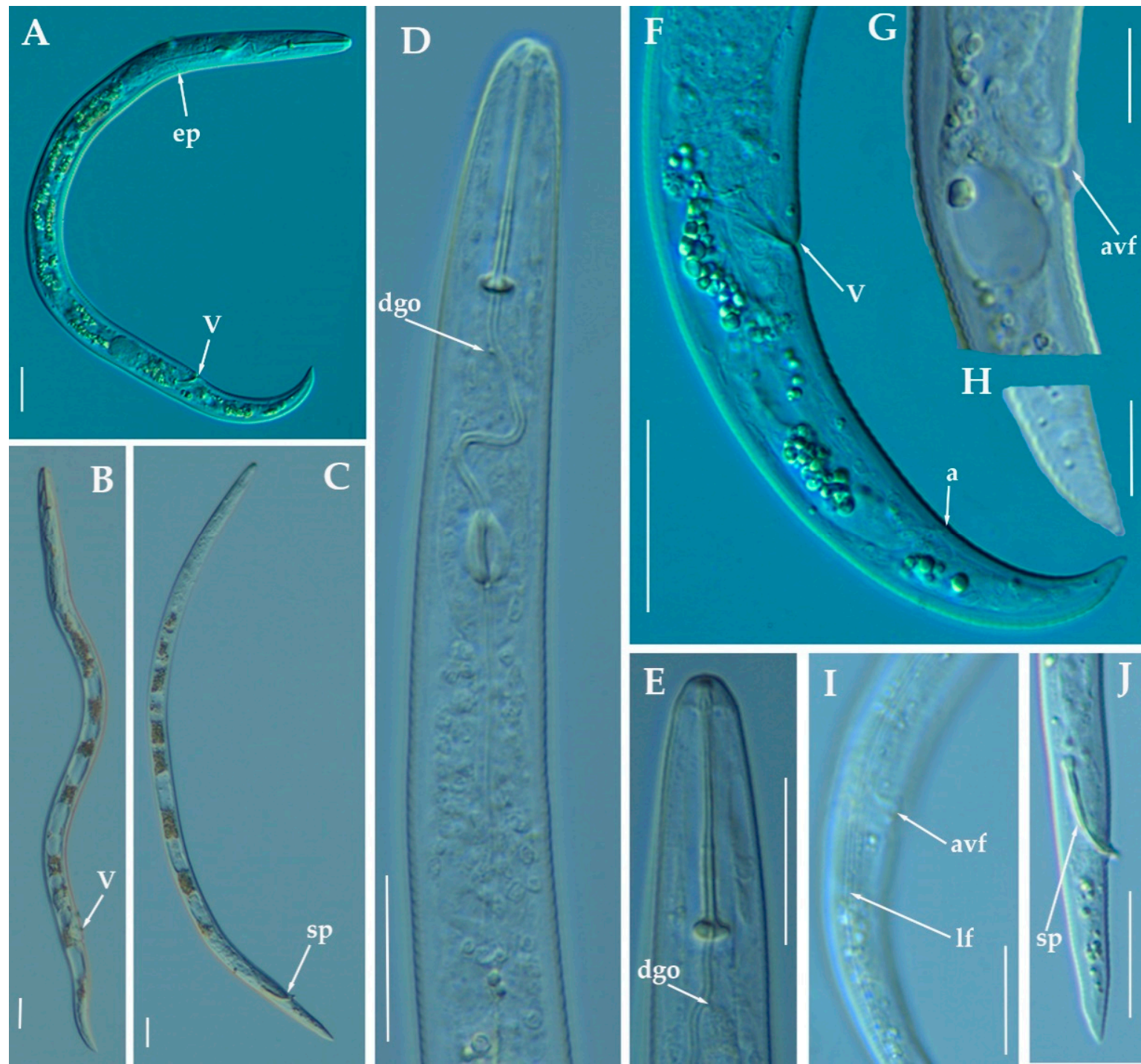

Figure 16. Light photomicrographs of Paratylenchus holdemani Raski, 1975. (A,B): Entire female with vulva arrowed; (C): Entire male with spicules arrowed; (D): Female pharyngeal region; E: Female lip region; F: Female posterior region with vulva and anus (arrowed); (G): Detail of vulva showing advulval flap (arrowed); (H): Detail of female tail tip; (I): Female posterior region showing lateral field and advulval flap (arrowed); (J): Male tail with spicules arrowed. Scale bars $(\mathbf{A}-\mathbf{F}, \mathbf{I}, \mathbf{J}=20 \mu \mathrm{m} ; \mathbf{G}-\mathbf{H}=10 \mu \mathrm{m})$. (Abbreviations: a= anus; avf= advulval flap; dgo= pharyngeal dorsal gland orifice; ep = excretory pore; $\mathrm{lf}=$ lateral field; $\mathrm{sp}=$ spicules; $\mathrm{V}=$ vulva).

Molecular characterization: One sequence from all regions were generated in this study, MW798300, MW798342 and MW797018 (D2-D3 of 28S rRNA, ITS and COI gene, respectively) being all of them identical to several accessions from P. holdemani deposited in GenBank, such as MW413642 for the D2-D3 of 28S, MW413596 for the ITS region and MW421652 for the COI gene [24].

Paratylenchus israelensis (Raski, 1973) Siddiqi, 1986.

This species was described in Shiller, Israel [79]. It is characterized by a strong sclerotization of the lip region. This species is similar to $P$. sheri, from which differs in its longer more robust stylet, stronger sclerotization of the lip region and different outline of lateral field in cross section [36]. The Spanish populations from two localities in Córdoba province (Southern Spain) fit the original description of P. israelensis and no differences were found (Table 6, Figure 17). Soil populations from almond orchards in Córdoba (sample code PR_011) and in Valenzuela (sample code PR_079) showed 368 and 320 individuals $/ 500 \mathrm{~cm}^{3}$ 
of soil, respectively. No symptoms of decline were apparently detected in the trees. This species has not been described before in Spain and constitute a first record for the country.
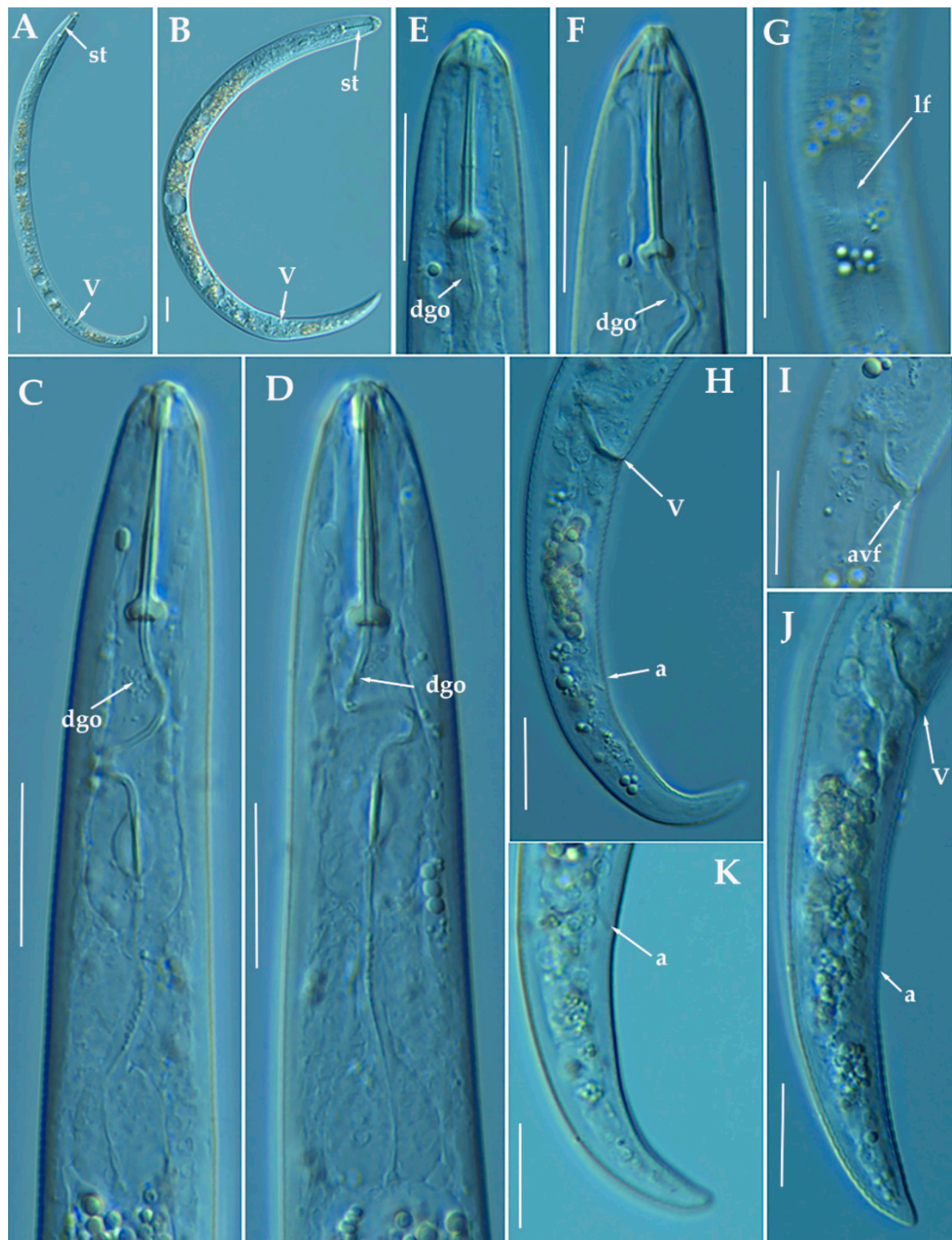

Figure 17. Light photomicrographs of Paratylenchus israelensis (Raski, 1973) Siddiqi, 1986. (A,B): Entire female with vulva arrowed; (C,D): Female pharyngeal region; (E,F): Female lip region; (G): Detail of lateral field at mid-body; (H,J): Female posterior region with vulva and anus (arrowed); (I): Detail of vulva showing advulval flap (arrowed); (K): Detail of female tail tip with anus arrowed. Scale bars $(\mathbf{A}-\mathbf{K}=20 \mu \mathrm{m})$. (Abbreviations: $\mathrm{a}=$ anus; $\mathrm{avf}=$ advulval flap; $\mathrm{dgo}=$ pharyngeal dorsal gland orifice; lf = lateral field; $\mathrm{V}=$ vulva).

Molecular characterization: Five D2-D3 of 28S rRNA (MW798301-MW798305), four ITS (MW798343-MW798346) and two COI (MW797019-MW797020) gene sequences were generated for the first time from this species without intraspecific sequence variations, except for the ITS sequences (differing by 1 nucleotide and 2 indels). The closest Paratylenchus spp. was P. neoamblycephalus described in Singh et al. [24] with 99\% similarity for the D2-D3 of 
$28 \mathrm{~S}$ rRNA (differing by 7 nucleotides) to MW413660-MW413663, for the ITS sequences the similarity was $95 \%$ (differing by 45 nucleotides and 13 indels) with MW413606-MW413609, and finally sequences from COI gene regions showed a similarity of $90 \%$ (37 nucleotides) with the accession (MW421675-MW421682). However, morphologically and morphometrically $P$. israelensis and $P$. neoamblycephalus can be clearly separated by: labial framework (with strong sclerotization vs. light sclerotization), lip region shape (conical with protruding submedian lobes surrounding the oral aperture vs. conical-truncate with submedian lobes indistinct), and stylet length (24-26 $\mu \mathrm{m}$ vs. 26-34 $\mu \mathrm{m})$ [36].

Paratylenchus tenuicaudatus $\mathrm{Wu}, 1961$.

Described from Ontario, Canada in soil around roots of T. pratense, T. repens L., Acer saccharum Marsh, $M$. sativa, and grass sod. It has also been reported in several localities of USA $[77,80]$ and Iran [81]. The Iranian population of $P$. tenuicaudatus was also morphologically and molecularly similar to Paratylenchus sp. 1 from USA [23], suggesting that Paratylenchus sp. 1 from USA is conspecific with P. tenuicaudatus as mentioned by Esmaeili et al. [81].

The four Spanish populations of $P$. tenuicaudatus detected in our study agree with original description and others populations with molecular data available (Table 8, Figure 18). Only minor differences were found in all populations measured in a slightly shorter female body in comparison to the original description (354-392 $\mu \mathrm{m}, 307-407 \mu \mathrm{m}, 292-389 \mu \mathrm{m}$, and $394-414 \mu \mathrm{m}$ vs. $381-600 \mu \mathrm{m}$ ), but with a good size matching with the Iranian population (305-365 $\mu \mathrm{m}$ ); slightly posterior positon of the vulva in the Caravaca population (sample code PR_124) (82.1-84.7\% vs. 77.6-81.6\%) and longer female stylet in Sástago population (sample code PR_208) (32.0-33.5 $\mu \mathrm{m}$ vs. 25.1-31.5 $\mu \mathrm{m}$ ). Molecularly the Spanish populations are identical to the populations with molecular data available from Iran [81] and USA population (identified as Paratylenchus sp. 1) [23]. These four populations have been found in the rhizosphere of almond in Caravaca, Murcia province (sample code PR_124), peach in Calasparra, Murcia province (sample code PR_129), peach in Sollana, Valencia province (sample code PR_168) and apricot in Sástago, Zaragoza province (sample code PR_208) with 212, 10149, 15.050 and 12.950 individuals $/ 500 \mathrm{~cm}^{3}$ of soil, respectively. This species has been previously reported in Navarra forests (North Spain) by Hernández et al. [52], but no detailed morphology and measurements were provided.

Molecular characterization: Four D2-D3 of 28S rRNA (MW798306-MW798309), three ITS (MW798347-MW798349), and three COI gene sequences (MW797021-MW797023) were obtained from this species without intraspecific sequence variations. COI sequences were generated the first time in this study. D2-D3 of 28S rRNA sequences were shown to be $99 \%$ similar to the accession KU291239 (differing by 4 nucleotides) and ITS sequences were identical to the accession KF242260 [24,81].

Paratylenchus veruculatus Wu, 1962.

Described from soil around roots of heather from Kilmcolm, Scotland [82]. It has been also reported in Scotland [83], Belgium [24,83], Russia [73], Poland [33,75] and Iran [84].

The five Spanish populations of $P$. veruculatus agree with the original species description and other populations $[24,75,83,84]$, at exception of longer body in comparison to the original description (354-436 $\mu \mathrm{m}, 349-407 \mu \mathrm{m}, 303-445 \mu \mathrm{m}, 353-395 \mu \mathrm{m}, 279-441 \mu \mathrm{m}$ vs. $250-320 \mu \mathrm{m})$ and the other four populations of this species cited in the literature $(270 \mu \mathrm{m}$, 230-320 $\mu \mathrm{m}, 290-350 \mu \mathrm{m}$, and 251-331 $\mu \mathrm{m}$ ) (Table 9, Figure 19). Longer stylet in comparison to the original description $(14-16 \mu \mathrm{m}, 15.5-16.0 \mu \mathrm{m}, 14.5-16.5 \mu \mathrm{m}, 15.5-16.0 \mu \mathrm{m}$, $15.0-17.0 \mu \mathrm{m}$ vs. $12.0-15.0 \mu \mathrm{m})$ and others $(14 \mu \mathrm{m}, 10.5-14.5 \mu \mathrm{m}, 11.0-14.0 \mu \mathrm{m}$, and 13.1-14.8 $\mu \mathrm{m}$ ). However, all molecular markers of the Spanish populations agree with their identification as P. veruculatus with the molecular markers provided by Singh et al. [24]. These five populations have been found in almond in Puebla de Don Fadrique, Granada province (sample code PR_122), almond in Sta. $\mathrm{M}^{a}$ de Nieva, Almería province (sample code PR_106), almond in Lúcar, Almería province (sample code PR_115), almond in Serón, Almería province (sample code PR_118) and peach in La Almunia, Zaragoza province (sample code PR_193) with 180, 630, 1404, 1134 and 20 individuals $/ 500 \mathrm{~cm}^{3}$ of soil, respec- 
tively. This species has not been reported before in Spain and constitute a first record for the country.

Table 8. Morphometrics of Paratylenchus tenuicaudatus Wu, 1961 from several localities in Spain. All measurements are in $\mu \mathrm{m}$ and in the form: mean \pm s.d. (range).

\begin{tabular}{|c|c|c|c|c|c|}
\hline $\begin{array}{l}\text { Measurements } \\
\text { and Ratios }\end{array}$ & Females & Males & Females & Females & Females \\
\hline Sample code & PR_124 & PR_124 & PR_129 & PR_168 & PR_208 \\
\hline $\begin{array}{l}\text { Locality } \\
n\end{array}$ & $\begin{array}{c}\text { Caravaca, Murcia } \\
3\end{array}$ & $\begin{array}{c}\text { Caravaca, Murcia } \\
2\end{array}$ & $\begin{array}{c}\text { Calasparra, Murcia } \\
9\end{array}$ & $\begin{array}{c}\text { Sollana, Valencia } \\
4\end{array}$ & $\begin{array}{c}\text { Sástago, Zaragoza } \\
3\end{array}$ \\
\hline $\mathrm{L}$ & $\begin{array}{c}368.7 \pm 20.4 \\
(354-392)\end{array}$ & 356,364 & $\begin{array}{c}376.6 \pm 31.0 \\
(307-407)\end{array}$ & $\begin{array}{c}358.5 \pm 44.7 \\
(292-389)\end{array}$ & $\begin{array}{c}405.3 \pm 10.3 \\
(394-414)\end{array}$ \\
\hline$a^{*}$ & $\begin{array}{l}23.5 \pm 1.3 \\
(22.1-24.5)\end{array}$ & $29.1,29.7$ & $\begin{array}{l}22.7 \pm 3.7 \\
(16.5-28.4)\end{array}$ & $\begin{array}{l}23.6 \pm 2.0 \\
(20.9-25.1)\end{array}$ & $\begin{array}{l}18.3 \pm 0.8 \\
(17.4-18.8)\end{array}$ \\
\hline $\mathrm{b}$ & $\begin{array}{l}4.5 \pm 0.2 \\
(4.2-4.6)\end{array}$ & $4.4,4.7$ & $\begin{array}{l}3.8 \pm 0.3 \\
(3.4-4.2)\end{array}$ & $\begin{array}{l}4.0 \pm 0.4 \\
(3.4-4.2)\end{array}$ & $4.0 \pm 0.0$ \\
\hline c & $\begin{array}{c}11.7 \pm 1.9 \\
(10.4-13.8)\end{array}$ & $11.1,12.3$ & $\begin{array}{c}11.7 \pm 1.3 \\
(10.5-14.4)\end{array}$ & $\begin{array}{l}9.7 \pm 0.9 \\
(8.7-10.5)\end{array}$ & $\begin{array}{c}10.3 \pm 0.5 \\
(10.0-10.9)\end{array}$ \\
\hline$c^{\prime}$ & $\begin{array}{l}3.7 \pm 0.4 \\
(3.3-4.0)\end{array}$ & $3.0,3.8$ & $\begin{array}{l}3.6 \pm 0.5 \\
(2.8-4.8)\end{array}$ & $\begin{array}{l}3.8 \pm 0.5 \\
(3.3-4.2)\end{array}$ & $\begin{array}{l}3.4 \pm 0.1 \\
(3.3-3.5)\end{array}$ \\
\hline $\mathrm{V}$ or $\mathrm{T}$ & $\begin{array}{c}83.0 \pm 1.5 \\
(82.1-84.7)\end{array}$ & $39.3,46.3$ & $\begin{array}{l}80.8 \pm 1.3 \\
(78.3-82.5)\end{array}$ & $\begin{array}{l}80.6 \pm 0.8 \\
(79.6-81.5)\end{array}$ & $\begin{array}{l}80.2 \pm 0.8 \\
(79.2-80.7)\end{array}$ \\
\hline G1 & $\begin{array}{l}46.3 \pm 10.8 \\
(33.9-52.8)\end{array}$ & - & $\begin{array}{l}41.3 \pm 7.4 \\
(27.6-50.4)\end{array}$ & $\begin{array}{l}34.8 \pm 5.5 \\
(29.8-41.2)\end{array}$ & $\begin{array}{l}43.7 \pm 3.3 \\
(40.1-46.6)\end{array}$ \\
\hline Stylet length & $\begin{array}{l}29.3 \pm 0.6 \\
(29.0-30.0)\end{array}$ & - & $\begin{array}{l}30.7 \pm 1.1 \\
(29.0-32.0)\end{array}$ & $\begin{array}{l}30.5 \pm 1.5 \\
(29.0-32.0)\end{array}$ & $\begin{array}{l}32.7 \pm 0.8 \\
(32.0-33.5)\end{array}$ \\
\hline Conus length & $\begin{array}{c}17.7 \pm 0.6 \\
(17.0-18.0)\end{array}$ & - & $\begin{array}{l}21.0 \pm 1.2 \\
(19.5-23.0)\end{array}$ & $\begin{array}{l}20.5 \pm 1.9 \\
(18.0-22.0)\end{array}$ & $\begin{array}{l}21.7 \pm 1.2 \\
(21.0-23.0)\end{array}$ \\
\hline $\mathrm{m}$ & $\begin{array}{c}60.9 \pm 2.0 \\
(58.6-62.1)\end{array}$ & - & $\begin{array}{l}68.4 \pm 1.7 \\
(66.7-71.9)\end{array}$ & $\begin{array}{c}67.1 \pm 4.1 \\
(61.0-69.8)\end{array}$ & $\begin{array}{c}66.3 \pm 2.1 \\
(64.6-68.7)\end{array}$ \\
\hline DGO & $\begin{array}{l}5.3 \pm 0.6 \\
(5.0-6.0)\end{array}$ & - & $\begin{array}{l}5.0 \pm 0.9 \\
(4.0-6.5)\end{array}$ & $\begin{array}{l}6.7 \pm 0.6 \\
(6.0-7.0)\end{array}$ & $\begin{array}{l}7.2 \pm 0.3 \\
(7.0-7.5)\end{array}$ \\
\hline $\mathrm{O}$ & $\begin{array}{l}18.4 \pm 2.0 \\
(17.2-20.7)\end{array}$ & - & $\begin{array}{l}16.3 \pm 2.7 \\
(12.7-20.3)\end{array}$ & $\begin{array}{l}22.2 \pm 1.5 \\
(20.7-23.7)\end{array}$ & $\begin{array}{l}21.9 \pm 0.4 \\
(21.5-22.4)\end{array}$ \\
\hline Lip width & $\begin{array}{l}6.2 \pm 0.3 \\
(6.0-6.5)\end{array}$ & 4.0 & $\begin{array}{l}4.9 \pm 0.8 \\
(4.0-6.5)\end{array}$ & $\begin{array}{l}5.3 \pm 0.5 \\
(5.0-6.0)\end{array}$ & $\begin{array}{l}7.2 \pm 0.3 \\
(7.0-7.5)\end{array}$ \\
\hline Median bulb length & (10 & - & $\begin{array}{l}23.4 \pm 2.9 \\
(18.0-27.0)\end{array}$ & $\begin{array}{c}17.5 \pm 2.1 \\
(16.0-19.0)\end{array}$ & $\begin{array}{l}28.0 \pm 1.0 \\
(27.0-29.0)\end{array}$ \\
\hline Median bulb width & - & - & $\begin{array}{l}9.1 \pm 1.4 \\
(7.0-11.5)\end{array}$ & $\begin{array}{l}8.8 \pm 1.3 \\
(7.5-10.0)\end{array}$ & $\begin{array}{c}12.8 \pm 0.8 \\
(12.0-13.5)\end{array}$ \\
\hline $\begin{array}{l}\text { Anterior end to } \\
\text { center median bulb }\end{array}$ & $\begin{array}{c}44.7 \pm 1.2 \\
(44.0-46.0)\end{array}$ & $44.0,46.0$ & $\begin{array}{l}57.9 \pm 3.6 \\
(52.0-63.0)\end{array}$ & $\begin{array}{l}50.8 \pm 3.2 \\
(49.0-54.5)\end{array}$ & $\begin{array}{l}61.0 \pm 1.0 \\
(60.0-62.0)\end{array}$ \\
\hline (2) & $\begin{array}{l}54.3 \pm 2.4 \\
(51.8-56.4)\end{array}$ & $56.1,58.7$ & $\begin{array}{l}57.7 \pm 3.4 \\
(54.4-64.2)\end{array}$ & $\begin{array}{l}56.2 \pm 3.2 \\
(52.7-58.9)\end{array}$ & $\begin{array}{c}60.4 \pm 0.2 \\
(60.2-60.6)\end{array}$ \\
\hline $\begin{array}{l}\text { Nerve ring to } \\
\text { anterior end }\end{array}$ & $\begin{array}{l}61.7 \pm 4.5 \\
(57.0-66.0)\end{array}$ & $54.0,55.0$ & $\begin{array}{l}73.9 \pm 5.3 \\
(66.5-81.0)\end{array}$ & $\begin{array}{l}64.0 \pm 6.2 \\
(57.0-69.0)\end{array}$ & $\begin{array}{l}80.3 \pm 0.8 \\
(79.2-80.7)\end{array}$ \\
\hline $\begin{array}{l}\text { Excretory pore to } \\
\text { anterior end }\end{array}$ & $\begin{array}{l}75.3 \pm 2.3 \\
(74.0-78.0)\end{array}$ & $59.0,71.0$ & $\begin{array}{l}80.4 \pm 7.7 \\
(68.0-88.0)\end{array}$ & $\begin{array}{l}75.2 \pm 2.4 \\
(72.5-77.0)\end{array}$ & $\begin{array}{c}93.3 \pm 7.2 \\
(85.0-98.0)\end{array}$ \\
\hline Pharynx length & $\begin{array}{l}82.3 \pm 3.8 \\
(78.0-85.0)\end{array}$ & $75.0,82.0$ & $\begin{array}{c}98.1 \pm 7.6 \\
(88.0-110.0)\end{array}$ & $\begin{array}{l}90.4 \pm 3.2 \\
(86.0-93.0)\end{array}$ & $\begin{array}{l}101.0 \pm 2.0 \\
(99.0-103.0)\end{array}$ \\
\hline $\begin{array}{l}\text { Maximum body } \\
\text { diam. }\end{array}$ & $\begin{array}{c}15.7 \pm 0.6 \\
(15.0-16.0)\end{array}$ & $12.0,12.5$ & $\begin{array}{l}17.1 \pm 3.8 \\
(12.0-24.0)\end{array}$ & $\begin{array}{l}15.1 \pm 0.9 \\
(14.0-16.0)\end{array}$ & $\begin{array}{l}22.2 \pm 1.3 \\
(21.0-23.5)\end{array}$ \\
\hline Tail length & $\begin{array}{l}32.0 \pm 5.3 \\
(26.0-36.0)\end{array}$ & $29.5,32.0$ & $\begin{array}{l}32.6 \pm 4.8 \\
(25.0-38.0)\end{array}$ & $\begin{array}{l}37.3 \pm 6.9 \\
(28.0-43.0)\end{array}$ & $\begin{array}{l}39.3 \pm 1.5 \\
(38.0-41.0)\end{array}$ \\
\hline Anal body diam. & $\begin{array}{l}8.7 \pm 0.6 \\
(8.0-9.0)\end{array}$ & $8.5,10.0$ & $\begin{array}{l}9.0 \pm 1.2 \\
(7.0-11.0)\end{array}$ & $\begin{array}{l}9.8 \pm 1.7 \\
(8.5-12.0)\end{array}$ & $\begin{array}{l}11.7 \pm 0.6 \\
(11.0-12.0)\end{array}$ \\
\hline Spicules & $\begin{array}{c}(0.0-1.0) \\
-\end{array}$ & $22.0,25.0$ & $\begin{array}{c}(1.0-110) \\
-\end{array}$ & $\begin{array}{c}(0.0-120) \\
-\end{array}$ & $\begin{array}{c}(11.0-12.0) \\
-\end{array}$ \\
\hline Gubernaculum & - & 6.0, 7.5 & - & - & - \\
\hline
\end{tabular}

* Abbreviations: $\mathrm{a}=$ body length/greatest body diameter; $\mathrm{b}=$ body length/distance from anterior end to pharyngo-intestinal junction; $\mathrm{DGO}=$ distance between stylet base and orifice of dorsal pharyngeal gland; $\mathrm{c}=$ body length $/$ tail length; $\mathrm{c}^{\prime}=$ tail length $/$ tail diameter at anus or cloaca; G1 = anterior genital branch length expressed as percentage $(\%)$ of the body length; $\mathrm{L}=$ overall body length; $\mathrm{m}=$ length of conus as percentage of total stylet length; $\mathrm{MB}=$ distance between anterior end of body and center of median pharyngeal bulb expressed as percentage $(\%)$ of the pharynx length; $\mathrm{n}=$ number of specimens on which measurements are based; $\mathrm{O}=\mathrm{DGO}$ as percentage of stylet length; $\mathrm{T}=$ distance from cloacal aperture to anterior end of testis expressed as percentage $(\%)$ of the body length; $\mathrm{V}=$ distance from body anterior end to vulva expressed as percentage (\%) of the body length. 


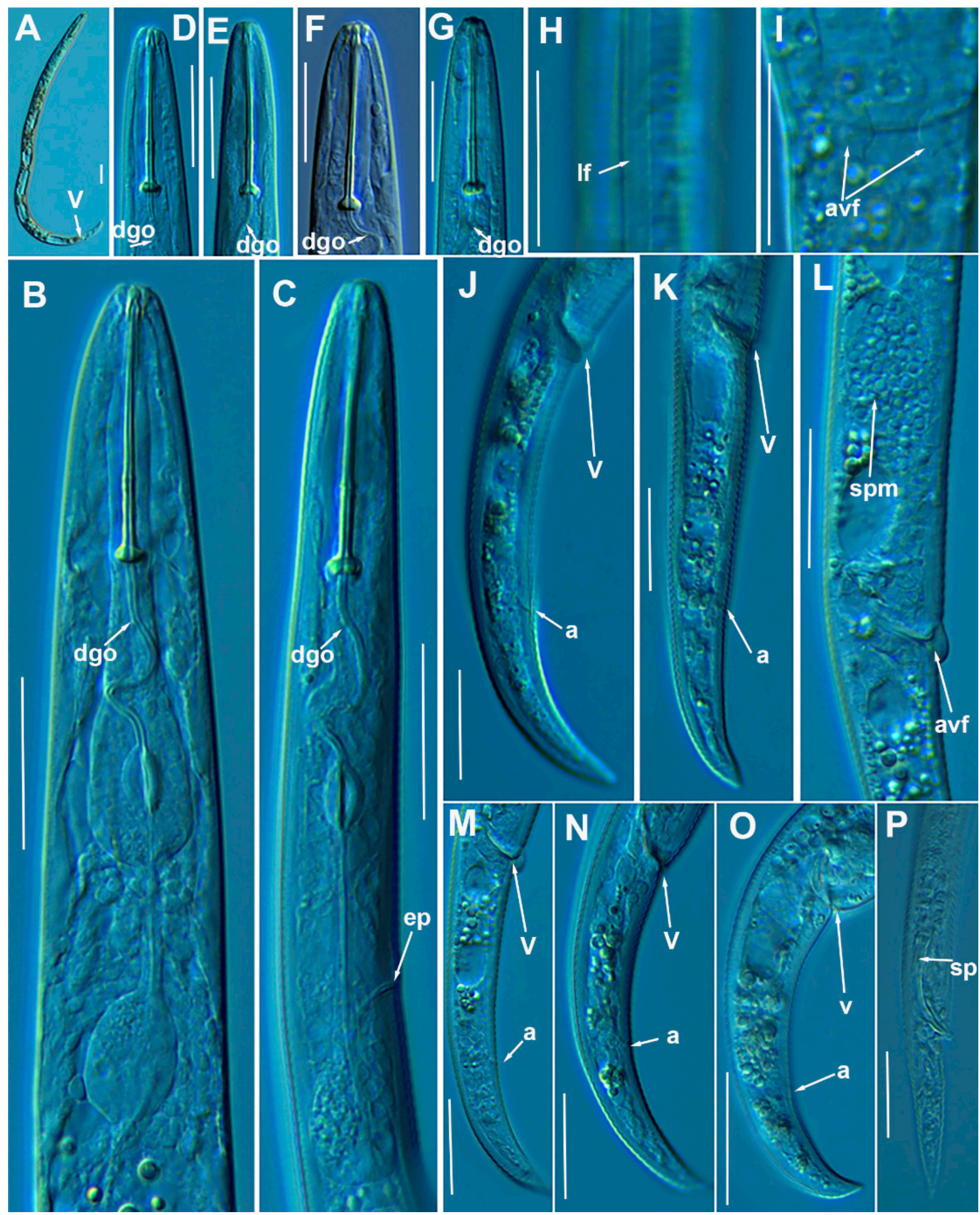

Figure 18. Light photomicrographs of Paratylenchus tenuicaudatus Wu, 1961. (A): Entire female with vulva arrowed; (B,C): Female pharyngeal region; (D-G): Female lip region; (H): Detail of female lateral field at mid-body (arrowed); (I): Detail of vulva showing a frontal view of advulval flap (arrowed); (J-O): Female posterior region showing vulva and anus (arrowed); (P): Male tail with spicules arrowed. Scale bars $(\mathbf{A}-\mathbf{P}=20 \mu \mathrm{m})$. (Abbreviations: a = anus; avf = advulval flap; $\mathrm{dgo}=$ pharyngeal dorsal gland orifice; $\mathrm{ep}=$ excretory pore; $\mathrm{lf}=$ lateral field; $\mathrm{spm}=$ spermatheca; $\mathrm{sp}=\mathrm{spicules} ; \mathrm{V}=\mathrm{vulva}$ ) 
Table 9. Morphometrics of Paratylenchus veruculatus $\mathrm{Wu}, 1962$ from several localities in Spain. All measurements are in $\mu \mathrm{m}$ and in the form: mean \pm s.d. (range).

\begin{tabular}{|c|c|c|c|c|c|}
\hline $\begin{array}{c}\text { Measurements } \\
\text { and Ratios }\end{array}$ & Females & Females & Females & Females & Females \\
\hline Sample code & PR_122 & PR_106 & PR_115 & PR_118 & PR_193 \\
\hline Locality & $\begin{array}{l}\text { Puebla de Don } \\
\text { Fadrique, Granada }\end{array}$ & $\begin{array}{l}\text { Sta. Ma Nieva, } \\
\text { Almería }\end{array}$ & Lúcar, Almería & Serón, Almería & $\begin{array}{c}\text { La Almunia, } \\
\text { Zaragoza }\end{array}$ \\
\hline $\mathrm{n}$ & 10 & 4 & 10 & 4 & 10 \\
\hline $\mathrm{L}$ & $\begin{array}{c}376.1 \pm 30.4 \\
(354-436)\end{array}$ & $\begin{array}{c}381.5 \pm 25.4 \\
(349-407)\end{array}$ & $\begin{array}{c}374.5 \pm 37.8 \\
(303-445)\end{array}$ & $\begin{array}{c}379.5 \pm 19.2 \\
(353-395)\end{array}$ & $\begin{array}{c}363.8 \pm 45.8 \\
(279-441)\end{array}$ \\
\hline$a^{*}$ & $\begin{array}{c}20.4 \pm 2.5 \\
(17.0-24.3)\end{array}$ & $\begin{array}{c}20.7 \pm 0.3 \\
(17.5-24.7)\end{array}$ & $\begin{array}{c}20.4 \pm 1.9 \\
(16.8-23.9)\end{array}$ & $\begin{array}{c}22.2 \pm 0.7 \\
(21.4-23.1)\end{array}$ & $\begin{array}{c}19.8 \pm 1.6 \\
(16.4-21.7)\end{array}$ \\
\hline $\mathrm{b}$ & $\begin{array}{l}4.0 \pm 0.2 \\
(3.7-4.4)\end{array}$ & $\begin{array}{l}4.2 \pm 0.4 \\
(3.8-4.8)\end{array}$ & $\begin{array}{l}4.0 \pm 0.4 \\
(3.4-4.7)\end{array}$ & $\begin{array}{l}4.1 \pm 0.3 \\
(3.8-4.4)\end{array}$ & $\begin{array}{l}3.8 \pm 0.3 \\
(3.4-4.4)\end{array}$ \\
\hline c & $\begin{array}{l}14.8 \pm 2.0 \\
(11.8-19.7)\end{array}$ & $\begin{array}{c}12.9 \pm 0.6 \\
(12.0-13.4)\end{array}$ & $\begin{array}{l}15.5 \pm 1.7 \\
(13.1-18.4)\end{array}$ & $\begin{array}{c}13.7 \pm 0.3 \\
(13.5-14.1)\end{array}$ & $\begin{array}{c}14.0-1.5 \\
(11.2-15.8)\end{array}$ \\
\hline$c^{\prime}$ & $\begin{array}{l}2.5 \pm 0.2 \\
(2.3-2.8)\end{array}$ & $\begin{array}{l}2.7 \pm 0.3 \\
(2.3-2.9)\end{array}$ & $\begin{array}{l}2.4 \pm 0.3 \\
(2.1-2.8)\end{array}$ & $\begin{array}{c}2.8 \pm 0.04 \\
(2.7-2.8)\end{array}$ & $\begin{array}{l}2.8 \pm 0.2 \\
(2.5-3.0)\end{array}$ \\
\hline $\mathrm{V}$ & $\begin{array}{l}84.2 \pm 1.0 \\
(82.7-85.5)\end{array}$ & $\begin{array}{c}84.0 \pm 0.9 \\
(83.1-84.8)\end{array}$ & $\begin{array}{l}83.6 \pm 0.6 \\
(82.8-84.5)\end{array}$ & $\begin{array}{l}83.8 \pm 1.1 \\
(82.8-84.9)\end{array}$ & $\begin{array}{l}84.3 \pm 0.8 \\
(83.1-85.4)\end{array}$ \\
\hline G1 & $\begin{array}{l}36.5 \pm 7.3 \\
(22.3-47.7)\end{array}$ & $\begin{array}{l}34.2 \pm 12.5 \\
(26.7-52.9)\end{array}$ & $\begin{array}{l}38.5 \pm 4.1 \\
(32.0-43.2)\end{array}$ & $\begin{array}{l}33.6 \pm 0.8 \\
(25.9-43.6)\end{array}$ & $\begin{array}{l}35.6 \pm 4.7 \\
(29.8-44.4)\end{array}$ \\
\hline Stylet length & $\begin{array}{c}15.1 \pm 0.6 \\
(14.0-16.0)\end{array}$ & $\begin{array}{l}15.9 \pm 0.3 \\
(15.5-16.0)\end{array}$ & $\begin{array}{l}15.5 \pm 0.6 \\
(14.5-16.5)\end{array}$ & $\begin{array}{l}15.6 \pm 0.25 \\
(15.5-16.0)\end{array}$ & $\begin{array}{l}15.7 \pm 0.7 \\
(15.0-17.0)\end{array}$ \\
\hline Conus length & $\begin{array}{l}9.1 \pm 0.7 \\
(7.5-10.0)\end{array}$ & $\begin{array}{l}9.9 \pm 0.3 \\
(9.5-10.0)\end{array}$ & $\begin{array}{l}9.5 \pm 0.6 \\
(8.0-10.0)\end{array}$ & $\begin{array}{l}9.8 \pm 0.3 \\
(9.5-10.0)\end{array}$ & $\begin{array}{c}9.6 \pm 0.5 \\
(9.0-10.5)\end{array}$ \\
\hline $\mathrm{m}$ & $\begin{array}{l}60.4 \pm 5.5 \\
(50.0-66.7)\end{array}$ & $\begin{array}{l}62.2 \pm 0.6 \\
(61.3-62.5)\end{array}$ & $\begin{array}{l}60.9 \pm 2.3 \\
(55.2-63.3)\end{array}$ & $\begin{array}{l}61.4 \pm 2.5 \\
(59.4-64.5)\end{array}$ & $\begin{array}{l}61.3 \pm 1.8 \\
(58.1-63.3)\end{array}$ \\
\hline DGO & $\begin{array}{l}5.2 \pm 1.2 \\
(3.0-7.0)\end{array}$ & $\begin{array}{l}4.3 \pm 0.3 \\
(4.0-4.5)\end{array}$ & $\begin{array}{l}4.5 \pm 0.6 \\
(3.5-5.5)\end{array}$ & $\begin{array}{l}3.6 \pm 0.6 \\
(3.0-4.5)\end{array}$ & $\begin{array}{l}4.5 \pm 0.4 \\
(4.0-5.0)\end{array}$ \\
\hline $\mathrm{O}$ & $\begin{array}{l}34.3 \pm 7.4 \\
(20.0-46.7)\end{array}$ & $\begin{array}{l}26.8 \pm 2.1 \\
(25.0-29.0)\end{array}$ & $\begin{array}{l}28.7 \pm 3.3 \\
(21.9-33.3)\end{array}$ & $\begin{array}{l}22.8 \pm 3.9 \\
(18.8-28.1)\end{array}$ & $\begin{array}{l}28.8 \pm 2.7 \\
(25.0-33.3)\end{array}$ \\
\hline Lip width & $\begin{array}{l}6.6 \pm 0.6 \\
(6.0-7.5)\end{array}$ & $\begin{array}{l}6.0 \pm 0.4 \\
(5.5-6.5)\end{array}$ & $\begin{array}{l}6.3 \pm 0.4 \\
(6.0-7.0)\end{array}$ & $\begin{array}{l}7.0 \pm 0.4 \\
(6.5-7.5)\end{array}$ & $\begin{array}{l}6.7 \pm 0.4 \\
(6.0-7.5)\end{array}$ \\
\hline $\begin{array}{l}\text { Median bulb } \\
\text { length }\end{array}$ & $\begin{array}{l}23.8 \pm 2.4 \\
(22.0-27.0)\end{array}$ & $\begin{array}{l}25.3 \pm 1.0 \\
(24.0-26.0)\end{array}$ & $\begin{array}{l}24.6 \pm 5.0 \\
(19.0-33.0)\end{array}$ & $\begin{array}{l}26.5 \pm 0.7 \\
(26.0-27.0)\end{array}$ & $\begin{array}{l}20.8 \pm 1.5 \\
(19.0-22.0)\end{array}$ \\
\hline Median bulb width & $\begin{array}{l}8.1 \pm 0.6 \\
(7.5-9.0)\end{array}$ & $\begin{array}{l}8.4 \pm 0.5 \\
(8.0-9.0)\end{array}$ & $\begin{array}{l}9.3 \pm 1.3 \\
(8.0-12.0)\end{array}$ & $\begin{array}{l}9.5 \pm 0.7 \\
(9.0-10.0)\end{array}$ & $\begin{array}{l}9.3 \pm 1.2 \\
(8.0-11.0)\end{array}$ \\
\hline $\begin{array}{l}\text { Anterior end to } \\
\text { center median bulb }\end{array}$ & $\begin{array}{l}49.3 \pm 2.8 \\
(44.0-53.0)\end{array}$ & $\begin{array}{l}47.5 \pm 2.4 \\
(45.0-50.0)\end{array}$ & $\begin{array}{l}48.0 \pm 2.1 \\
(45.0-51.0)\end{array}$ & $\begin{array}{l}49.8 \pm 2.1 \\
(47.0-52.0)\end{array}$ & $\begin{array}{l}49.0 \pm 3.4 \\
(43.0-54.0)\end{array}$ \\
\hline $\mathrm{MB}$ & $\begin{array}{c}51.2 \pm 2.2 \\
(47.8-54.1)\end{array}$ & $\begin{array}{l}52.4 \pm 1.3 \\
(51.1-53.8)\end{array}$ & $\begin{array}{l}51.6 \pm 1.5 \\
(48.4-53.4)\end{array}$ & $\begin{array}{l}53.2 \pm 3.8 \\
(50.5-58.8)\end{array}$ & $\begin{array}{l}50.0 \pm 2.7 \\
(46.7-53.8)\end{array}$ \\
\hline $\begin{array}{l}\text { Nerve ring to } \\
\text { anterior end }\end{array}$ & $\begin{array}{l}68.6 \pm 6.5 \\
(60.0-80.0)\end{array}$ & $\begin{array}{l}65.0 \pm 4.4 \\
(60.0-70.0)\end{array}$ & $\begin{array}{l}65.1 \pm 4.1 \\
(59.0-70.0)\end{array}$ & $\begin{array}{l}69.5 \pm 4.4 \\
(64.0-74.0)\end{array}$ & $\begin{array}{l}68.3 \pm 5.3 \\
(61.0-76.0)\end{array}$ \\
\hline $\begin{array}{l}\text { Excretory pore to } \\
\text { anterior end }\end{array}$ & $\begin{array}{l}82.6 \pm 8.1 \\
(69.0-97.0)\end{array}$ & $\begin{array}{l}85.5 \pm 6.6 \\
(79.0-93.0)\end{array}$ & $\begin{array}{l}81.0 \pm 6.3 \\
(70.0-92.0)\end{array}$ & $\begin{array}{l}84.3 \pm 6.7 \\
(75.0-90.0)\end{array}$ & $\begin{array}{l}84.0 \pm 7.3 \\
(75.0-97.0)\end{array}$ \\
\hline Pharynx length & $\begin{array}{c}94.8 \pm 7.1 \\
(84.0-108.0)\end{array}$ & $\begin{array}{l}90.8 \pm 4.9 \\
(85.0-97.0)\end{array}$ & $\begin{array}{c}93.0 \pm 3.8 \\
(88.0-101.0)\end{array}$ & $\begin{array}{c}94.0 \pm 9.4 \\
(80.0-100.0)\end{array}$ & $\begin{array}{c}95.4 \pm 7.0 \\
(81.0-105.0)\end{array}$ \\
\hline $\begin{array}{l}\text { Maximum body } \\
\text { diam. }\end{array}$ & $\begin{array}{l}18.7 \pm 2.7 \\
(15.0-23.0)\end{array}$ & $\begin{array}{l}18.6 \pm 1.5 \\
(16.5-20.0)\end{array}$ & $\begin{array}{l}18.4 \pm 1.7 \\
(15.0-20.0)\end{array}$ & $\begin{array}{c}17.1 \pm 0.6 \\
(16.5-18.0)\end{array}$ & $\begin{array}{c}18.5 \pm 2.3 \\
(16.5-24.0)\end{array}$ \\
\hline Tail length & $\begin{array}{l}25.9 \pm 4.6 \\
(18.0-36.5)\end{array}$ & $\begin{array}{l}29.6 \pm 1.3 \\
(28.5-31.5)\end{array}$ & $\begin{array}{l}24.5 \pm 4.0 \\
(16.5-30.0)\end{array}$ & $\begin{array}{l}27.6 \pm 1.1 \\
(26.0-28.5)\end{array}$ & $\begin{array}{l}26.1 \pm 1.7 \\
(23.0-28.0)\end{array}$ \\
\hline Anal body diam. & $\begin{array}{l}10.4 \pm 1.4 \\
(8.0-13.0)\end{array}$ & $\begin{array}{l}11.3 \pm 1.6 \\
(10.0-13.5)\end{array}$ & $\begin{array}{l}10.0 \pm 0.9 \\
(8.0-11.0)\end{array}$ & $\begin{array}{l}10.0 \pm 0.4 \\
(9.5-10.5)\end{array}$ & $\begin{array}{l}9.4 \pm 0.8 \\
(8.0-11.0)\end{array}$ \\
\hline
\end{tabular}

* Abbreviations: $\mathrm{a}=$ body length/greatest body diameter; $\mathrm{b}=$ body length/distance from anterior end to pharyngo-intestinal junction; $\mathrm{DGO}=$ distance between stylet base and orifice of dorsal pharyngeal gland; $\mathrm{c}=$ body length/tail length; $\mathrm{c}^{\prime}=$ tail length/tail diameter at anus or cloaca; G1 = anterior genital branch length expressed as percentage (\%) of the body length; $\mathrm{L}=$ overall body length; $\mathrm{m}=$ length of conus as percentage of total stylet length; $\mathrm{MB}=$ distance between anterior end of body and center of median pharyngeal bulb expressed as percentage (\%) of the pharynx length; $\mathrm{n}=$ number of specimens on which measurements are based; $\mathrm{O}=\mathrm{DGO}$ as percentage of stylet length; $\mathrm{T}=$ distance from cloacal aperture to anterior end of testis expressed as percentage $(\%)$ of the body length; $\mathrm{V}=$ distance from body anterior end to vulva expressed as percentage (\%) of the body length. 

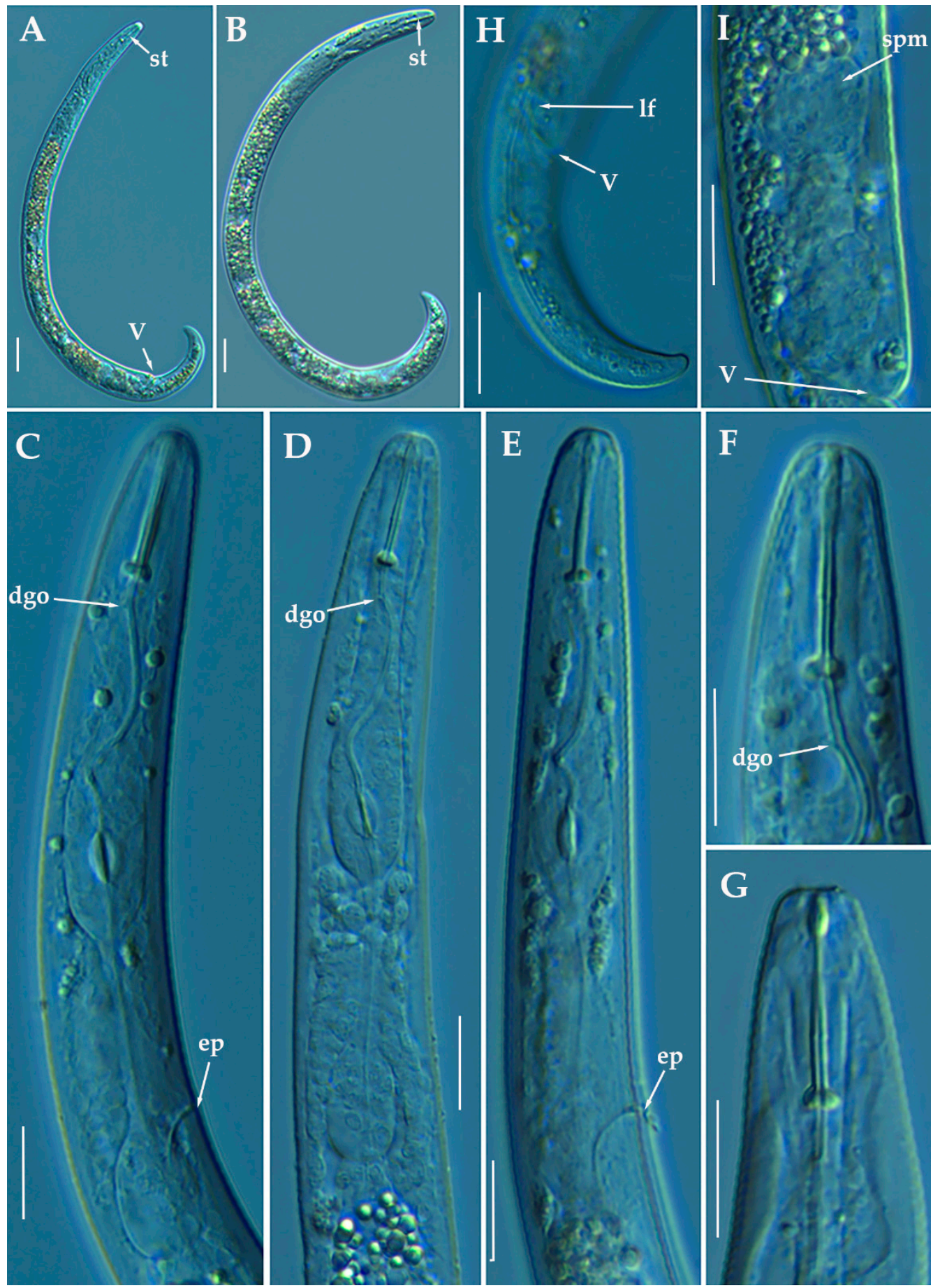

Figure 19. Light photomicrographs of female of Paratylenchus veruculatus Wu, 1962. (A): Entire female with stylet and vulva arrowed; (B): Fourth-stage juvenile with stylet arrowed; (C-E): Female pharyngeal region; (F-G): Female lip region; $(\mathbf{H})$ : Female posterior region with lateral field and vulva arrowed; (I): Detail of empty spermatheca (arrowed). Scale bars $(\mathbf{A}, \mathbf{B}, \mathbf{H}=20 \mu \mathrm{m} ; \mathbf{C}-\mathbf{G}, \mathbf{I}=10 \mu \mathrm{m})$. (Abbreviations: $\mathrm{a}$ = anus; $\mathrm{dgo}=$ pharyngeal dorsal gland orifice; $\mathrm{ep}=$ excretory pore; If = lateral field; spm = spermatheca; st $=$ stylet; $\mathrm{V}=$ vulva) .

Molecular characterization: Six D2-D3 of 28S rRNA (MW798310-MW798315) with a intraspecific sequence variation of $2 \%$ (differing from 0 to 13 nucleotides and 1 indel), five ITS (MW798350-MW798354) (96\% similarity; 27 nucleotides and 6 indels), and finally, six COI gene sequences (MW797024-MW797029) with a intraspecific sequence variation of 5\% 
(differing from 0 to 19 nucleotides). The D2-D3 of 28S rRNA sequences matched well with other accession of P. veruculatus deposited in GenBank showing similarity values from 98 to $99 \%$ (differing from 1 to 10 nucleotides and 1 indel) with MW413687, and from 94 to $95 \%$ for the COI gene sequences (differing from 19 to 21 nucleotides) with MW421717 [24]. ITS sequences (MW798350-MW798354) from P. veruculatus were generated for the first time in this study.

\subsection{Phylogenetic Analyses}

The D2-D3 domains of the 28S rRNA gene alignment (699 bp long) included 98 sequences of 54 Paratylenchus species and three outgroup species [Basiria gracillis (DQ328717), Aglenchus agricola (AY780979), and Coslenchus costatus (DQ328719)]. Forty-six new sequences were included in this analysis. The Bayesian 50\% majority rule consensus tree inferred from the D2-D3 alignment is given in Figure 20. The tree contained one highly supported major clade $(\mathrm{PP}=1.0)$ and two moderately supported clades $(\mathrm{PP}=0.92, \mathrm{PP}=0.92)$ ). These clades are mainly coincident with other recent studies on Paratylenchus spp. [24,28].

The ITS rRNA gene alignment (778 bp long) included 84 sequences of 48 Paratylenchus species and three outgroup species [Hemicriconemoides californianus (KF856557), Hemicriconemoides alexis (KF856562) and Hemicycliophora poranga (KF430598)]. Thirty-seven new sequences were included in this analysis. The Bayesian $50 \%$ majority rule consensus tree inferred from the ITS alignment is given in Figure 21. The tree contained one highly supported major clade $(\mathrm{PP}=1.00)$ and other clade not supported (but highly supported if excluding P. idalimus (KF242275)). These clades were partially coincident with previous studies with in some case similar or different clade support $[24,28]$.

The COI gene alignment ( $340 \mathrm{bp}$ long) included 161 sequences of 34 Paratylenchus species and three outgroup species [Hemicriconemoides californianus (KM516192), Hemicycliophora floridensis (MG019867) and Hemicycliophora poranga (MG019892)]. Seventy-one new sequences were included in this analysis. The Bayesian $50 \%$ majority rule consensus tree inferred from the COI sequence alignment is given in Figure 22. The tree contained one highly supported major clade $(\mathrm{PP}=1.00)$ and one moderately supported clade $(\mathrm{PP}=0.75)$. These clades were partially coincident with other studies with in some case similar or different clade support [24].

Paratylenchus caravaquenus sp. nov. is closely related phylogenetically in the D2-D3 domains of the $28 \mathrm{~S}$ rRNA to $P$. nawadus (MN088373) in a high supported clade ( $\mathrm{PP}=1.00)$. These two species are related in a larger clade to Paratylenchus sp. 4 SAS-2014 (KF242203), P. tateae (MW282756 and MW282758), P. projectus (MW413656), P. neoamblycephalus A USA (MG925221), P. neoprojectus (MW282762), P. nanus (KF242200) and P. coronatus (MK506808) $(\mathrm{PP}=1.00)$. For ITS region, P. caravaquenus sp. nov. is related with P. projectus (KF242266), P. nanus (MH236098) and P. coronatus (MK506795) in a high supported clade (PP =1.00) (Figure 21). While for COI marker, this species was separated in a clade, but their phylogenetic relationship was not clearly defined with other species for this marker (Figure 22).

Paratylenchus indalus sp. nov. was closely related in all markers studied (D2-D3 domains of the 28S rRNA, ITS and COI) with P. enigmaticus with high-supported clades (PP $=0.98-1.00)$ (Figures 20-22).

Paratylenchus pedrami sp. nov. was closely related in all markers studied (D2-D3 domains of the $28 \mathrm{~S}$ rRNA, ITS and COI) with P. baldaccii with high-supported clades $(\mathrm{PP}=1.00)$ (Figures 20-22).

Paratylenchus zurgenerus sp. nov. was closely related to Paratylenchus sp. 7 SAS-2014 (KF242242) in D2-D3 tree (most probably is conspecific with P. zurgenerus sp. nov.) and to $P$. microdorus (MW413654) and an undescribed species Paratylenchus sp. BE11 (MW413672), and in ITS tree also related to P. microdorus (MW413597) and an undescribed species Paratylenchus sp. BE11 (MW413617) (Figures 20 and 21). COI marker did not show a clear relationship with other species, but this species was clearly separated from the other species in a unique clade (Figure 22). 


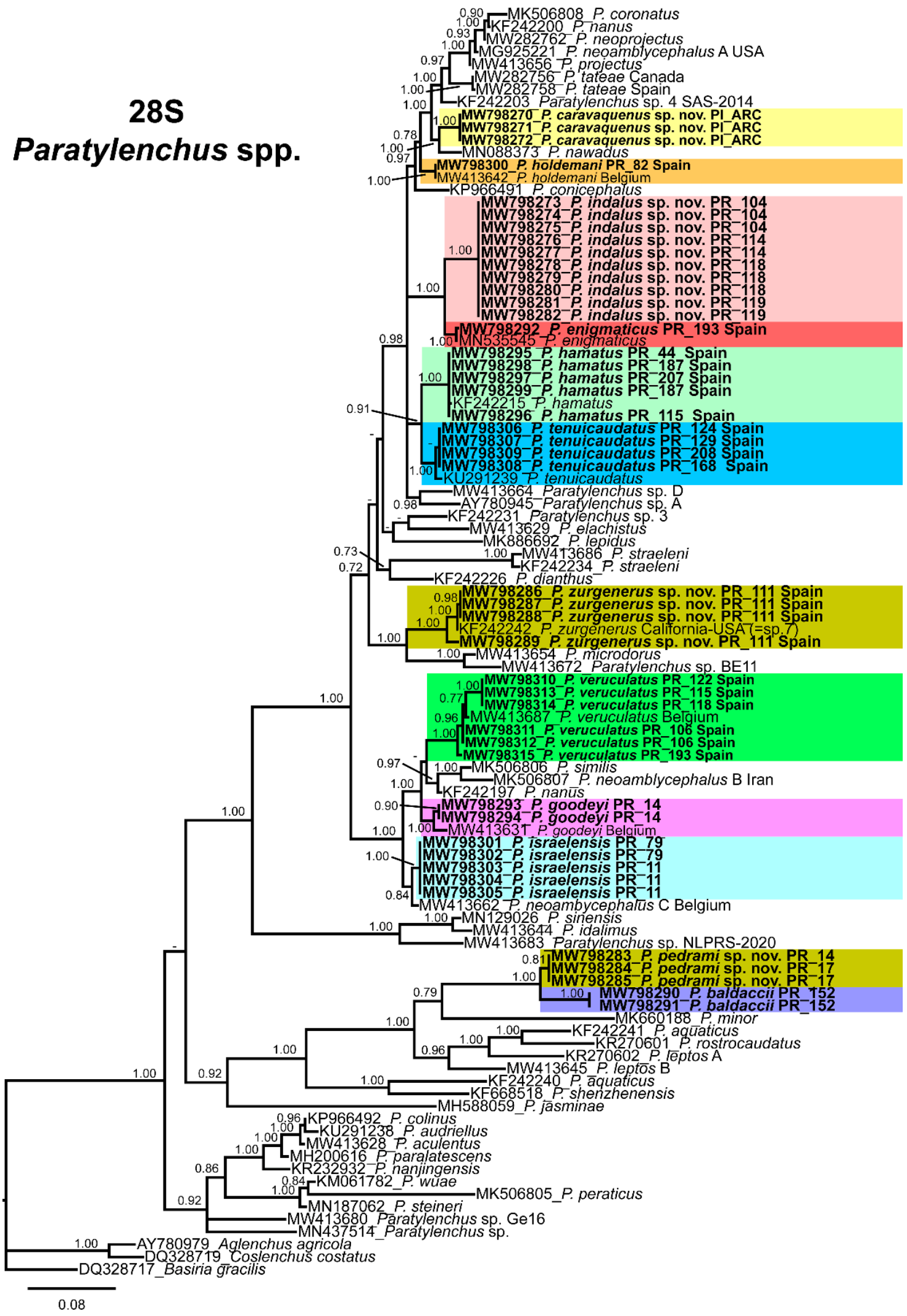

Figure 20. Phylogenetic relationships within the genus Paratylenchus. Bayesian 50\% majority rule consensus tree as inferred from D2-D3 expansion domains of the 28S rRNA sequence alignment under the general time-reversible model of sequence evolution with correction for invariable sites and a gamma-shaped distribution (GTR + I + G). Posterior probabilities of more than 0.70 are given for appropriate clades. Newly obtained sequences in this study are shown in bold. The scale bar indicates expected changes per site. 


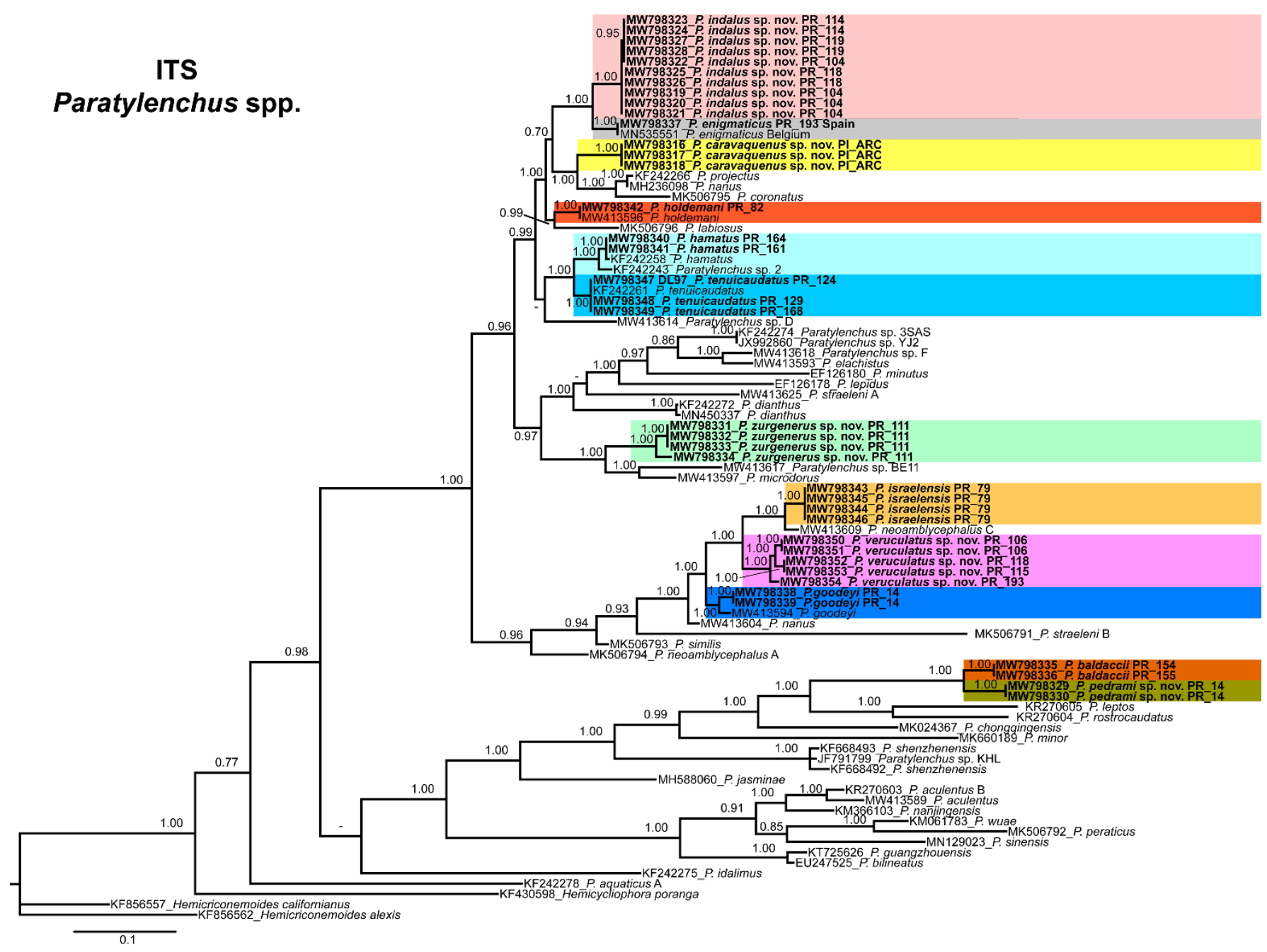

Figure 21. Phylogenetic relationships within the genus Paratylenchus. Bayesian 50\% majority rule consensus tree as inferred from ITS rRNA sequence alignment under the general time-reversible model of sequence evolution with correction for invariable sites and a gamma-shaped distribution (GTR $+\mathrm{I}+\mathrm{G})$. Posterior probabilities of more than 0.70 are given for appropriate clades. Newly obtained sequences in this study are shown in bold. The scale bar indicates expected changes per site.

Species identification with molecular markers deposited in GenBank was completely congruent with their phylogenetic position as it the case for P. hamatus, P. tenuicaudatus, P. holdemani, P. enigmaticus, P. veruculatus, and P. goodeyi (Figures 20-22). Species with new molecular information but already described in the literature include P. israelensis and P. baldaccii. Paratylenchus israelensis is closely related to P. neoamblycephalus C Belgium (MW413662) in a moderately supported clade $(\mathrm{PP}=0.84)$ in the $\mathrm{D} 2-\mathrm{D} 3$ domains of the $28 \mathrm{~S}$ rRNA, in a high-supported clade (PP = 1.00) in the ITS region (MW413609), and a low-supported clade ( $\mathrm{PP}<0.70)$ in the COI tree (MW421675-MW421681) (Figures 20-22). Paratylenchus baldaccii is closely related phylogenetically with P. pedrami sp. nov. as described before (Figures 20-22). 


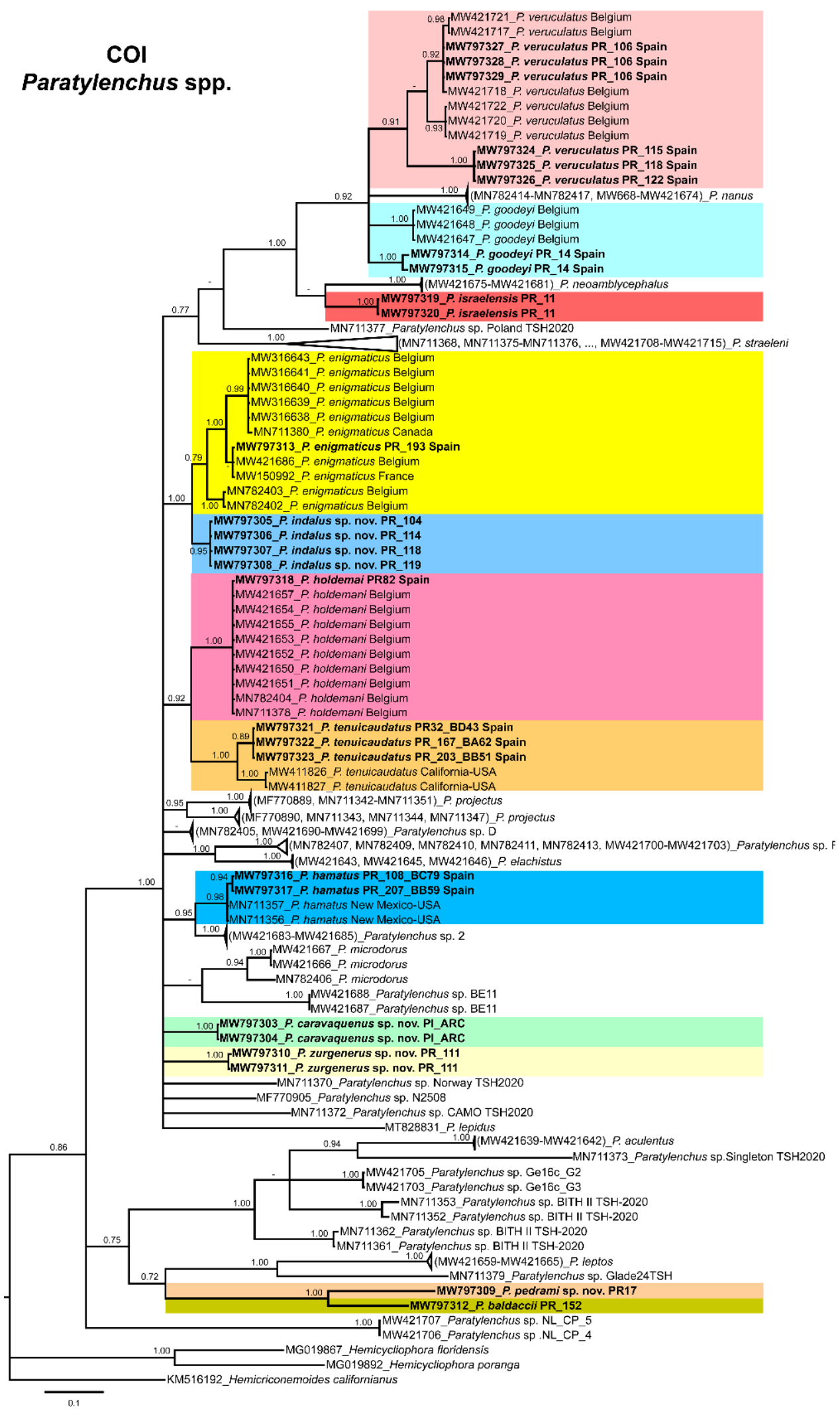

Figure 22. Phylogenetic relationships within the genus Paratylenchus. Bayesian 50\% majority rule consensus tree as inferred from COI sequence alignment under the general time-reversible model of sequence evolution with a gamma-shaped distribution $(G T R+G)$. Posterior probabilities of more than 0.70 are given for appropriate clades. Newly obtained sequences in this study are shown in bold. The scale bar indicates expected changes per site. 


\section{Discussion}

This research is the major study of pin nematodes of the genus Paratylenchus carried out in Spain, increasing their biodiversity, confirming the great cryptic morphology among several species, and expanding the number of species of this genus with molecular information for their unequivocal identification. Herein, we provide detailed morphological and molecular data on 27 studied populations from Spain and also discuss and confirm the existence of cryptic species as suggested in other plant-parasitic nematodes [85,86]. More specifically, four new species were described and additionally of them, five out of eight species identified are considered first reports in Spain (P. enigmaticus, P. hamatus, $P$. holdemani, $P$. israelensis, and P. veruculatus). This study also confirms that we have only found just a minor part of the species was already reported in Spain as P. baldaccii, P. goodeyi and $P$. tenuicaudatus $[44,48,52]$, indicating that the biodiversity of this group is far to be adequately explored in Spain and many of the data need to be revisited and complemented with molecular tools for their accurate identification by integrative taxonomy. However, our data in the present research were mainly based on agricultural systems and some of these species previously reported in Spain may not be coincident with our present species found mainly in Prunus plantations. Some of our data, even for new species, reinforces the idea that some of these species might be pathogenic in some periods of the year or feed with other plant hosts different from the main crop observed at the time of the soil sampling. This idea is reinforced because the majority of the species described here are in an arrested juvenile stage at the moment of sampling. Additionally, many ecological requirements of these nematodes are fairly deciphered (degree of soil humidity, temperature, etc.), and further studies are required for clarifying these aspects.

This study gave molecular markers for the first time for several Paratylenchus species for their accurate identification in an integrative taxonomic approach (including molecular and morphological traits). This is even more important where the presence of cryptic speciation is clearly detected in this genus (as for example, P. aquaticus, P. straeleni or $P$. hamatus $[23,24])$, with an excellent new example described in this study (i.e., $P$. indalus sp. nov., P. projectus, P. neoprojectus and P. enigmaticus). Several authors studied and give molecular markers in an integrative approach for this genus [21,23,24,26-29]. Singh et al. [24] gives a DNA-based species delimitation study using different calculation approaches and the markers most congruent between species separation by morphology and molecular identification was COI and D2-D3 region of 28S rRNA. These same authors recommend the use of multilocus approaches (D2-D3 region of 28S rRNA and COI) for a posterior double-check for contamination, sequencing errors of mitochondria-specific pitfalls [87]. Our data agrees with this result, but the phylogeny with COI marker was not completely congruent with the ribosomal molecular markers, and also some clades were low-supported. Phylogenetic analyses based on D2-D3, ITS, and partial COI gene using BI resulted in a consistent position for the newly described species of Paratylenchus species from Spain, and mostly agree with the clustering obtained by other authors [24,28]. Although the position of some species varies, probably due to the large number of species and additional molecular diversity included in this study. The molecular markers for this genus match with our identified species as mentioned in results, giving evidence that they could help in the identification process for the majority of our species. In our study, the majority of the species showed no or low intraspecific molecular variability for ribosomal and mitochondrial regions irrespective of the geographic origin of the population, with illustrative examples in P. indalus sp. nov., P. goodeyi or P. hamatus. The highest intraspecific variability for COI and ITS regions (95\%, 96\%, respectively) was found in P. veruculatus. Singh et al. [24] detected also important COI gene sequence variations within some species such as P. enigmaticus, P. microdorus and P. veruculatus, despite these sequences were originated from the same population. Usually, the most variable marker for plant-parasitic nematode species separation is COI, followed by ITS, D2-D3 region of 28S rRNA and partial $18 \mathrm{~S}$ rRNA $[85,88]$. However, in this study we found a different molecular variability among the different molecular markers between our four populations of $P$. indalus $\mathrm{sp}$. nov. 
and molecular markers for P. enigmaticus in Canada [21] and Belgium [24]. In this case, the variability is higher or similar for the D2-D3 region of 28S rRNA marker than for ITS region and COI ( $96 \%$ vs. $94-96 \%$ and $97 \%$ similarity for D2-D3 region of $28 \mathrm{~S}$ rRNA, ITS region and COI, respectively when compared both species). This point is reinforced because many sequences were obtained for $P$. indalus sp. nov. (ten D2-D3 of 28S rRNA, thirteen ITS, and four COI gene sequences) from the four different populations and all populations showed no intraspecific variation, except for the ITS sequences, where one variable position was found. This result is difficult to explain, and reinforces the idea that more than one marker is necessary for the molecularly species identification in this group of nematodes. In this case, the low molecular differences for COI marker needs to be explored in further studies with different mitochondrial markers or different primers for this gene.

Interestingly, the morphology in some complex species did not match the phylogenetic results, for example, the "P. hamatus complex" of species has some species closely related phylogenetically, but other far related such as P. pedrami sp. nov. and P. baldaccii, but these later related morphologically. Clear morphological characters did not match our phylogenies, for example the long stylet length $(>40 \mu \mathrm{m})$ for some species did not correspond with a unique clade (P. straeleni, $P$. goodeyi, $P$. idalimus.) as this character seems evolved independently several times in the different phylogenetic trees. Two clades of species seem to evolved from an exclusive long stylet ancestor, for example clade II ( $P$. idalimus, P. sinensis and others) or the subclade (P. colinus, P. audriellus, P. aculentus, P. paralatescens, P. nanjingensis and P. peraticus) in the D2-D3 region of $28 \mathrm{~S}$ rRNA tree, but not clearly supported in the other trees in this study or only partially in specific subclades (ITS region and COI). In this sense, the use of fresh material is essential to observe several characters in these nematodes as the presence/absence of stylet in male and juveniles, advulval flaps, lateral field incisures, and other characters difficult to visualize in the glycerin mounted specimens.

The distribution of many of these Paratylenchus species using an integrative approach showed that these species are more widely spread among continents that suspected. Cases as P. hamatus, P. tenuicaudatus, P. enigmaticus, ... ) shows us that these nematodes have a potential to be distributed on soil remains even with the absence of plants because of their resistant strategies not properly studied. As commented before, the majority of our samples are based on cultivated soils, but probably a higher diversity could be present in samples from wild environments.

\section{Conclusions}

This study describes and provides unequivocal molecular markers for the identification of different Paratylenchus species found in Spain. This is particularly important in this group of nematodes as only a few morphological characters can be used in their identification. As pointed in this and other studies, molecular markers could help in their identification, even when morphological characters might be variable and not overlapping ranges can be found. In summary, the present study confirmed the cryptic diversity of Paratylenchus species in Spain and comprises a good example of morphostatic speciation of pin nematodes in Spain. However, this genus is started recently to be studied by integrative taxonomy and increasing numbers of examples of cryptic diversity is expected to be found in the future.

Author Contributions: Conceptualization, J.E.P.-R., I.C.-C., C.C.-N., A.A.-Y. and P.C., methodology, I.C.-C., C.C.-N., P.C. and J.E.P.-R., software, I.C.-C., C.C.-N., A.A.-Y., P.C. and J.E.P.-R. analysis, I.C.-C., C.C.-N., A.A.-Y., P.C. and J.E.P.-R., resources, P.C. and J.E.P.-R., writing, I.C.-C., C.C.-N., A.A.-Y., P.C. and J.E.P.-R. All authors contributed to the final discussion data, and have read and agreed to the published version of the manuscript.

Funding: This research was supported by grant RTI2018-095925-A-100 from Ministerio de Ciencia, Innovación y Universidades, Spain.

Institutional Review Board Statement: Not applicable. 
Informed Consent Statement: Not applicable.

Data Availability Statement: The datasets generated during and/or analyzed during the current study are available from the corresponding author on reasonable request.

Acknowledgments: We would like to thank J. Martin Barbarroja and G. León Ropero (IAS-CSIC) for their excellent technical assistance in surveys and management of soil samples, as well as further anonymous reviewers and editors for their effort in reviewing the manuscript and helping improve this study. This research is part of the $\mathrm{PhD}$ project of the first author. The first author is a recipient of a contract from Ministry of Science and Innovation for Predoctoral Researchers in Spain, PRE2019090206. The third author is a recipient of the Humboldt Research Fellowship for Postdoctoral Researchers at the Helmholtz Centre for Environmental Research-UFZ, Leipzig, Germany.

Conflicts of Interest: The authors declare no conflict of interest. The funders had no role in the design of the study; in the collection, analyses, or interpretation of data; in the writing of the manuscript, or in the decision to publish the results.

\section{References}

1. Micoletzky, H. Die Freilebenden Erd-Nematoden; Archiv für Naturgeschichte: Berlin, Germany, 1922.

2. Ghaderi, R.; Geraert, E.; Karegar, A. The Tylenchulidae of the World, Identification of the Family Tylenchulidae (Nematoda: Tylenchida); Academia Press: Ghent, Belgium, 2016.

3. Ghaderi, R. The damage potential of pin nematodes, Paratylenchus Micoletzky, 1922 sensu lato spp. (Nematoda: Tylenchulidae). J. Crop Prot. 2019, 8, 243-257.

4. Bongers, T.; Ferris, H. Nematode community structure as a bioindicator in environmental monitoring. Trends Ecol. Evol. 1999, 14, 224-228. [CrossRef]

5. Schmidt, J.H.; Seeger, J.N.; Von Grafenstein, K.; Wintzer, J.; Finckh, M.R.; Hallmann, J. Population dynamics and host range of Paratylenchus bukowinensis. Nematology 2020, 22, 257-267. [CrossRef]

6. Yeates, G.W.; Lee, W.G. Burning in a New Zealand snow-tussock grassland: Effects on vegetation and soil fauna. N. Z. J. Ecol. 1997, 21, 73-79.

7. Gaur, H.S. Dissemination and mode of survival of nematodes in dust storms. Indian J. Nematol. 1988, 18, 94-98.

8. Brzeski, M.W. Paratylenchus Bukowinensis. CIH Descriptions of Plantparasitic Nematodes. Set 6, N 79; Commonwealth Agricultural Bureau: Farnham Royal, UK, 1976.

9. Brzeski, M.W. Pathogenicity of Paratylenchus bukowinensis Micol. on celery. Rocz. Nauk Rol. Ser. E 1975, 5, 23-29.

10. Pennacchio, F.; D’Errico, F.P.; Tremblay, E. Spatial distribution pattern and sequential sampling plan for Paratylenchus dianthus. Nematol. Medit. 1985, 13, 137-146.

11. Allen, M.W.; Jensen, H.J. Cacopaurus epacris, new species (Nematoda: Criconematidae), a nematode parasite of California black walnut roots. Proc. Helm. Soc. Wash. 1950, 17, 10-14.

12. Thorne, G.; Allen, M.W. Paratylenchus hamatus n.sp, and Xiphinema index n.sp., two nematodes associated with fig roots, with a note on Paratylenchus anceps Cobb. Proc. Helm. Soc. Wash. 1950, 17, $27-35$.

13. French, A.; Lownsbery, B.; Ayoub, S.; Weiner, A.; El-Gholl, N. Pythiaceous fungi and plant-parasitic nematodes in California pear orchards: II. Incidence and distribution of parasitic nematodes in orchard soils. Hilgardia 1964, 35, 603-610. [CrossRef]

14. Philis, J. Controlling parasitic nematodes in an established vineyard in Cyprus. Nematol. Medit. 2003, $31,61-63$.

15. Andrássy, I. Paratylenchus microdorus. CIH Descriptions of Plant-Parasitic Nematodes. Set 8, No. 107; Commonwealth Agricultural Bureau: Farnham Royal, UK, 1985.

16. Odihirin, R.A.; Jenkins, W.R. Host-parasite relationship of impatiens balsamina and certain nematodes. Phytopathology 1965, $55,736-766$

17. Braun, A.L.; Lownsbery, B.F. The pin nematode, Paratylenchus neoamblycephalus, on Myrobalan plum and other hosts. J. Nematol. 1975, 7, 336-343.

18. Webster, G.R.; Orchard, W.R.; Hawn, E.J. Paratylenchus projectus in alfalfa fields of central and northern Alberta. Can. Plant Dis. Surv. 1972, 52, 75-76.

19. Smolik, J.D. Effects of Paratylenchus projectus on growth of sunflower. Plant Dis. 1987, 71, 975-976. [CrossRef]

20. Wang, K.; Li, Y.; Xie, H.; Wu, W.-J.; Xu, C.-L. Pin nematode slow decline of Anthurium andraeanum, a new disease caused by the pin nematode Paratylenchus shenzhenensis. Plant Dis. 2016, 100, 940-945. [CrossRef]

21. Munawar, M.; Yevtushenko, D.P.; Palomares-Rius, J.E.; Castillo, P. Species diversity of pin nematodes (Paratylenchus spp.) from potato growing regions of southern Alberta, Canada. Plants 2021, 10, 188. [CrossRef]

22. Claerbout, J.; Vandevelde, I.; Venneman, S.; Kigozi, A.; Sutter, N.d.; Neukermans, J.; Bleyaert, P.; Bert, W.; Höfte, M.; Viaene, N. A thorough study of a Paratylenchus sp. in glasshouse-grown lettuce: Characterisation, population dynamics, host plants and damage threshold as keys to its integrated management. Ann. Appl. Biol. 2021, 178, 62-79. [CrossRef] 
23. Van den Berg, E.; Tiedt, L.R.; Subbotin, S.A. Morphological and molecular characterisation of several Paratylenchus Micoletzky, 1922 (Tylenchida: Paratylenchidae) species from South Africa and USA, together with some taxonomic notes. Nematology 2014, 16, 323-358. [CrossRef]

24. Singh, R.; Karssen, G.; Coureur, M.; Subbotin, S.; Bert, W. Integrative taxonomy and molecular phylogeny of the plant-parasitic nematode genus Paratylenchus (Nematoda: Paratylenchinae): Linking species with molecular barcodes. Plants 2021, 10, 408. [CrossRef] [PubMed]

25. Fisher, J.M. Studies on Paratylenchus nanus I. effects of variation in environment on several morphometric characters of adults. Nematologica 1965, 11, 269-279. [CrossRef]

26. Zhuo, K.; Liu, X.; Tao, Y.; Wang, H.; Lin, B.; Liao, J. Morphological and molecular characterisation of three species of Paratylenchus Micoletzky, 1922 (Tylenchida: Paratylenchidae) from China, with a first description of the male P. rostrocaudatus. Nematology 2018, 20, 837-850. [CrossRef]

27. Mirbabaei, H.; Eskandari, A.; Ghaderi, R.; Karegar, A. On the synonymy of Trophotylenchulus asoensis and T. okamotoi with T. arenarius, and intra-generic structure of Paratylenchus (Nematoda: Tylenchulidae). J. Nematol. 2019, 51, 1-22. [CrossRef]

28. Munawar, M.; Miao, W.; Castillo, P.; Zheng, J.-W. A new pin nematode, Paratylenchus sinensis n. sp. (Nematoda: Paratylenchinae) in the rhizosphere of white mulberry from Zhejiang Province, China. Eur. J. Plant Pathol. 2020, 156, 1023-1029. [CrossRef]

29. Subbotin, S.A.; Yan, G.; Kantor, M.; Handoo, Z. On the molecular identity of Paratylenchus nanus Cobb, 1923 (Nematoda: Tylenchida). J. Nematol. 2020, 52, 1-7. [CrossRef]

30. Sturhan, D.; Geraert, E. Phasmids in Tylenchulidae (Tylenchida: Criconematoidea). Nematology 2005, 7, 249-252. [CrossRef]

31. Subbotin, S.A.; Vovlas, N.; Crozzoli, R.; Sturhan, D.; Lamberti, F.; Moens, M.; Baldwin, J.G. Phylogeny of Criconematina Siddiqi, 1980 (Nematoda: Tylenchida) based on morphology and D2-D3 expansion segments of the 28S-rRNA gene sequences with application of a secondary structure model. Nematology 2005, 7, 927-944. [CrossRef]

32. Subbotin, S.A.; Sturhan, D.; Chizhov, V.N.; Vovlas, N.; Baldwin, J.G. Phylogenetic analysis of Tylenchida Thorne, 1949 as inferred from D2 and D3 expansion fragments of the 28S rRNA gene sequences. Nematology 2006, 8, 455-474. [CrossRef]

33. Brzeski, M.W. Nematodes of Tylenchina in Poland and Temperate Europe; Muzeum i Instytut Zoologii Polska Akademia Nauk: Warsaw, Poland, 1998.

34. Nguyen, C.N.; Baldwin, J.G.; Choi, Y.E. New records of Paratylenchus Micoletzky, 1922 (Nematoda: Paratylenchinae) from Viet Nam with description of Paratylenchus lapcaiensis sp. n. J. Nematode Morphol. Syst. 2004, 7, 51-75.

35. Decraemer, W.; Hunt, D.J. Structure and classification. Plant Nematol. 2006, 3-32. [CrossRef]

36. Ghaderi, R.; Kashi, L.; Karegar, A. Contribution to the study of the genus Paratylenchus Micoletzky, 1922 sensu lato (Nematoda: Tylenchulidae). Zootaxa 2014, 3841, 151-187. [CrossRef]

37. Hesar, A.M.; Karegar, A.; Ghaderi, R. Phylogenetic relationships of Cacopaurus pestis Thorne, 1943 within representatives of the Tylenchulidae Skarbilovich, 1947 as inferred from ITS and D2-D3 expansion segments of 28S-rRNA sequences. Nematology 2019, 21, 971-994. [CrossRef]

38. Thorne, G. Cacopaurus pestis, n. g., n. spec. (Nematoda: Criconematinae), a destructive parasite of the walnut, Juglans regia Linn. Proc. Helm. Soc. Wash. 1943, 10, 78-83.

39. Goodey, T. Soil and Freshwater Nematodes, 2nd ed.; Goodey, J.B., Ed.; Methuen: London, UK, $1963 ;$ p. 544.

40. Siddiqi, M.R. Tylenchida: Parasites of Plants and Insects, 2nd ed.; CABI Publishing: Wallingford, UK, 2000.

41. Andrássy, I. Free-Living Nematodes of Hungary, II (Nematoda, Errantia); Hungarian Natural History Museum: Budapest, Hungary, 2007.

42. Brzeski, M.; Hanel, L.; Nico, A.; Castillo, P. Paratylenchinae: Redescription of Paratylenchus arculatus Luc \& de Guiran, 1962, a new senior synonym of P. nainianus Edward \& Misra, 1963 (Nematoda: Tylenchulidae). Nematology 1999, 1, 375-380. [CrossRef]

43. Nico, A.I.; Rapoport, H.F.; Jiménez-Díaz, R.M.; Castillo, P. Incidence and population density of plant-parasitic nematodes associated with olive planting stocks at nurseries in southern Spain. Plant Dis. 2002, 86, 1075-1079. [CrossRef] [PubMed]

44. Peña-Santiago, R. Plant-parasitic nematodes associated with olive (Olea europea L.) in the province of Jaén, Spain. Rev. Nématol. 1990, 13, 113-115.

45. Gomez-Barcina, A.; Castillo, P.; Pais, M.A.G. Four species of the genus Paratylenchus Micoletzky from Southeasthern Spain. Nematol. Medit. 1990, 18, 169-177.

46. Talavera, M.; Navas, A. Incidence of plant-parasitic nematodes in natural and semi-natural mountain grassland and the host status of some common grass species. Nematology 2002, 4, 541-552. [CrossRef]

47. Archidona-Yuste, A.; Wiegand, T.; Castillo, P.; Navas-Cortés, J.A. Dataset on the diversity of plant-parasitic nematodes in cultivated olive trees in southern Spain. Data Brief 2019, 27, 104658. [CrossRef]

48. Castillo, P.; González-País, M.A.; Gómez-Barcina, A. El género Gracilacus Raski, 1962 en España (Paratylenchinae: Tylenchida). Rev. Ibér. Parasitol. 1989, 49, 321-328.

49. Talavera, M.; Tobar Jimenez, A. Plant parasitic nematodes from unirrigated fields in Alhama, southeastern Spain. Nematol. Medit. 1997, 25, 73-81.

50. Castillo, P.; Gómez-Barcina, A. Some species of Tylenchida from natural habitats in southeastern Spain. Nematol. Medit. 1988, 16, 75-86.

51. Peña Santiago, R.; Geraert, E. New data on Aorolaimus perscitus (Doucet, 1980) and Gracilacus teres Raski, 1976 (Nematoda: Tylenchida) associated with olive (Olea europea L.) in the province of Jaén, Spain. Nematologica 1990, 36, 408-416. [CrossRef] 
52. Hernandez, M.; Mateo, M.D.; Jordana, R. Estudio comparativo entre grupos tróficos de Nematodos del suelo de cinco bosques de Navarra (tres naturales y dos de repoblación). Actas II Congr. Mund. Vasco. Sec. Biol. Ambient. 1988, 2, $323-335$.

53. Coolen, W.A. Methods for extraction of Meloidogyne spp. and other nematodes from roots and soil. In Root-Knot Nematodes (Meloidogyne Species). Systematics, Biology and Control; Lamberti, F., Taylor, C.E., Eds.; Academic Press: New York, NY, USA, 1979; pp. 317-329.

54. Seinhorst, J.W. Killing nematodes for taxonomic study with hot F.A. 4:1. Nematologica 12, 178. Nematologica 1966, 12, 178. [CrossRef]

55. De Grisse, A.T. Redescription ou modifications de quelques techniques utilisées dans l'étude de nématodes phytoparasitaires. Meded. Rijksfac. Landbouwwet. Gent 1969, 34, 315-359.

56. Hunt, D.J.; Palomares-Rius, J.E. General morphology and morphometries of plant-parasitic nematodes. In Practical Plant Nematology; Biblioteca Basica de Agricultura: Montecillo, Mexico, 2012; pp. 25-64.

57. Palomares-Rius, J.E.; Clavero-Camacho, I.; Archidona-Yuste, A.; Cantalapiedra-Navarrete, C.; León-Ropero, G.; Braun Miyara, S.; Karssen, G.; Castillo, P. Global distribution of the reniform nematode genus Rotylenchulus with the synonymy of Rotylenchulus macrosoma with Rotylenchulus borealis. Plants 2021, 10, 7. [CrossRef]

58. De Ley, P.; Felix, M.A.; Frisse, L.; Nadler, S.; Sternberg, P.; Thomas, W.K. Molecular and morphological characterisation of two reproductively isolated species with mirror-image anatomy (Nematoda: Cephalobidae). Nematology 1999, 1, 591-612. [CrossRef]

59. Subbotin, S.A.; Vierstraete, A.; De Ley, P.; Rowe, J.; Waeyenberge, L.; Moens, M.; Vanfleteren, J.R. Phylogenetic relationships within the cyst-forming nematodes (Nematoda, Heteroderidae) based on analysis of sequences from the ITS regions of ribosomal DNA. Mol. Phylogenet. Evol. 2001, 21, 1-16. [CrossRef]

60. Bowles, J.; Blair, D.; McManus, D.P. Genetic variants within the genus Echinococcus identified by mitochondrial DNA sequencing. Mol. Biochem. Parasitol. 1992, 54, 165-173. [CrossRef]

61. Altschul, S.F.; Gish, W.; Miller, W.; Myers, E.W.; Lipman, D.J. Basic local alignment search tool. J. Mol. Biol. 1990, 215, 403-410. [CrossRef]

62. Katoh, K.; Rozewicki, J.; Yamada, K.D. MAFFT online service: Multiple sequence alignment, interactive sequence choice and visualization. Brief. Bioinform. 2019, 20, 1160-1166. [CrossRef] [PubMed]

63. Hall, T.A. BioEdit: A user-friendlybiological sequence alignment editor and analysis program for Windows 95/98/NT. Nucleic Acids Symp. Ser. 1999, 41, 95-98.

64. Castresana, J. Selection of conserved blocks from multiple alignments for their use in phylogenetic analysis. Mol. Biol. Evol. 2000, 17, 540-552. [CrossRef] [PubMed]

65. Ronquist, F.; Huelsenbeck, J.P. MrBayes 3: Bayesian phylogenetic inference under mixed models. Bioinformatics 2003, $19,1572-1574$. [CrossRef] [PubMed]

66. Darriba, D.; Taboada, G.L.; Doallo, R.; Posada, D. jModelTest 2: More models, new heuristics and parallel computing. Nat. Methods 2012, 9, 772. [CrossRef]

67. Rambaut, A. FigTree v1.4.2, A Graphical Viewer of Phylogenetic Trees. Available online: http://tree.bio.ed.ac.uk/software/ figtree/ (accessed on 27 March 2021).

68. Oostenbrink, M. A note on Paratylenchus in the Netherlands with the description of P. goodeyi n. sp. (Nematoda, Criconematidae). Tijdschr. Plantenziekten 1953, 59, 207-216. [CrossRef]

69. Geraert, E. The Genus Paratylenchus. Nematologica 1965, 11, 301-334. [CrossRef]

70. Sturhan, D. Plant-parasitic nematodes in Germany-An annotated checklist. Soil Org. 2014, 83, 22.

71. Izatullaeva, R.I. Nematodes of the Flowering Plants of Kazakhstan; Abstract Kandidat's Dissertation: Alma-Ata, Kazakhstan, 1967; pp. 1-18.

72. Lisetskaya, L.F. Nematode fauna of ethereal oil plants of Moldavia. Mater. Nats. Konf. Po Parazitol. Sofia 1968, $13,47-48$.

73. Solov'eva, G.I. Parasitic Nematodes of Woody and Herbaceous Plants. A Review of the Genus Paratylenchus Micoletzky, 1922 (Nematoda: Criconematidae); Amerind Publishing Co.: New Delhi, India, 1975; p. 134.

74. Szczygiel, A. Plant parasitic nematodes associated with strawberry plants in Poland. Zesz. Probl. Postepów Nauk Rol. 1974, 154, 1-132.

75. Brzeski, M.W. Paratylenchinae: Morphology of Some Known Species and Descriptions of Gracilacus Bilineata Sp. N. and G. Vera Sp. N. (Nematoda: Tylenchulidae). Nematologica 1995, 41, 535-565. [CrossRef]

76. Háněl, L.; Čerevková, A. Diversity of soil nematodes in meadows of the White Carpathians. Helminthologia 2006, 43, 109-116. [CrossRef]

77. Raski, D.J. Revision of the genus Paratylenchus Micoletzky, 1922 and descriptions of new species. Part II of three parts. J. Nematol. 1975, 7, 274-295.

78. Powers, T.O.; Harris, T.S.; Higgins, R.S.; Mullin, P.G.; Powers, K.S. Nematode biodiversity assessments need vouchered databases: A BOLD reference library for plant-parasitic nematodes in the superfamily Criconematoidea. Genome 2020, 64, 232-241. [CrossRef] [PubMed]

79. Raski, D.J. Paratylenchoides gen. n. and two new Species (Nematoda: Paratyleiichidae). Proc. Helm. Soc. Wash. 1973, 40, $230-233$.

80. Myers, R.F. Plant Parasitic Nematodes in New Jersey. J. Nematol. 1986, 18, 272-274. [PubMed] 
81. Esmaeili, M.; Heydari, R.; Castillo, P.; Bidhendi, M.Z.; Palomares-Rius, J.E. Molecular characterisation of two known species of Paratylenchus Micoletzky, 1922 from Iran with notes on the validity of Paratylenchus audriellus Brown, 1959. Nematology 2016, 18, 591-604. [CrossRef]

82. Wu, L.Y. Paratylenchus veruculatus n. sp. (Criconematidae: Nematoda) from Scotland. Can. J. Zool. 1962, 40, 773-775. [CrossRef]

83. Raski, D.J. Revision of the genus Paratylenchus Micoletzky, 1922 and descriptions of new species. Part I of three parts. J. Nematol. $1975,7,15-34$.

84. Ghaderi, R.; Karegar, A. Some species of Paratylenchus (Nematoda: Tylenchulidae) from Iran. Iran. J. Plant Pathol. 2013, 49, 137-156.

85. Palomares-Rius, J.E.; Cantalapiedra-Navarrete, C.; Castillo, P. Cryptic species in plant-parasitic nematodes. Nematology 2014, 16, 1105-1118. [CrossRef]

86. Archidona, Y.; Cai, R.; Cantalapiedra-Navarrete, C.; Carreira, J.A.; Rey, A.; Viñegla, B.; Liébanas, G.; Palomares-Rius, J.E.; Castillo, P. Morphostatic Speciation within the Dagger Nematode Xiphinema hispanum-Complex Species (Nematoda: Longidoridae). Plants 2020, 9, 1649. [CrossRef]

87. Jörger, K.M.; Norenburg, J.L.; Wilson, N.G.; Schrödl, M. Barcoding against a paradox? Combined molecular species delineations reveal multiple cryptic lineages in elusive meiofaunal sea slugs. BMC Evol. Biol. 2012, 12, 245. [CrossRef] [PubMed]

88. Palomares-Rius, J.E.; Cantalapiedra-Navarrete, C.; Archidona-Yuste, A.; Subbotin, S.A.; Castillo, P. The utility of mtDNA and rDNA for barcoding and phylogeny of plant-parasitic nematodes from Longidoridae (Nematoda, Enoplea). Sci. Rep. 2017, 7, 10905. [CrossRef] [PubMed] 